Sistemas dinâmicos discretos: estabilidade, comportamento assintótico e sincronização 



\title{
Sistemas dinâmicos discretos: estabilidade, comportamento assintótico e sincronização ${ }^{1}$
}

\author{
Wescley Bonomo
}

Orientador: Prof. Dr. Hildebrando Munhoz Rodrigues

Dissertação apresentada ao Instituto de Ciências Matemáticas e de Computação da Universidade de São Paulo, como parte dos requisitos para obtenção do título de Mestre em Ciências - Área: Matemática.

\section{USP - São Carlos}

Abril/2008

\footnotetext{
${ }^{1}$ Este trabalho teve suporte financeiro da CAPES
} 

"A Matemática é o mais maravilhoso instrumento criado pelo gênio do homem para a descoberta da verdade."

(Laisant)

"Felizes aqueles que se divertem com problemas que educam a alma e elevam o espírito."

(Fenelon)

"O valor de nossas expectativas sempre significa algo entre o melhor que podemos esperar e o pior que podemos temer." (Jacob Bernoulli)

"Não existe oceano maior do que a determinação humana." (Lars Grael)

"Aqui, no entanto, nós não olhamos para trás por muito tempo.

Nós continuamos seguindo em frente, abrindo novas portas e fazendo coisas novas, porque somos curiosos ... e a curiosidade continua nos conduzindo por novos caminhos."

(Walt Disney)

Ouvi, que não vereis com vãs façanhas, Fantásticas, fingidas, mentirosas, Louvar os vossos, como nas estranhas Musas, de engrandecer-se desejosas: As verdadeiras vossas são tamanhas, Que excedem as sonhadas, fabulosas; Que excedem Rodamonte, e o vão Rugeiro, E Orlando, inda que fora verdadeiro, (Luiz Vaz de Camões em Os Lusíadas) 



\section{Ofereço}

Aos meus pais

Anna e Matheus

Ao meu irmão

Wanderson

A minha avó

Maria Bonomo Vinhati

A memória dos meus avós

Nilo Vinhati;

Amélia Anastácio Bonomo;

Matheus Bonomo. 



\section{Agradecimentos}

Inicialmente, agradesço aos meus pais Matheus e Anna, por terem me dado a vida, e nos quais me espelhei para criar meus valores e me tornar a pessoa que hoje sou, por me darem grande apoio moral, me encorajando em todos os momentos da minha vida. Ao meu irmão Wanderson e também aos meus primos Charles, Verusca e Charla, ora irmãos mais velhos e sempre amigos, pelas brincadeiras que fizemos juntos e também pelo zelo que deram a minha educação, minha eterna gratidão!

Ao professor Hildebrando pelo tema proposto e pela forma como orientou esse trabalho, permitindo com que eu trabalhasse com liberdade.

Aos meus professores do DM-UFES; em especial ao Alancardek e a Liane pelo incentivo dado para que eu cursasse o mestrado; aos meus orientadores do PET: Jamil Ferreira e José A. da Rocha Pinto, e também aos meus professores de mestrado, com os quais obtive uma formaçao mais sólida, a qual certamente vai além das salas de aula do ICMC-USP.

Ao professor Daniel Vendruscolo, por ter me ajudado a superar obstáculos encontrados pelo caminho, durante o mestrado.

A minha turma de mestrado: Eduardo, Giuliano, J. Claudinei, Juliano, Lucas, Marcos, Thais, Thiago Castilho, Thiago Catalan e Yuri; ao pessoal da minha sala, com os quais tive a oportunidade de dividir a maior parte dos meus dias de trabalho; aos amigos do ICMC pelo companheirismo e pela amizade, em especial a Daniela. Aos meus eternos amigos, àqueles que me acompanharam e me ajudaram de perto e aqueles que torceram por mim de longe.

Ao pessoal do apartamento 406 do condomínio onde morei parte do tempo que fiz esse mestrado: Ronaldinho e Flank, e aos demais amigos do prédio; Zenilde, Raulina, Neli, Nélia, Pedro, Jane, Leu, e o zelador Marcos.

A CAPES pelo suporte financeiro concedido durante o mestrado, e pela bolsa concedida nos estudos do PET, durante a graduação.

Enfim, a todos aqueles que de alguma forma contribuíram para a realização desse trabalho.

Obrigado! 



\section{Resumo}

Este trabalho é em parte baseado no livro The Stability and Control of Discrete Processes de Joseph P. LaSalle. Nós estudamos equações como $x(n+1)=T(x(n))$, onde $T: \mathbb{R}^{m} \rightarrow \mathbb{R}^{m}$ é uma aplicação contínua, com o sistema dinâmico associado $\Pi(n, x):=T^{n}(x)$.

Nós fornecemos condições suficientes para a estabilidade de equilíbrios usando o método direto de Liapunov. Também consideramos sistemas discretos da forma $x(n+1)=T(n, x(n), \lambda)$ dependendo de uma parâmetro $\lambda$ e apresentamos resultados obtendo estimativas de atratores.

Finalmente, nós apresentamos algumas simulações de sistemas acoplados como uma aplicação em sistemas de comunicação. 



\section{Abstract}

This work is in part based on the book The Stability and Control of Discrete Processes of Joseph P. LaSalle. We studing equations as $x(n+1)=T(x(n))$, where $T: \mathbb{R}^{m} \rightarrow \mathbb{R}^{m}$ is continuous transformation, with the associated dynamic system $\Pi(n, x):=T^{n}(x)$.

We provide suddicient conditions for stability of equilibria, using Liapunov direct method. We also consider nonautonomous discrete systems of the form $x(n+1)=T(n, x(n), \lambda)$ depending on the parameter $\lambda$ and present results obtaining uniform estimatives of attractors.

We finally we present some simulations on synchronization of coupled systems as an application on communication systems. 



\section{Sumário}

Introdução

1 Preliminares 5

1.1 Equações de diferenças . . . . . . . . . . . . . . . . . . . . 5

1.2 Sistemas dinâmicos discretos . . . . . . . . . . . . . . . . . 6

1.3 Invariância . . . . . . . . . . . . . . . . . . 6

1.4 Conjuntos limite . . . . . . . . . . . . . . . . . . . 12

2 Estabilidade e instabilidade $\quad 19$

2.1 Método direto de Liapunov . . . . . . . . . . . . . . . . . . . . . 19

2.2 Versão estendida do princípio de invariância . . . . . . . . . . . . . . . . . . . 24

2.3 Simulações e exemplos . . . . . . . . . . . . . . . . . . . . . 28

3 Sistemas lineares $\quad 43$

3.1 Sistemas lineares de equações de diferenças homogêneas de primeira ordem . . 43

3.2 Forma canônica de Jordan . . . . . . . . . . . . . . . . . . . . 44

3.3 Critério de Schur-Cohn . . . . . . . . . . . . . . . . . . . . . . 48

3.4 Um funcional de Liapunov para $X^{\prime}=A X \ldots \ldots$. . . . . . . . . . 49

3.5 Estabilidade por aproximação linear . . . . . . . . . . . . . . . . . 52

3.6 Equações de diferenças lineares de ordem $m \ldots \ldots$. . . . . . . . . . . 53

3.7 Soluções de uma equação de m-ésima ordem . . . . . . . . . . . . . . . . . 54

3.8 Fórmula da variação das constantes . . . . . . . . . . . . . . . 55

3.9 Matrizes companheiras . . . . . . . . . . . . . . 56

4 Aplicações a sincronização $\quad \mathbf{5 7}$

4.1 Aplicações ao sistema de Lorenz discreto . . . . . . . . . . . . . . . . . . 57

$\begin{array}{ll}\text { Bibliografia } & 63\end{array}$ 



\section{Introdução}

Este trabalho está em parte baseado no livro The Stability and Control of Discrete Processes de Joseph P LaSalle, (veja [18]) e no preprint Uniform Dissipativeness and Robust Synchronization of Parametrized Discrete Systems: location of the Atractor (Veja RODRIGUES, WU e GABRIEL, [31]).

Uma igualdade da forma $F(n, x(n), x(n+1), x(n+2), \ldots, x(n+m))=0$, onde a variável $x$ é uma seqüência com valores em $\mathbb{R}^{m}$, chama-se equação de diferenças finitas. Resolver uma tal equação é encontrar uma sequiência $x: \mathbb{N} \rightarrow \mathbb{R}^{m}$ que a satisfaz para todo $n \in \mathbb{N}$.

Em muitas aplicações matemáticas como economia, o tempo é discreto, isto é, as grandezas são medidas em instantes isolados (de hora em hora, a cada segundo, etc.), formando uma sequiência que descreve o sistema. Neste caso as equações diferenciais não são adequadas para exprimir o a evolução do fenômeno, sendo substituídas pelas equações de diferenças finitas.

Mesmo equações diferenciais $\frac{d x}{d t}=f(x)$ podem ser convertidas em equações de diferenças através do método de Euler: $x(n+1)=x(n)+h f(x(n))$, que é um método iterativo de primeira ordem utilizado por exemplo na integração numérica e pelos microcomputadores.

Equações de diferenças finitas $x(n+1)=T(x(n))$ têm uma estrutura natural de sistema semidinâmico discreto (em $\left.\mathbb{R}^{m}\right)$ e a formulação matemática deste é baseada na lei de evolução $\Pi: \mathbb{N} \times \mathbb{R}^{m} \rightarrow \mathbb{R}^{m}$ dada por $\Pi(n, x):=T^{n}(x)$, onde o operador $T: \mathbb{R}^{m} \rightarrow \mathbb{R}^{m}$ por todo este trabalho será uma aplicação contínua. Eventualmente, estudaremos também sistemas do tipo $\Pi: \mathbb{N} \times \Lambda \times \mathbb{R}^{m} \rightarrow \mathbb{R}^{m}, \Pi(n, \lambda, x)=T_{\lambda}^{n}(x)$, o qual pode ser interpretado como uma família de sistemas semidinâmicos discretos. No capítulo 02 apresentaremos o Princípio da dissipação uniforme, provado por GABRIEL (veja [8], página 37 ou [31]). Esse resultado é assim chamado pois ele fornece uma estimativa uniforme (com relação ao parâmetro $\lambda \in \Lambda$ ) do atrator de $\Pi$ : $\mathbb{N} \times \Lambda \times \mathbb{R}^{m} \rightarrow \mathbb{R}^{m}$, como veremos.

A teoria dos sistemas dinâmicos interessa-se em descrever como as órbitas de $\Pi$, que são seqüências $x: \mathbb{N} \times \mathbb{R}^{m} \rightarrow \mathbb{R}^{m}$ se comportam e o que acontece com as mesmas quando $n$ vai para infinito. Este trabalho procurou estudar alguns aspectos qualitativos da teoria dos sistemas semidinâmicos discretos, com ênfase para a estabilidade, comportamento assintótico 
e controlabilidade dos mesmos.

No capítulo 01 estudamos os sistemas do tipo $x(n+1)=T(x(n))$ junto com a sua dinâmica associada $\Pi(n, x):=T^{n}(x)$, e algumas noções básicas da teoria dos sistemas dinâmicos, como os conceitos de invariância, órbitas, atratores, pontos fixos (ou estados de equilíbrio) e periódicos e conjuntos limites.

O Capítulo 02 preocupa-se em estudar a estabilidade dos estados de equilíbrio de $x(n+1)=$ $T(x(n))$. Uma das técnicas fundamentais utilizadas é o método direto de Liapunov, devido a Aleksandr Mikhalovich Liapunov (06/06/1857 - 03/11/1918).

Esta técnica fornece uma condição suficiente para a estabilidade de um ponto de equilíbrio. Para isso necessitamos da existência de uma função do tipo Liapunov $V: \mathbb{R}^{m} \rightarrow \mathbb{R}$; definimos a derivada discreta de $V$ (ao longo das soluções) como $\dot{V}(x):=V(T(x))-V(x)$. Os teoremas de estabilidade de Liapunov procuram obter informações qualitativas sobre o comportamento assintótico das soluções do sistema acima, a partir de propriedades das funções $V$ e $\dot{V}$, não sendo necessário o cálculo analítico das soluções.

Joseph. P. LaSalle estabeleceu uma relação entre funções de Liapunov e conjuntos $\omega$-limites de Birkhoff, desenvolvendo o principio da invariância de LaSalle.

Na prática trabalhamos com funcões de Liapunov generalizadas, a qual pode assumir valores negativos num conjunto limitado, e com isso podemos trabalhar com uma classe maior de problemas. Estudaremos sistemas do tipo $\Pi: \mathbb{N} \times \Lambda \times \mathbb{R}^{m} \rightarrow \mathbb{R}^{m}, \Pi(n, \lambda, x)=T_{\lambda}^{n}(x)$. No capítulo 02 apresentaremos o Princípio da dissipação uniforme, assim chamado pois fornece uma estimativa uniforme do atrator do sistema.

Resultados obtidos na dissertação de mestrado de Luiz Roberto Almeida Gabriel Filho, "Comportamento assintótico de sistemas não lineares discretos", orientada pelo professor Hildebrando Munhoz Rodrigues, (veja [8]) na qual procurou-se estudar a estabilidade e o desenvolvimento de técnicas para obter estimativas uniformes de atratores, foram utilizados aqui.

Como aplicação, foram feitos alguns exemplos e simulações

No capítulo 03 estudamos inicialmente a estabilidade de sistemas lineares de equações de diferenças $X^{\prime}=A X$, onde $A$ é uma matriz (real ou complexa), e a partir disso procuramos obter informação sobre o sistema não linear $X^{\prime}=A X+f(n)$.

Uma matriz de ordem $m$ é dita estável se o seu raio espectral $r(A)$ é estritamente menor do que 1. Procuramos estudar algoritmos e critérios computacionais para decidir se uma matriz é estável ou não, como por exemplo, o critério de Schur-Cohn. Estudaremos um teorema de Liapunov que dá condições necessárias e suficientes para que uma determinada matriz seja 
estável, assim como o critério de Sylvester para matrizes definidas positivas.

Em seguida vimos as equações de diferenças lineares de m-ésima ordem e sua equivalência com os sistemas de equações de diferenças finitas lineares através de suas matrizes companheiras.

A fórmula da variação das constantes será uma ferramenta de muita utilidade para o estudo do comportamento assintótico de soluções de equações não lineares da forma $x^{\prime}=A x+f(x)$.

$\mathrm{Na}$ teoria de sistemas dinâmicos, o conceito de controlabilidade surgiu naturalmente durante o desenvolvimento inicial de controle ótimo, no final dos anos 1950, e foi descoberto independentemente por matemáticos e engenheiros nos Estados Unidos e na URSS.

No capítulo 4 apresentamos algumas simulações utilizando Matlab, as quais mostram como a sincronização pode ser utilizada em sistemas de comunicação. O sistema de Lorenz foi utilizado. Esta parte está relacionada com o trabalho que foi desenvolvido por GAMEIRO RODRIGUES (veja [30]) para sistemas com variável contínua. 



\section{CAPÍTULO}

\section{Preliminares}

Neste trabalho, assumiremos sempre que $T: \mathbb{R}^{m} \rightarrow \mathbb{R}^{m}$ é uma aplicação contínua.

Dada uma sequiência $x: \mathbb{N} \rightarrow \mathbb{R}^{m}$, definimos $x^{\prime}$ pela regra $x^{\prime}(n)=x(n+1)$; e a primeira diferença de $x$ é dada por $\dot{x}(n):=x(n+1)-x(n)$ ou $\dot{x}=x^{\prime}-x$.

Correspondendo ao teorema fundamental do cálculo, temos $\sum_{k=j}^{n} \dot{x}(k)=x(n+1)-x(j)$.

Se $y(n+1)=\sum_{k=j}^{n} x(k)$, então $\dot{y}(n)=x(n)$.

\subsection{Equações de diferenças}

A equação de diferenças homogênea de primeira ordem é dada por

$$
x(n+1)=T(x(n)),
$$

para todo $n \in \mathbb{N}$, ou abreviadamente, $x^{\prime}=T(x)$. Uma solução dessa equação é uma função (seqüência) $x: \mathbb{N} \rightarrow \mathbb{R}^{m}$ que a satisfaz para todo $n$ natural.

Teorema 1.1.1 (Teorema de existência e unicidade de soluções).

O problema de valor inicial

$$
\left\{\begin{array}{l}
x^{\prime}=T(x) \\
x(0)=x_{0}
\end{array}\right.
$$

admite uma única solução para cada $x_{0} \in \mathbb{R}^{m}$. De fato, tomando $T^{0}=I d$ e $T^{n+1}=$ $T \circ T^{n}$, obtemos um algoritmo definindo a função $x$, que pode ser expressa pela seqüência $\left(x_{0}, T\left(x_{0}\right), T^{2}\left(x_{0}\right), T^{3}\left(x_{0}\right), T^{4}\left(x_{0}\right), \ldots\right)$. 


\subsection{Sistemas dinâmicos discretos}

Definição 1.2.1. Um sistema semidinâmico discreto em $\mathbb{R}^{m}$ é uma função $\Pi: \mathbb{N} \times \mathbb{R}^{m} \rightarrow \mathbb{R}^{m}$ satisfazendo:

\section{1. П é contínua.}

2. $\Pi(0, x)=x$, para todo $x \in \mathbb{R}^{m}$.

3. (Propriedade de semigrupo)

$$
\Pi(m, \Pi(n, x))=\Pi(m+n, x) \text {, para todo } m, n \in \mathbb{N} \text { e para todo } x \in \mathbb{R}^{m} .
$$

Se trocarmos $\mathbb{N}$ por $\mathbb{Z}$ (no caso em que $T$ é inversível) na definição 1.2.1, dizemos que $\Pi$ é um sistema dinâmico discreto.

Toda equação de diferenças define o sistema semidinâmico $\Pi(n, x):=T^{n}(x) ; x \in \mathbb{R}^{m}, n \in \mathbb{N}$. Se $T$ for inversível, então podemos associar essa equação de diferenças ao sistema dinâmico $\Pi(n, x):=T^{n}(x)$, onde $T^{n}(x):=\left(T^{-n}\right)^{-1}$ se $n<0$.

Reciprocamente, todo sistema semidinâmico está associado a equação de diferenças $x^{\prime}=$ $T(x):=\Pi(1, x)$, e por indução finita, se $T^{r}(x)=\Pi(r, x)$ para algum $r \geq 1$ temos que $T^{r+1}(x)=$ $T\left(T^{r}(x)\right)=T(\Pi(r, x))=\Pi(1, \Pi(r, x))=\Pi(r+1, x)$, portanto $T^{n}(x)=\Pi(n, x)$ para todo $n \in \mathbb{N}$.

\subsection{Invariância}

Definição 1.3.1. Seja $H$ um subconjunto de $\mathbb{R}^{m}$ e seja $T: \mathbb{R}^{m} \rightarrow \mathbb{R}^{m}$.

1. H é dito positivamente invariante (com respeito ao operador $T$ ) se $T(H) \subseteq H$, isto é, se $x \in H$ então $T(x) \in H$.

2. $H$ é dito negativamente invariante se $T(H) \supseteq H$, isto é, para todo $y \in H$, existe $x \in H$ tal que $T(x)=y$.

3. Um subconjunto $H$ de $\mathbb{R}^{m}$ que é positivamente invariante e negativamente invariante é dito invariante. Noutras palavras, $H$ é invariante se $T(H)=H$.

\section{Propriedades:}

A reunião de uma família qualquer de conjuntos positivamente invariantes é um conjunto positivamente invariante. Idem para conjuntos invariantes e conjuntos negativamente invariantes. 
A intersecção de uma família qualquer de conjuntos positivamente invariantes é um conjunto positivamente invariante. Em geral a intersecção de dois conjuntos invariantes (ou negativamente invariantes) nem sempre resulta num conjunto invariante (negativamente invariante); no entanto, se $T$ for injetora, então a intersecção de dois conjuntos negativamente invariantes é um conjunto negativamente invariante.

$\varnothing \mathrm{e} \mathbb{R}^{m}$ são positivamente invariantes.

Proposição 1.3.1. Se $H \subseteq \mathbb{R}^{m}$ é $T$-invariante, onde $T: \mathbb{R}^{m} \rightarrow \mathbb{R}^{m}$ é contínua, então:

a) Se $M \subset H$ é positivamente invariante, então $H-M$ é negativamente invariante. Se $T_{\mid H}$ for inversível, então vale a recíproca.

b) $S e T_{\mid H}$ é inversível e $M \subset H$ é invariante, então $H-M$ também é invariante.

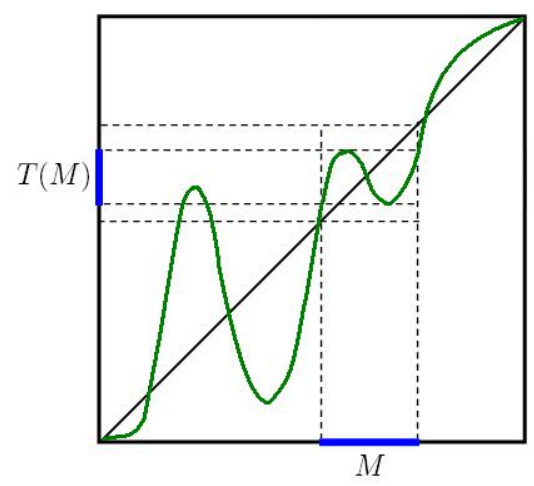

Figura 1.1:

\section{Prova:}

Como $M$ é positivamente invariante temos que $T(M) \subset M$. Assim, $T(H-M) \supset T(H)-$ $T(M)=H-T(M) \supset H-M, \log o H-M$ é negativamente invariante.

Se $T$ for inversível e $H-M$ é negativamente invariante, $H-M \subset T(H-M)=H-T(M)$, $\operatorname{logo} M \supset T(M)$.

b)

Se $M$ é invariante, Por a) temos que $H-M$ é negativamente invariante. Portanto, resta apenas mostrar que $H-M$ é positivamente invariante.

Seja $x \in H-M$ e suponha que $T(x) \in M$, como $M$ é invariante, existe $y \in M$ tal que $T(y)=$ $T(x)$, mas como $T_{\mid H}$ é inversível, temos que $y=x$, o que é uma contradição, logo $T(x) \in H-M$ e portanto $H-M$ é positivamente invariante. 
Observação 1.3.1 (Prolongamento ou extensão de soluções).

Se $\left(x_{0}, T\left(x_{0}\right), T^{2}\left(x_{0}\right), T^{3}\left(x_{0}\right), \ldots\right)$ é solução de (1.1) e $x_{-1} \in T^{-1}\left(x_{0}\right)$ (imagem inversa de $x_{0}$ ), então a solução pode ser prolongada a $\left(x_{-1}, x_{0}, T\left(x_{0}\right), T^{2}\left(x_{0}\right), T^{3}\left(x_{0}\right), \ldots\right)$.

Um prolongamento é dito maximal se estiver definido para todo $\mathbb{Z}$, ou então se $\left(x_{-n}, \cdots, x_{-2}, x_{-1}, x_{0}, T\left(x_{0}\right), T^{2}\left(x_{0}\right), T^{3}\left(x_{0}\right), \ldots\right)$ for um prolongamento e $T^{-1}\left(x_{-n}\right)=\varnothing$.

Se T for injetiva então o prolongamento em H é sempre único, e se T for sobrejetiva, então toda solução admite um prolongamento para todo $z \in \mathbb{Z}$.

Definição 1.3.2. O conjunto $\gamma^{+}\left(x_{0}\right):=\left\{T^{n}\left(x_{0}\right): x_{0} \in \mathbb{R}^{m}, n \in \mathbb{N}\right\}$, isto é, a imagem da solução de (1.1) chama-se órbita positiva (ou trajetória positiva) de $x_{0}$ e é positivamente invariante. Se $T$ for inversível, definimos a órbita negativa de $x_{0}$ como sendo o conjunto $\gamma^{-}\left(x_{0}\right):=\left\{T^{-n}\left(x_{0}\right)\right.$ : $n \in \mathbb{N}\}$ que é negativamente invariante, e neste caso, $\gamma^{+}\left(x_{0}\right) \cup \gamma^{-}\left(x_{0}\right)$ é a órbita completa (ou simplesmente órbita) de $x_{0}$ e é invariante.

Proposição 1.3.2. Um conjunto $H \subset \mathbb{R}^{m}$ é negativamente invariante se e somente se toda órbita iniciando em $H$ tem um prolongamento em $H$ para todo $n \in \mathbb{Z}$.

\section{Prova:}

Seja $x_{0} \in H . \quad$ Como $T(H) \supset H$ temos que existe $x_{-1} \in H$ tal que $T\left(x_{-1}\right)=x_{0}$; e por indução, dado $x_{-n} \in H$, existe $x_{-n-1} \in H$ tal que $T\left(x_{-n-1}\right)=x_{-n}$. Portanto $\left(\cdots, x_{-n}, \cdots, x_{-1}, x_{0}, T\left(x_{0}\right), T^{2}\left(x_{0}\right), \cdots\right)$ é um prolongamento de $\left(T^{n}\left(x_{0}\right)\right)$ para todo $n \in \mathbb{Z}$.

Reciprocamente, se toda órbita que inicia em $H$ tem um prolongamento em $H$, então para todo $y \in H$, existe $x \in H$ tal que $T(x)=y, \operatorname{logo} H$ é negativamente invariante.

Proposição 1.3.3. O fecho de um conjunto positivamente invariante é positivamente invariante.

\section{Prova:}

Se $H \subset \mathbb{R}^{m}$ é positivamente invariante, $T(H) \subset H$, portanto $\overline{T(H)} \subset \bar{H}$. Como $T$ é contínua, $T(\bar{H}) \subset \overline{T(H)}, \log \mathrm{T}(\bar{H}) \subset \bar{H}$.

Não é verdade em geral que o fecho de um conjunto negativamente invariante é negativamente invariante, por exemplo, se $f(x)=\left[e^{-x}+1\right] \operatorname{sen}^{2}\left(\frac{\pi x}{2}\right)+\left[\frac{x}{2}+2\right] \cos ^{2}\left(\frac{\pi x}{2}\right)$, temos que $(1,+\infty)$ é $f$-negativamente invariante mas $[1,+\infty)=\overline{(1,+\infty)}$ não é negativamente invariante, visto que $f([1,+\infty))=(1,+\infty)$.

No entanto, temos o seguinte resultado:

Proposição 1.3.4. O fecho de um conjunto negativamente invariante limitado é negativamente invariante. 


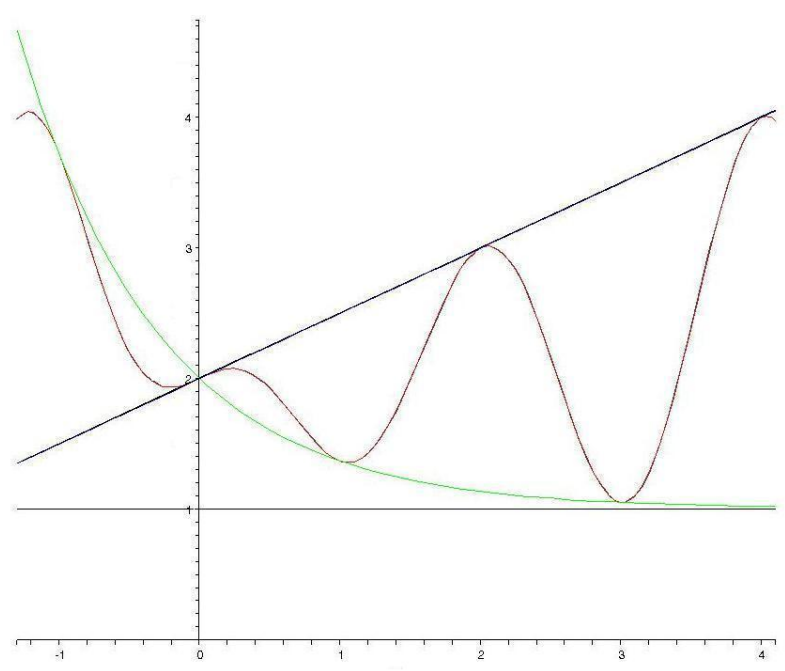

Figura 1.2:

\section{Prova:}

Dado $x \in \bar{H}$, existe $\left(x_{n}\right) \operatorname{com} x_{n} \rightarrow x$ e $x_{n} \in H$ para todo $n \in \mathbb{N}$. Como $H$ é $T$-negativamente invariante, temos que $x_{n} \in T(H)$, assim, existe uma sequiência $\left(y_{n}\right)$ tal que $x_{n}=T\left(y_{n}\right)$.

Como $H$ é limitado, $\bar{H}$ é compacto e assim existe uma subsequiência $y_{n_{k}}$ convergente, digamos $y_{n_{k}} \rightarrow y \in \bar{H}$. Pela continuidade de $T, x=\lim _{n_{k} \rightarrow \infty} x_{n_{k}}=\lim _{n_{k} \rightarrow \infty} T\left(y_{n_{k}}\right)=T\left(\lim _{n_{k} \rightarrow \infty} y_{n_{k}}\right)=$ $T(y) \Rightarrow x \in T(\bar{H})$.

Definição 1.3.3. Um conjunto $H \in \mathbb{R}^{m}$ que é fechado e invariante (com respeito a algum operador $\left.T: \mathbb{R}^{m} \rightarrow \mathbb{R}^{m}\right)$, é dito invariantemente conexo se não existem dois conjuntos fechados não vazios, invariantes e disjuntos $F_{1}$ e $F_{2}$ tais que $H=F_{1} \sqcup F_{2}$.

No que segue, é interessante observarmos que se um conjunto não vazio $H \in \mathbb{R}^{m}$ é (fechado e) invariante, faz sentido definirmos o sistema semidinâmico $\Pi: \mathbb{N} \times H \rightarrow H, \Pi(n, x)=T_{\mid H}^{n}(x)$.

Se existirem dois fechados invariantes, disjuntos e não vazios $F_{1}$ e $F_{2}$ tais que $H=F_{1} \sqcup F_{2}$, pela invariância de $F_{1}$, se uma solução de (1.1) inicia em $F_{1}$ então ela está totalmente contida em $F_{1}$. Analogamente, se uma solução de (1.1) inicia em $F_{2}$ então ela está totalmente contida em $F_{2}$. Assim, num certo sentido temos dois sistemas semidinâmicos: $\Pi_{1}: \mathbb{N} \times F_{1} \rightarrow F_{1}, \Pi_{1}(n, x)=$ $T_{\mid F_{1}}^{n}(x)$ e $\Pi_{2}: \mathbb{N} \times F_{2} \rightarrow F_{2}, \Pi_{2}(n, x)=T_{\mid F_{2}}^{n}(x)$, e a dinâmica de $\Pi$ em $F_{1}$ pode inclusive ser diferente da dinâmica de $\Pi$ em $F_{2}$.

Exemplo 1.3.1. Se $H \subset \mathbb{R}^{m}$ é fechado, invariante e conexo, então $H$ é invariantemente conexo.

De fato, Se H não fosse invariantemente conexo, existiriam dois fechados disjuntos e não vazios $F_{1}$ e $F_{2}$ tais que $H=F_{1} \sqcup F_{2}$. Como $\mathbb{R}^{m}$ é um espaço topológico normal, existem $B_{1} e$ 

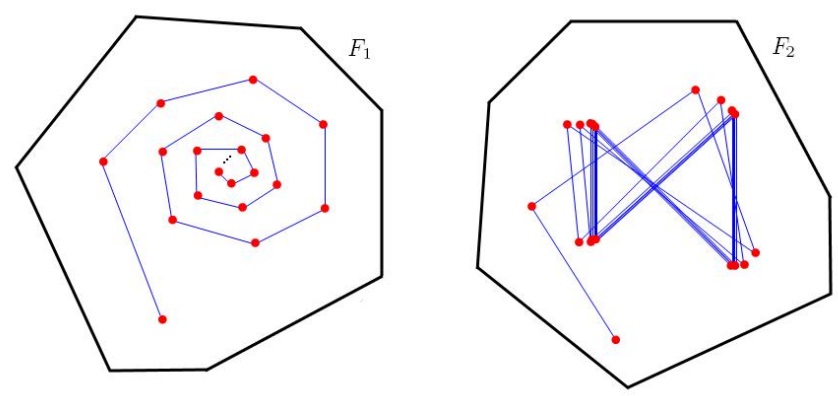

Figura 1.3:

$B_{2}$ subconjuntos abertos e disjuntos de $\mathbb{R}^{m}$ tais que $F_{1} \subset B_{1}$ e $F_{2} \subset B_{2}$. Mas então $H \subset B_{1} \sqcup B_{2}$ com $B_{1} \neq \varnothing$ e $B_{2} \neq \varnothing$. Absurdo, pois $H$ é conexo.

A recíproca desse resultado é obviamente falsa. De fato, seja $f: \mathbb{R} \rightarrow \mathbb{R}$ dada pela regra $f(x)=-x+1$ e seja $H=\left[0, \frac{1}{3}\right] \cup\left[\frac{2}{3}, 1\right]$. H é invariantemente conexo, apesar de não ser conexo.

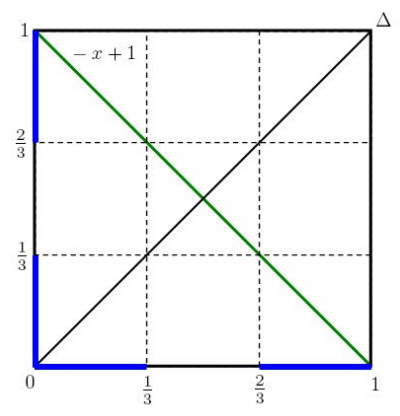

Figura 1.4:

Definição 1.3.4. Uma solução de (1.1) é dita periódica (ou cíclica) se existe $k \in \mathbb{N}$ tal que $T^{k}\left(x_{0}\right)=x_{0}$, e o menor $k$ com essa propriedade é dito período da solução periódica. Analogamente definimos órbita periódica, cujos elementos são ditos pontos periódicos. Indicamos o conjunto dos pontos $T$-periódicos por $\operatorname{Per}(T)$ e o conjunto dos pontos periódicos de período $k$ por $\operatorname{Per}(T, k)$. Se $k=1$, isto é, $T\left(x_{0}\right)=x_{0}$ então $x_{0}$ é dito ser um ponto fixo (ou ponto invariante, ou ainda ponto de equilíbrio) de $T$.

Proposição 1.3.5. Se H é um conjunto invariante com um número finito de elementos, então $T_{\mid H}$ é inversível. Em particular, toda solução periódica de (1.1) admite um prolongamento para todo $n \in \mathbb{Z}$.

\section{Prova:}


Pela invariância de $H$, temos que $T(H)=H$, logo $T_{\mid H}$ é sobrejetora. Como $H$ tem um número finito de elementos, temos que $T_{\mid H}$ também é injetora.

Se $T^{n}\left(x_{0}\right), \quad x_{0} \in \mathbb{R}^{m}$ é uma solução periódica de período $k$ de (1.1), temos que $H=\left\{x_{0}, T\left(x_{0}\right), T^{2}\left(x_{0}\right), \cdots, T^{k-1}\left(x_{0}\right)\right\}$ é um conjunto invariante com um número finito de elementos. Segue que $T_{H}$ é inversível, e a existência (e unicidade) do prolongamento da solução periódica (em $H$ ) para todo $\mathbb{Z}$ segue da observação 1.3.1, ou então da Proposição 1.3.2. Explicitamente, um prolongamento é $\left\{\cdots, x_{0}, T\left(x_{0}\right), T^{2}\left(x_{0}\right), \cdots, T^{k-1}\left(x_{0}\right), x_{0}, T\left(x_{0}\right), T^{2}\left(x_{0}\right), \cdots, T^{k-1}\left(x_{0}\right), \cdots\right\}$.

Proposição 1.3.6. Um conjunto invariante com um número finito de elementos é invariantemente conexo se e somente se for uma órbita periódica. Em particular, todo conjunto invariante com um número finito de elementos é reunião disjunta de órbitas periódicas.

\section{Prova:}

Suponha que H não seja uma órbita periódica. Se \#H=m, então dado $x \in H$ existe $n<m$ tal que $M=\left\{x, T(x), T^{2}(x) \cdots T^{n-1}(x)\right\}$ é uma órbita periódica, e portanto um conjunto invariante. Pela Proposição 1.3.5 $T_{\mid H}$ é inversível, logo pela Proposição 1.3.1 $H-M$ também é invariante. Mas então $H$ e $H-M$ são dois fechados invariantes disjuntos e não vazios tais que $H=M \sqcup$ $H-M$. Absurdo pois H é invariantemente conexo.

Se $H$ é uma órbita periódica de período $k$, digamos $H=\left\{x, T(x), T^{2}(x), \cdots T^{k-1}(x)\right\}$ e se $M$ é um subconjunto invariante não vazio de $H$, então existe $n_{0}, 0 \leq n_{0}<k$ tal que $T^{n_{0}}(x) \in M$. Como $M$ é invariante, $T^{n_{0}+n}(x) \in M$ para todo $n \in \mathbb{N}$. Visto que $T^{k}(x)=x$, temos que $H=$ $\left\{T^{n_{0}}(x), T^{n_{0}+1}(x), \cdots, T^{n_{0}+k-1}(x)\right\} \subset M . \log \mathrm{O} M=H$ e $H$ é invariantemente conexo.

Se $H \subset \mathbb{R}^{m}$ é um conjunto invariante com um número finito de elementos, tomando $x_{1} \in H$ arbitrariamente, temos que existe $n_{1} \leq m:=\# H$ tal que o conjunto $\gamma\left(x_{1}\right)=$ $\left\{x_{1}, T\left(x_{1}\right), T^{2}\left(x_{1}\right), \cdots, T^{n_{1}-1}\left(x_{1}\right)\right\}$ é uma órbita periódica.

Se $n_{1}=m$ a afirmação segue, senão, existe $x_{2} \in H-\gamma\left(x_{1}\right)$ que é um conjunto finito, portanto existe $n_{2} \leq m$ tal que o conjunto $\gamma\left(x_{2}\right)=\left\{x_{2}, T\left(x_{2}\right), T^{2}\left(x_{2}\right), \cdots, T^{n_{2}-1}\left(x_{2}\right)\right\}$ é uma órbita periódica.

Se $n_{1}+n_{2}=m$ a afirmação segue, senão, existe $x_{3} \in H-\left(\gamma\left(x_{1}\right) \sqcup \gamma\left(x_{2}\right)\right)$ que é um conjunto finito, portanto existe $n_{3} \leq m$ tal que o conjunto $\gamma\left(x_{3}\right)=\left\{x_{3}, T\left(x_{3}\right), T^{2}\left(x_{3}\right), \cdots, T^{n_{3}-1}\left(x_{3}\right)\right\}$ é uma órbita periódica.

Prosseguindo assim, como $H$ é finito, esse processo tem que acabar. Assim, obteremos que $H=\gamma\left(x_{1}\right) \sqcup \gamma\left(x_{2}\right) \sqcup \cdots \sqcup \gamma\left(x_{k}\right)$, onde os $\gamma\left(x_{i}\right)$ são órbitas periódicas duas a duas disjuntas.

O estudo dos pontos fixos tem grande importância para se determinar os pontos de equilíbrio de sistemas dinâmicos discretos, além de constituir por si só um objeto de pesquisa intensa. A 
procura de uma solução de uma equação do tipo $f(x)=b$ reduz-se a procura de um ponto fixo para a aplicação $\xi(x)=f(x)+x-b$. De fato, $\xi(x)=x \Leftrightarrow f(x)=b$.

Sob certas hipóteses sobre um operador $T$ e um subconjunto $H$ que é $T$-positivamnete invariante, alguns resultados garantem a existência de um ponto fixo para $T$ em $H$.

Teorema 1.3.1 (Ponto fixo de Banach).

Seja $F$ um subconjunto fechado e f-positivamente invariante de um espaço métrico completo, onde $f: F \rightarrow F$ uma contração, isto é, existe $\lambda \in \mathbb{R}$ com $0 \leq \lambda<1$ tal que $d(f(x), f(y)) \leq \lambda d(x, y)$. Então $f$ tem um único ponto fixo, o qual é atrator.

Teorema 1.3.2 (Ponto fixo de Brower).

Seja $T: \mathbb{R}^{m} \rightarrow \mathbb{R}^{m}$ uma aplicação contínua, $H \subset \mathbb{R}^{m}$ homeomorfo a $B[0,1]=\overline{B(0,1)} e$ T-positivamente invariante. Então existe $x \in H$ tal que $T(x)=x$.

Exemplo 1.3.2. Se $f: \mathbb{R} \rightarrow \mathbb{R}, f(x)=-x+1$ e $H=\left[0, \frac{1}{3}\right] \cup\left[\frac{2}{3}, 1\right]$ como no exemplo 1.3.1, então $H$ é um conjunto fechado e invariante, porém sem ponto fixo.

\subsection{Conjuntos limite}

Se $\left(x, T(x), T^{2}(x), \ldots\right)$ é convergente, então $a=\lim _{n \rightarrow \infty} T^{n}(x)$ é um ponto fixo de $T$. Mais geralmente temos:

Definição 1.4.1 (Birkhoff).

Dado $x \in \mathbb{R}^{m}$, seja $\left(T^{n}(x)\right)$ a solução de (1.1) que inicia em $x$. Define-se o conjunto $\omega(x):=$ $\left\{q \in \mathbb{R}^{m}: \exists n_{k} \rightarrow \infty e T^{n_{k}}\left(x_{0}\right) \rightarrow q, k \rightarrow \infty\right\}$. $\omega(x)$ é dito conjunto $\omega$-limite do ponto $x$.

Se $y \in \gamma^{+}(x)$, então $\omega(x)=\omega(y)$, isto é, o conjunto $\omega$-limite é uma propriedade da órbita de um ponto, e não desse ponto. De fato, se $y \in \gamma^{+}(x)$, temos que $y=T^{k}(x)$ e se $z \in \omega(x)$, temos que existe uma subseqüência $T^{n_{i}}(x) \rightarrow z, n_{i}>k, \operatorname{assim} z=\lim _{n_{i} \rightarrow \infty} T^{n_{i}}(x)=$ $\lim _{n_{i} \rightarrow \infty} T^{n_{i}-k+k}(x)=\lim _{n_{i} \rightarrow \infty} T^{n_{i}-k}\left(T^{k}(x)\right)=\lim _{n_{i} \rightarrow \infty} T^{n_{i}-k}(y)$, portanto $\omega(x) \subset \omega(y)$ e a outra inclusão se verifica analogamente. Assim, podemos definir o conjunto $\omega$-limite de uma órbita.

Observação 1.4.1. Equivalentemente, temos que $\omega(x):=\bigcap_{j=0}^{\infty} \overline{\bigcup_{n=j}^{\infty} T^{n}(x)}$. Em particular, $\omega(x) e ́$ fechado.

\section{Prova:}

Seja $y \in \omega\left(x_{0}\right)$. Dado $j>0$ existe uma subseqüência $T^{n_{k}}(x)$ de $T^{n}(x), n_{k}>j$, que converge para $y$. Logo, $y \in \overline{\bigcup_{n=j}^{\infty} T^{n}(x)}$. Como $j$ é arbitrário, $y \in \bigcap_{j=0}^{\infty} \overline{\bigcup_{n=j}^{\infty} T^{n}(x)}$. 
Provemos agora a outra inclusão. Sejam $A_{j}=\bigcup_{n=j}^{\infty} T^{n}(x)$ e $y \in \bigcap_{j=0}^{\infty} \overline{A_{j}}$. Então $y \in \overline{A_{0}}$, $\operatorname{logo}$ existe uma sequiência em $A_{0}$ que converge para $y$. Seja $y_{0}=T^{n_{0}}(x)$ um termo dessa sequiência tal que $\left|y-y_{0}\right|<1$, para algum $n_{0} \geq 0$.

Como $y \in \overline{A\left(n_{0}+1\right)}$, existe uma seqüência em $A_{n_{0}+1}$ convergindo para $y$. Seja $y_{1}=T^{n_{1}}(x)$ um termo dessa sequiência tal que $\left|y-y_{1}\right|<\frac{1}{2}$ para algum $n_{1}>n_{0}$.

Como $y \in \overline{A\left(n_{1}+1\right)}$, existe uma seqüência em $A_{n_{1}+1}$ convergindo para $y$. Seja $y_{1}=T^{n_{1}}(x)$ um termo dessa seqüência tal que $\left|y-y_{1}\right|<\frac{1}{2^{2}}$ para algum $n_{2}>n_{1}>n_{0}$.

Prosseguindo assim, criamos uma seqüência $y_{k} \in \mathbb{R}^{m}$ tal que $\left|y-y_{k}\right|<\frac{1}{2^{k}}, T^{n_{k}}(x)$ e $n_{k}>$ $n_{k-1}$, portanto $y_{k}=T^{n_{k}}(x) \rightarrow x$ quando $k \rightarrow \infty$, e assim, $y \in \omega\left(x_{0}\right)$.

Existem algumas razões para estarmos interessados em estudar o que acontece com $T^{n}(x)$ para valores grandes de $n$. isso concerne com o comportamento assintótico de $T^{n}(x)$, assunto tratado pela teoria da estabilidade. No próximo capítulo veremos como obter informação sobre conjuntos limites de órbitas utilizando funções de Liapunov.

Teorema 1.4.1. Se existe $W \subset \mathbb{R}^{m}$ compacto tal que $T^{n}(x) \in W$ para todo $n \in \mathbb{N}$, então $\omega(x)$ tem as seguintes propriedades:

1. $\omega(x)$ é não vazio;

2. $\omega(x)$ é compacto;

3. $\omega(x)$ é invariante;

4. $\omega(x)$ é invariantemente conexo;

5. $\omega(x)$ é o menor conjunto fechado do qual $T^{n}(x)$ se aproxima quando $n \rightarrow \infty$.

\section{Prova:}

1. $\omega(x)$ é não vazio:

Como $\left\{T^{n}(x)\right\} \subset W$ que é compacto, segue que $\left\{T^{n}(x)\right\}$ tem um ponto limite, logo $\omega(x) \neq$ $\varnothing$.

2. $\omega(x)$ é compacto:

Como $\omega(x) \subset W$ que é compacto, é suficiente verificar que $\omega(x)$ é fechado. Isso segue da observação 1.4.1.

3. $\omega(x)$ é invariante: 
Mostremos primeiro que $T(\omega(x)) \subseteq \omega(x)$, isto é, $\omega(x)$ é positivamente invariante. Como $T$ é contínua, temos:

$$
\begin{gathered}
T(\omega(x))=T\left(\bigcap_{j=0}^{\infty} \overline{\bigcup_{n=j}^{\infty} T^{n}(x)}\right) \subset \bigcap_{j=0}^{\infty} T\left(\overline{\left(\bigcup_{n=j}^{\infty} T^{n}(x)\right.}\right) \subset \bigcap_{j=0}^{\infty} \overline{T\left(\bigcup_{n=j}^{\infty} T^{n}(x)\right)}= \\
\bigcap_{j=0}^{\infty} \overline{\left(\bigcup_{n=j}^{\infty} T^{n+1}(x)\right)}=\bigcap_{j=0}^{\infty} \overline{\left(\bigcup_{n=j+1}^{\infty} T^{n}(x)\right)} \subset \bigcap_{j=0}^{\infty} \overline{\left(\bigcup_{n=j}^{\infty} T^{n}(x)\right)}=\omega(x) .
\end{gathered}
$$

Observe que aqui não se usou o fato de $\left(T^{n}(x)\right)$ ser limitada.

Mostremos agora que $T(\omega(x)) \supseteq \omega(x)$, isto é, $\omega(x)$ é negativamente invariante.

Dado $y \in \omega(x)$, seja $T^{n_{i}}(x)$ tal que $T^{n_{i}}(x) \rightarrow y$.

Como $T^{n}(x)$ é limitada temos que $T^{n_{i}-1}(x)$ é limitada e portanto existe $T^{n_{i_{k}}-1}(x)$ convergente; digamos $T^{n_{i_{k}}-1}(x) \rightarrow z \in \omega(x)$. Pela continuidade de $T$ :

$$
y=\lim _{k \rightarrow \infty} T^{n_{i_{k}}}(x)=\lim _{k \rightarrow \infty} T\left(T^{n_{i_{k}}-1}(x)\right)=T\left(\lim _{k \rightarrow \infty} T^{n_{i_{k}}-1}(x)\right)=T(z) .
$$

Logo $T(\omega(x)) \supseteq \omega(x)$.

4. $\omega(x)$ é invariantemente conexo:

Suponha por contradição que $\omega(x)$ é igual a reunião de dois conjuntos fechados invariantes não vazios e disjuntos $\omega_{1}$ e $\omega_{2}$.

Como $\omega(x)$ é compacto, segue que $\omega_{1}$ e $\omega_{2}$ também são compactos. Como $\mathbb{R}^{m}$ é um espaço topológico normal, existem dois abertos disjuntos $U_{1}$ e $U_{2}$ tais que $\omega_{1} \subset U_{1}$ e $\omega_{2} \subset U_{2}$.

Como $T$ é contínua, existe um conjunto aberto $B_{1}$ tal que $\omega_{1} \subset B_{1}$ e $T\left(B_{1}\right) \subset U_{1}$. De fato, tomando $B_{1}:=T^{-1}\left(U_{1}\right)$, temos que $B_{1}$ é aberto, $T\left(B_{1}\right)=T\left(T^{-1}\left(U_{1}\right)\right) \subset U_{1}$ e, como $\omega_{1}$ é invariante, $\omega_{1} \subset T^{-1}\left(T\left(\omega_{1}\right)\right)=T^{-1}\left(\omega_{1}\right) \subset T^{-1}\left(U_{1}\right)=B_{1}$, pois $\omega_{1} \subset B_{1}$.

Como por hipótese $\omega(x)=\omega_{1} \sqcup \omega_{2}$ e visto que $\omega_{1} \subset U_{1} \cap B_{1}$ e $\omega_{2} \subset U_{2}$, então existe $n_{0} \in \mathbb{N}$ tal que $T^{n}(x) \in\left(U_{1} \cap B_{1}\right) \cup U_{2}$ para todo $n>n_{0}$.

Como $\omega_{1} \neq \varnothing$ podemos supor que existe $n_{0}$ tal que $T^{n_{0}}(x) \in U_{1} \cap B_{1}$. Suponha que exista $n_{1}>n_{0}$ tal que $T^{n_{1}}(x) \in U_{1} \cap B_{1}$ e $T^{n_{1}+1}(x) \in U_{2}$. Mas como $T\left(B_{1}\right) \subset U_{1}$, temos $T^{n_{1}+1}(x)=$ $T\left(T^{n_{1}}(x)\right) \in T\left(U_{1} \cap B_{1}\right) \subset U_{1}$, ou seja $T^{n_{1}+1}(x) \in U_{1}$, o que é uma contradição, pois $U_{1} \cap U_{2}=$ $\varnothing, \operatorname{logo} \omega(x)$ é invariantemente conexo.

5. $\omega(x)$ é o menor conjunto fechado do qual $T^{n}(x)$ se aproxima quando $n \rightarrow \infty$ : 
Seja $F$ um subconjunto fechado de $\mathbb{R}^{m}$ do qual $T^{n}(x)$ se aproxima quando $n \rightarrow \infty$ e seja $y \in \omega\left(x_{0}\right)$. Então existe uma subseqüência $T^{n_{i}}(x) \rightarrow y$. Se $\{x\}, S \subset \mathbb{R}^{m}$ definimos $\rho(x, S):=$ $\inf _{y \in S}|x-y|$. Assim, $\rho\left(T^{n_{i}}(x), F\right) \rightarrow \rho(y, F)$. Como $\rho\left(T^{n_{i}}(x), F\right) \rightarrow 0$ pois $T^{n}\left(x_{0}\right) \rightarrow F$ quando $n \rightarrow \infty$, temos que $\rho(y, F)=0$, e portanto $y \in F$ pois $F$ é fechado e assim, $\omega(x) \subset F$.

Observe que se nada for dito sobre a inclusão de $\omega(x)$ em um subconjunto compacto de $\mathbb{R}^{m}$, ou o que é equivalente, sobre sua limitação, podemos afirmar apenas que $\omega(x)$ é fechado e positivamente invariante.

Observação 1.4.2. Se $T^{n}(x)$ é limitado, então $\omega(x)$ é invariantemente conexo. No entanto, não é possível afirmar que $\omega(x)$ seja conexo. Por exemplo se $R_{\alpha}: \mathbb{R}^{2} \rightarrow \mathbb{R}^{2}$ é a rotação de um ângulo $\alpha$ (com relação a origem) e $x \in \mathbb{S}^{1}=\left\{x \in \mathbb{R}^{2}:|x|=1\right\}$; se $\alpha$ for múltiplo racional de $\pi=3.14159 \ldots$ isto é, $\alpha=\frac{p}{q} \pi$, onde $p \in \mathbb{Z}, q \in \mathbb{Z}-\{0\}$ (e podemos supor também que $p>0 e$ $m d c\{p, q\}=1)$, então $R_{\alpha}^{q}(x)=x$, logo $\omega(x)$ é uma órbita periódica e portanto desconexo; mas se $\alpha$ não for múltiplo racional de $\pi$ então $\omega(x)=\mathbb{S}^{1}$ que é conexa (veja Katok, [17]).

Definição 1.4.2. Se $H \subset \mathbb{R}^{m}$, definimos $\Omega(H):=\bigcap_{j=0}^{\infty} \overline{\bigcup_{n=j}^{\infty} T^{n}(H)}$, em particular, $\Omega(H)$ é fechado.

Proposição 1.4.1. Se $H \subset \mathbb{R}^{m}$ então $y \in \Omega(H)$ se, e somente se, existirem seqüências $n_{j}$ em $\mathbb{N}$ $e\left(y_{j}\right)$ em $H$ tais que $T^{n_{j}}\left(y_{j}\right) \rightarrow$ y quando $j \rightarrow \infty$.

\section{Prova:}

$\Rightarrow$ Suponha que existam seqüências $n_{j}$ em $\mathbb{N}$ e $\left(y_{j}\right)$ em $H$ tais que $T^{n_{j}}\left(y_{j}\right) \rightarrow y$ quando $j \rightarrow \infty$. Suponha sem perda de generalidade que $n_{j}$ é crescente, então dado $i \in \mathbb{N}$, existe $j_{0} \in \mathbb{N}$ tal que $n_{j_{0}}>i$ para qualquer $j>j_{0}$. Assim, $T^{n_{j}}\left(y_{j}\right) \rightarrow y$ e $T^{n_{j}}\left(y_{j}\right) \in T^{n_{j}}(H) \subset \bigcup_{n=i}^{\infty} T^{n}(H)$, dessa forma, $y \in \overline{\bigcup_{n=i}^{\infty} T^{n}(H)}$ para todo $i$ e assim, $y \in \bigcap_{i=0}^{\infty} \overline{\bigcup_{n=i}^{\infty} T^{n}(H)}$. Provemos agora a outra implicação. $\Leftarrow$ Sejam $y \in \Omega(H), A_{j}=\bigcup_{n=j}^{\infty} T^{n}(H)$ e $y \in \bigcap_{j=0}^{\infty} \overline{A_{j}}$, então $y \in \overline{A_{0}}$, logo existe uma seqüência em $A_{0}$ que converge para $y$. Seja $w_{0}=T^{n_{0}}\left(y_{0}\right)$ um termo dessa sequiência tal que $\left|y-w_{0}\right|<1$, para algum $n_{0} \geq 0$ e para algum $y_{0} \in H$.

Como $y \in \overline{A\left(n_{0}+1\right)}$, existe uma seqüência em $A_{n_{0}+1}$ convergindo para $y$. Seja $w_{1}=T^{n_{1}}\left(y_{1}\right)$ um termo dessa sequiência tal que $\left|y-w_{1}\right|<\frac{1}{2}$ para algum $n_{1}>n_{0}$ e para algum $y_{1} \in H$.

Como $y \in \overline{A\left(n_{1}+1\right)}$, existe uma seqüência em $A_{n_{1}+1}$ convergindo para $y$. Seja $w_{2}=T^{n_{2}}\left(y_{2}\right)$ um termo dessa sequiência tal que $\left|y-w_{2}\right|<\frac{1}{2^{2}}$ para algum $n_{2}>n_{1}>n_{0}$ e para algum $y_{2} \in H$.

Prosseguindo assim, criamos uma seqüência $y_{k} \in H$ tal que $\left|y-y_{k}\right|<\frac{1}{2^{k}}, w_{k}=T^{n_{k}}\left(y_{k}\right) \mathrm{e}$ $n_{k}>n_{k-1}, w_{k}=T^{n_{k}}\left(y_{k}\right)$, portanto $w_{k} \rightarrow y$ quando $k \rightarrow \infty$. 
Teorema 1.4.2. Se $H \neq \varnothing$ e existe $W \subset \mathbb{R}^{m}$ compacto tal que $T^{n}(H) \subset W$ para todo $n \in \mathbb{N}$, então $\Omega(H)$ tem as seguintes propriedades:

1. $\Omega(H)$ é não vazio;

2. $\Omega(H)$ é compacto;

3. $\Omega(H)$ é invariante;

4. $\Omega(H)$ é o menor conjunto fechado do qual $T^{n}(H)$ se aproxima quando $n \rightarrow \infty$.

\section{Prova:}

1. $\Omega(H)$ é não vazio:

Se $x \in H$, temos que $T^{n}(x) \in T^{n}(H) \subset W$ que é compacto. Então $\left\{T^{n}(x)\right\}$ tem algum ponto limite em $W$ e assim, $\Omega(H) \neq \varnothing$.

2. $\Omega(H)$ é compacto:

$\Omega(H)$ é fechado pela definição 1.4.2. Como $\Omega(H) \subset W$ que é compacto seque que $\Omega(H)$ é compacto.

3. $\Omega(H)$ é invariante:

Provemos inicialmente que $T(\Omega(H)) \subseteq \Omega(H)$, isto é, $\Omega(H)$ é positivamente invariante. De fato:

$$
\begin{gathered}
T(\Omega(H))=T\left(\bigcap_{j=0}^{\infty} \overline{\bigcup_{n=j}^{\infty} T^{n}(H)}\right) \subset \bigcap_{j=0}^{\infty} T\left(\overline{\left(\bigcup_{n=j}^{\infty} T^{n}(H)\right.}\right) \subset \bigcap_{j=0}^{\infty} T \overline{\left(\bigcup_{n=j}^{\infty} T^{n}(H)\right)}= \\
\bigcap_{j=0}^{\infty} \overline{\left(\bigcup_{n=j}^{\infty} T^{n+1}(H)\right)}=\bigcap_{j=0}^{\infty} \overline{\left(\bigcup_{n=j+1}^{\infty} T^{n}(H)\right)} \subset \bigcap_{j=0}^{\infty} \overline{\left(\bigcup_{n=j}^{\infty} T^{n}(H)\right)}=\Omega(H) .
\end{gathered}
$$

Observe que aqui não se usou o fato de $\left(T^{n}(H)\right)$ ser limitada.

Provemos agora a inclusão $T(\Omega(H)) \supseteq \Omega(H)$, isto é, $\Omega(H)$ é negativamente invariante.

Dado $y \in \Omega(H)$, existem seqüências $y_{j} \in H$ e $n_{j} \in \mathbb{N}$ tais que $T^{n_{j}}\left(y_{j}\right) \rightarrow y$ e $n_{j} \rightarrow \infty$ quando $j \rightarrow \infty$.

$$
y=\lim _{j \rightarrow \infty} T^{n_{j}}\left(y_{j}\right)=\lim _{j \rightarrow \infty} T\left(T^{n_{j}-1}\left(y_{j}\right)\right)=T\left(\lim _{j \rightarrow \infty} T^{n_{j}-1}\left(y_{j}\right)\right)=T(z) .
$$


4. $\Omega(H)$ é o menor conjunto fechado do qual $T^{n}(H)$ se aproxima quando $n \rightarrow \infty$ :

Seja $F$ um subconjunto fechado de $\mathbb{R}^{m}$ tal que $T^{n}(H) \rightarrow F$ quando $n \rightarrow \infty$. Dado $\varepsilon>0$, se $A_{n}:=\left\{\rho(z, F): z \in T^{n}(H)\right\}$, então existe $n_{0}$ tal que $\sup A_{n}<\varepsilon$ para todo $n>n_{0}$.

Se $y \in \Omega(H)$ existem seqüências $y_{j} \in H$ e $n_{j} \geq n_{0}$ tais que $T^{n_{j}}\left(y_{j}\right) \rightarrow \infty$ quando $j \rightarrow \infty$.

Como $T^{n_{j}}\left(y_{j}\right) \in T^{n_{j}}(H), \rho\left(T^{n_{j}}\left(y_{j}\right), F\right) \in A_{n_{j}}$, e como $n_{j} \geq n_{0}$, temos:

$\rho\left(T^{n_{j}}\left(y_{j}\right), F\right)<\varepsilon \Rightarrow \rho(y, F)=\rho\left(\lim _{n \rightarrow \infty} T^{n_{j}}\left(y_{j}\right), F\right)=\lim _{n \rightarrow \infty} \rho\left(T^{n_{j}}\left(y_{j}\right), F\right) \leq \varepsilon . \operatorname{Logo}, \rho(y, F) \leq$ $\varepsilon$ para todo $\varepsilon>0$, o que implica que $y \in \bar{F}=F$. Portanto $\Omega(H) \subset F$.

Proposição 1.4.2. Se existe um conjunto compacto $H \subset \mathbb{R}^{m}$ e $f_{n}: H \rightarrow \mathbb{R}, f_{n}(y):=$ $\rho\left(T^{n}(y), \overline{\bigcup_{x \in H} \omega(x)}\right)$ e $y \in H$. Se $f_{n} \rightarrow 0$ uniformemente em $H$ então $\Omega(H)=\overline{\bigcup_{x \in H} \omega(x)}$.

\section{Prova:}

Como $\omega(x) \subset \Omega(H), \bigcup_{x \in H} \omega(x) \subset \Omega(H)$ e conseqüentemente $\overline{\bigcup_{x \in H} \omega(x)} \subset \overline{\Omega(H)}=\Omega(H)$ pois $\Omega(H)$ é fechado. Observe que não precisamos aqui da hipótese $f_{n} \rightarrow 0$ uniformemente em $H$.

Mostremos agora a outra inclusão. Como $f_{n} \rightarrow 0$ uniformemente em $H$ quando $n \rightarrow \infty$, então dado $\varepsilon>0$ existe $n_{0}=n_{0}(\varepsilon) \in \mathbb{N}$ tal que para todo $n \geq n_{0}$ temos que $f_{n}(y)<\varepsilon$, para todo $y \in H, \operatorname{assim} \sup _{y \in H} f_{n}(y) \leq \varepsilon \Rightarrow \sup _{y \in H} \rho\left(T^{n}(y), \overline{\bigcup_{x \in H} \omega(x)}\right) \leq \varepsilon \Rightarrow \sup _{z \in T^{n}(H)} \rho\left(z, \overline{\bigcup_{x \in H} \omega(x)}\right) \leq \varepsilon$.

Logo $\rho\left(T^{n}(H), \overline{\left.\bigcup_{x \in H} \omega(x)\right)} \rightarrow 0\right.$ quando $n \rightarrow \infty$ e portanto $T^{n}(H)$ se aproxima de $\overline{\bigcup_{x \in H} \omega(x)}$ quando $n \rightarrow \infty$. Como $\Omega(H)$ é o menor fechado do qual $T^{n}(H)$ se aproxima quando $n \rightarrow \infty$, temos que $\Omega(H) \subset \overline{\bigcup_{x \in H} \omega(x)}$.

Proposição 1.4.3. Se $K \subset \mathbb{R}^{m}$ é compacto não vazio e positivamente invariante, então $\Omega(K)=$ $\bigcap_{n=0}^{\infty} T^{n}(K)$. Além disso, $\Omega(K)$ é não vazio, compacto e é o maior conjunto invariante contido em $K$.

\section{Prova:}

Como $K$ é positivamente invariante, temos que $T^{n}(K) \subset K$ para todo n, e como $K$ também é compacto, pelo Teorema 1.4.2, $\Omega(K)$ é não vazio, compacto e invariante.

Temos que $T^{n}(K) \subset T^{j}(K)$ se $n \geq j$. Segue que $\overline{\bigcup_{n=j}^{\infty} T^{n}(K)} \subset \overline{T^{j}(K)}=T^{j}(K)$, pois $T$ é contínua. Assim $\Omega(K)=\bigcap_{j=0}^{\infty} \overline{\bigcup_{n=j}^{\infty} T^{n}(K)} \subset \overline{\bigcap_{j=0}^{\infty} T^{j}(K)}=\bigcap_{j=0}^{\infty} T^{j}(K)$ pelo teorema dos compactos encaixados, portanto $\Omega(K) \subset \bigcap_{j=0}^{\infty} T^{j}(K)$. 
Por outro lado, como $T^{j}(K) \subset \bigcup_{n=j}^{\infty} T^{n}(K) \subset \overline{\bigcup_{n=j}^{\infty} T^{n}(K)}$ para todo $j \in \mathbb{N}$, portanto $\bigcap_{j=0}^{\infty} T^{j}(K) \subset$ $\bigcap_{j=0}^{\infty} \overline{\bigcup_{n=j}^{\infty} T^{n}(K)}=\Omega(K) . \operatorname{Logo} \Omega(K)=\bigcap_{j=0}^{\infty} T^{j}(K)$.

Se $W \subset K$ é invariante, temos que $W=T^{n}(W) \subset T^{n}(K)$ para todo $n \in \mathbb{N}$. Assim $W \subset$ $\bigcap_{n=0}^{\infty} T^{n}(K)=\Omega(K)$. Logo $\Omega(K)$ é invariante maximal. 


\section{Estabilidade e instabilidade}

\section{$\mathrm{S}_{\text {eja } H \subset \mathbb{R}^{m} T \text {-invariante; }}$}

1. $H$ é localmente atrativo (ou atrator local) se existe uma vizinhança $W$ de $H$ ( $W$ é um conjunto aberto que contém $\bar{H}$ ) tal que $H$ atrai os pontos de $W$. Isto é, para todo $x \in W$ temos que $\lim _{n \rightarrow \infty} d\left(T^{n}(x), H\right)=0$.

2. $H$ é estável se para qualquer vizinhança $V$ de $H$ existe uma vizinhança $W$ de $H$ tal que $T^{n}(W) \subset V$ para todo $n \in \mathbb{N}$. Um conjunto que não é estável, é dito instável.

3. $H$ é localmente assintoticamente estável se for estável e existir uma vizinhança $W$ de $H$ tal que $\lim _{n \rightarrow \infty} d\left(T^{n}(x), H\right)=0$ para todo $x \in W$. Se H for estável e $\lim _{n \rightarrow \infty} d\left(T^{n}(x), H\right)=0$ para todo $x \in \mathbb{R}^{m}$, então $H$ é dito globalmente assintoticamente estável.

4. $H$ é uniformemente assintoticamente estável se for estável e atrair uma vizinhança $W$ de $H$.

\subsection{Método direto de Liapunov}

Proposição 2.1.1. Se H é estável, então $\bar{H}$ é estável e positivamente invariante. Em particular, se um ponto é estável, então ele é um ponto de equilíbrio.

\section{Prova:}

Se $H$ é estável, então dada uma vizinhança $V$ de $H$, existe uma vizinhança $W$ de $H$ tal que $T(W) \subset V$ e essas mesmas vizinhanças mostram a estabilidade de $\bar{H}$.

Para mostrar que $\bar{H}$ é positivamente invariante, considere as vizinhanças $U_{i}=B_{\frac{1}{i}}(H), i \in$ $\mathbb{N}-\{0\}$. Temos que $\bar{H}=\bigcap_{i=1}^{\infty} U_{i}$. 
De fato, se $x \in \bigcap_{i=1}^{\infty} U_{i}$, então $\rho(x, H)<\frac{1}{i}$ para todo $i \in \mathbb{N}-\{0\}, \log \rho \rho(x, H)=0$ e conseqüentemente, $x \in \bar{H}$, portanto $\bigcap_{i=1}^{\infty} U_{i} \subseteq \bar{H}$.

Por outro lado, como $\bar{H} \subset U_{i}$ para cada $i \in \mathbb{N}-\{0\}$, temos que $\bar{H} \subseteq \bigcap_{i=1}^{\infty} U_{i}, \log \mathrm{o} \bar{H}=\bigcap_{i=1}^{\infty} U_{i}$.

Como $\bar{H}$ é estável, para cada vizinhança $U_{i}$ existe uma vizinhança $W_{i}$ de $H$ tal que $T^{n}\left(W_{i}\right) \subset$ $U_{i}$ para todo $n, i \in \mathbb{N}-\{0\}$.

Como $W_{i} \subset U_{i}$, temos $\bigcap_{i=1}^{\infty} W_{i} \subset \bigcap_{i=1}^{\infty} U_{i}=\bar{H}, \log \bigcap_{i=1}^{\infty} W_{i}=\bar{H}$, e como $\bar{H} \subset W_{i}$ para cada $i \in \mathbb{N}-\{0\}$, temos que $\bar{H} \subset \bigcap_{i=1}^{\infty} W_{i}, \operatorname{logo} \bar{H}=\bigcap_{i=1}^{\infty} W_{i}$.

Finalmente, $T(\bar{H})=T\left(\bigcap_{i=1}^{\infty} W_{i}\right) \subset \bigcap_{i=1}^{\infty} T\left(W_{i}\right) \subset \bigcap_{i=1}^{\infty} U_{i}=\bar{H}$, logo $T(\bar{H}) \subset \bar{H}$.

Se $H=\left\{x_{0}\right\}$, onde $x_{0}$ é um ponto estável, temos que $\bar{H}=\left\{x_{0}\right\}=H$ e que $\bar{H}$ é positivamente invariante, isto é, $T(\bar{H}) \subset \bar{H}$. Como esse conjunto é unitário, obtemos que $T(\bar{H})=\bar{H}$, donde $x_{0}$ é um ponto de equilíbrio.

Definição 2.1.1. Um ponto de equilíbrio $\bar{x}$ de (1.1) é estável no sentido de Liapunov se para todo $\varepsilon>0$ existe um $\delta>0$ tal que $T^{n}\left(B_{\delta}(\bar{x})\right) \subset B_{\varepsilon}(\bar{x})$ para todo $n \in \mathbb{N}$, isto é, $\left\|T^{n}(x)-\bar{x}\right\|<\varepsilon$. Além disso, se para todo $x \in B_{\delta}(\bar{x})$ tivermos que $\lim _{n \rightarrow \infty} T^{n}(x)=\bar{x}$, dizemos que $\bar{x}$ é assintóticamente estável.

Se um ponto de equilíbrio não é estável, então ele é instável.

Definição 2.1.2. Um funcional $V: \mathbb{R}^{m} \rightarrow \mathbb{R}$ é dito definido positivo com respeito a $\bar{x}$ se:

1. $V(\bar{x})=0$.

2. Existe $\eta>0$ tal que $V(x)>0$ para qualquer $x \in B_{\eta}(\bar{x})-\{\bar{x}\}$.

Definição 2.1.3. Seja $V: \mathbb{R}^{m} \rightarrow \mathbb{R}$, define-se a "derivada" discreta de $V$ com respeito a $T$ como sendo $\dot{V}(x):=V(T(x))-V(x)$.

Definição 2.1.4. Seja $G \subset \mathbb{R}^{m}$, dizemos que $V$ é uma função de Liapunov para o sistema em $G$ se:

1. Vé contínua em $\mathbb{R}^{m}$.

2. $\dot{V}(x) \leq 0$ para todo $x \in G$.

Proposição 2.1.2 (Teorema de estabilidade de Liapunov).

Se $\bar{x}$ é um ponto de equilíbrio de $T, V$ é uma função de Liapunov para $T$ em alguma vizinhança de $\bar{x}$ e $V$ é definida positiva com respeito a $\bar{x}$, então $\bar{x}$ é um ponto de equilíbrio estável. 


\section{Prova:}

Tome $\eta$ suficientemente pequeno de modo que $V(x)>0$ e $\dot{V}(x) \leq 0$ para todo $x$ em $B_{\eta}(\bar{x})$ e seja $0<\varepsilon<\eta$.

Seja $m:=\min \{V(x):\||x-\bar{x} \||=\varepsilon\}$. Temos que $m$ é positivo.

Seja $G=\left\{x: V(x)<\frac{m}{2}\right\}$ e $G_{0}$ a componente conexa de $G$ que contém $\bar{x}$. Temos que tanto $G$ quanto $G_{0}$ são abertos.

Se $x_{0} \in G$, então $\dot{V}\left(x_{0}\right) \leq 0$, assim, $V\left(T\left(x_{0}\right)\right) \leq V\left(x_{0}\right)<\frac{m}{2}$, logo $T\left(x_{0}\right) \in G$.

$x_{0}$ e $\bar{x}$ estão na mesma componente conexa de $G$, e $T(\bar{x})=\bar{x}$ e $T\left(x_{0}\right)$ também estão nessa componente. Assim $G_{0}$ é um conjunto aberto e positivamente invariante contendo $\bar{x}$ e contido em $B_{\varepsilon}(\bar{x})$.

Como $V$ é contínua, existe $\delta>0$ tal que $B_{\delta}(\bar{x}) \subset G_{0}$. Assim, se $x_{0} \in B_{\delta}(\bar{x})$, então $x_{0} \in G$ e $T\left(x_{0}\right) \subset G_{0} \subset B_{\varepsilon}(\bar{x})$.

Proposição 2.1.3. Se V é uma função de Liapunov para (1.1) no conjunto, onde $|x|>N e$ $V(x) \rightarrow \infty$ quando $|x| \rightarrow \infty$, então todas as soluções desse sistema são limitadas.

\section{Prova:}

Suponha que exista $x_{0}$ tal que $T^{n}\left(x_{0}\right) \rightarrow \infty$ quando $n \rightarrow \infty$.

Como $V$ é uma função de Liapunov, temos que $0 \geq \dot{V}\left(x_{0}\right)=V\left(T\left(x_{0}\right)\right)-V\left(x_{0}\right)$, logo $0 \leq$ $V\left(T\left(x_{0}\right)\right) \leq V\left(x_{0}\right)$ e via indução finita, $0 \leq \cdots V\left(T^{n}\left(x_{0}\right)\right) \leq \cdots \leq V\left(T^{2}\left(x_{0}\right)\right) \leq V\left(T\left(x_{0}\right)\right) \leq$ $V\left(x_{0}\right)$. Absurdo pois $V(x) \rightarrow \infty$ quando $|x| \rightarrow \infty$.

J. P. LaSalle estabeleceu uma relação entre funções de Liapunov e conjuntos $\omega$-limites de Birkhoff, desenvolvendo o Princípio de invariância de LaSalle (Veja LaSalle, [18]).

Teorema 2.1.1 (Princípio de invariância de LaSalle).

Se $V$ é uma função de Liapunov para (1.1) em $G$ e se a solução $T^{n}\left(x_{0}\right)$ está em $G$ e é limitada, então existe um número c tal que $T^{n}\left(x_{0}\right) \rightarrow M \cap V^{-1}(c)$, onde $M$ é o maior conjunto positivamente invariante contido no conjunto $E=\left\{x \in \mathbb{R}^{m}: \dot{V}(x)=0\right\} \cap \bar{G}^{1}$.

\section{Prova:}

Como $x_{n}=T^{n}\left(x_{0}\right)$ está em $G$ e é limitada, pelo Teorema 1.4.1, temos que $\varnothing \neq \omega\left(x_{0}\right) \subset \bar{G}$ e $x_{n}$ tende para $\omega\left(x_{0}\right)$.

Como $V$ é uma função de Liapunov, temos $\cdots V\left(x_{n}\right) \leq \cdots \leq V\left(x_{2}\right) \leq V\left(x_{1}\right) \leq V\left(x_{0}\right)$, isto é, $V\left(x_{n}\right)$ é uma seqüência monótona não crescente e como $V$ é limitada inferiormente, temos que $V\left(x_{n}\right) \rightarrow c$ quando $n \rightarrow \infty$.

\footnotetext{
${ }^{1} \mathrm{O}$ conjunto E descrito no Teorema 2.1.1 é dito ser um conjunto de LaSalle
} 


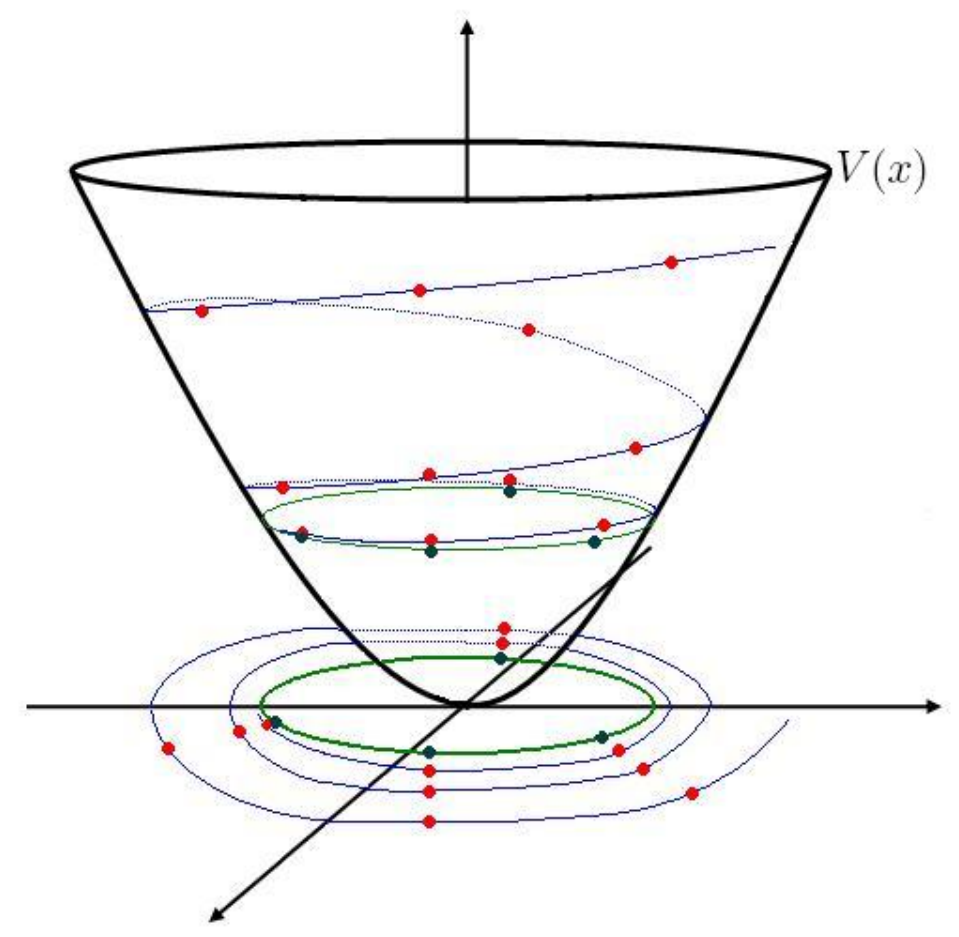

Figura 2.1: Ilustração do princípio de invariância de LaSalle

Se $y \in \omega\left(x_{0}\right)$ existe uma subseqüência $x_{n_{i}}$ tal que $x_{n_{i}} \rightarrow y$. Assim, $V\left(x_{n_{i}}\right) \rightarrow V(y)=c$, logo $V\left(\omega\left(x_{0}\right)\right)=c$, conseqüentemente, $\omega\left(x_{0}\right) \subset V^{-1}(c)$.

Como $V\left(\omega\left(x_{0}\right)\right)=c$ e $\omega\left(x_{0}\right)$ é positivamente invariante, $\dot{V}\left(\omega\left(x_{0}\right)\right)=0$, assim, $x_{n} \rightarrow \omega\left(x_{0}\right) \subset$ $\left\{x \in \mathbb{R}^{m}: \dot{V}(x)=0\right\} \cap \bar{G} \cap V^{-1}(c)$.

Como $\omega\left(x_{0}\right)$ é positivamente invariante, temos que $\omega\left(x_{0}\right) \subset M$.

O princípio de invariância de LaSalle nos permite estudar a estabilidade das soluções de uma equação de diferenças, não sendo necessário o conhecimento prévio das soluções, para isso necessitamos de uma função auxiliar $V$, denominada função de Liapunov. O princípio de invariância de LaSalle no entanto não nos fornece um método para encontrar uma função de Liapunov e, se o sistema dado pela equação de diferenças for complexo, isto é, suas trajetórias têm comportamento caótico ou tendem para atratores estranhos por exemplo, dificilmente encontraremos uma função de Liapunov para esse sistema. Na próxima seção veremos uma versão mais geral do princípio de invariância, na qual não se exige que a derivada discreta da função de Liapunov seja sempre não crescente ao longo das soluções.

Como conseqüência do Teorema de estabilidade de Liapunov e do Princípio de invariância de LaSalle, temos o Teorema de estabilidade assintótica de Liapunov (Veja LaSalle, [18]).

Corolário 2.1.1 (Teorema de estabilidade assintótica de Liapunov). 
Se $V e-\dot{V}$ são positivas definidas com respeito a $\bar{x}$, então $\bar{x}$ é assintoticamente estável.

\section{Prova:}

Como $V$ e $-\dot{V}$ são definidas positivas com respeito a $\bar{x}$, existe $\eta>0$ tal que $V(x)>0$ e $-\dot{V}(x)>0$ para todo $x \in B_{\eta}(\bar{x})-\bar{x}$.

Pelo Teorema de estabilidade de Liapunov temos que $\bar{x}$ é um ponto de equilíbrio estável, e pelo Princípio de invariância de LaSalle temos que se $x_{0} \in B_{\eta}(\bar{x})$ então $T^{n}\left(x_{0}\right) \rightarrow \bar{x}$, logo $\bar{x}$ é assintoticamente estável.

Proposição 2.1.4. Sejam $T: \mathbb{R}^{m} \rightarrow \mathbb{R}^{m}$ e $V: \mathbb{R}^{m} \rightarrow \mathbb{R}$ contínuas. Dado $L \in \mathbb{R}$ tome $\xi_{L}:=\{x \in$ $\left.\mathbb{R}^{m}: V(x)<L\right\}$. Suponha que $\dot{V}(x) \leq 0$ para todo $x \in \xi_{L}$. Então $\xi_{L}$ é positivamente invariante. Além disso, se $\xi_{L}$ é limitado, $E:=\left\{x \in \omega_{L}: \dot{V}(x)=0\right\}$ e $M$ é o maior conjunto invariante contido em $E$, então toda solução iniciando em $\xi_{L}$ converge para $M$ quando $n \rightarrow \infty$.

\section{Prova:}

Seja $x \in \xi_{L}$, temos que $\dot{V}(x)=V(T(x))-V(x) \leq 0, \log 0 V(T(x)) \leq V(x)<L$ e portanto $V(T(x)) \in \xi_{L}$. Segue que $T^{n}(x) \in \xi_{L}$ para todo $n \in \mathbb{N}$.

Se $\xi_{L}$ é limitado, temos que $T^{n}(x)$ é limitada, logo pelo Teorema 2.1.1, temos que toda solução iniciando em $\xi_{L}$ converge para $M$ quando $n \rightarrow \infty$.

Apresentaremos agora dois resultados sobre instabilidade (Veja LaSalle, [18], ou [19]).

Proposição 2.1.5. Seja $\dot{V}$ definida positiva com respeito a $\bar{x}$ e seja $V$ assumindo valores positivos arbitrariamente próximos de $\bar{x}$, então $\bar{x}$ é instável.

\section{Prova:}

Assuma que $\bar{x}$ é estável. Seja $\varepsilon>0$ suficientemente pequeno de modo que $\dot{V}(x)>0$ se $x \in B_{\varepsilon}(\bar{x})-\{\bar{x}\}$ e seja $\delta>0$ tal que se $x \in B_{\delta}(\bar{x})$ então $x_{n}=T^{n}(x) \in B_{\varepsilon}(\bar{x})$ para todo $n$.

Por hipótese existe $x_{0} \in B_{\delta}(\bar{x})$ tal que $V\left(x_{0}\right)>0$. Como $x_{n} \in B_{\varepsilon}(\bar{x})$, para todo $n$, pelo Princípio de invariância de LaSalle, temos que $x_{n}$ tende para $\left\{x \in \mathbb{R}^{m}: \dot{V}(x)=0\right\} \cap B_{\varepsilon}(\bar{x})=\{\bar{x}\}$.

Segue que $x_{n} \rightarrow \bar{x}$, conseqüentemente, $V\left(x_{n}\right) \rightarrow V(\bar{x})=0$. Mas $\dot{V}\left(x_{n}\right)>0$ assim, $V\left(T\left(x_{n}\right)\right)>$ $V\left(x_{n}\right)$ e portanto $V\left(x_{n-1}\right)>\cdots>V\left(x_{0}\right)>0$. Contradição.

Proposição 2.1.6. Assuma que $V$ toma valores positivos arbitrariamente próximos de $\bar{x}$. e $\dot{V}=\beta V+W$, onde $W(x) \geq 0$ em alguma vizinhança de $\bar{x} e \beta>1$. Então a origem é instável.

\section{Prova:}


Suponha que $\bar{x}$ seja estável e seja $\varepsilon>0$ suficientemente pequeno que $\dot{V}(x)>0$ para $x \in$ $B_{\varepsilon}(\bar{x})-\{\bar{x}\}$ e $\delta>0$ tal que se $x \in B_{\delta}(\bar{x})$ então $x_{n}=T^{n}(x) \in B_{\varepsilon}(\bar{x})$ para todo $\mathrm{n}$.

Por hipótese, existe um ponto $x_{0} \in B_{\delta}(\bar{x})$ tal que $V\left(x_{0}\right)>0$. Como $\left(x_{n}\right)$ é limitada e permanece em $B_{\varepsilon}(\bar{x}), x_{n}$ tende para $\bar{x}=\left\{x \in \mathbb{R}^{m}: \dot{V}(x)=0\right\} \cap B_{\varepsilon}(\bar{x})$, pelo Princípio de invariância.

Como $x_{n} \rightarrow \bar{x}$, temos que $V\left(x_{n}\right) \rightarrow V(\bar{x})=0$. Mas $\dot{V}\left(x_{n}\right)>0$, assim $V\left(x_{n}\right) \geq 0$, e assim $V\left(x_{n}\right) \geq V\left(x_{n-1}\right) \geq \cdots V\left(x_{0}\right)>0$. Essa contradição prova a proposição.

\subsection{Versão estendida do princípio de invariância}

Seja $\Lambda$ um subconjunto compacto de um espaço de Banach $E$. Nessa seção estudaremos equações da forma:

$$
x^{\prime}=T(x(n), n, \lambda)=T_{\lambda}(x(n), n),
$$

onde $\lambda \in \Lambda$ e $T: \mathbb{R}^{m} \times \mathbb{Z} \times \Lambda \rightarrow \mathbb{R}^{m}$ é contínua. Uma solução de (2.1) é uma sequiência $x(n, \lambda)$ que a satisfaz para todo $n \in \mathbb{N}$ e para todo $\lambda \in \Lambda$.

Dada $V: \mathbb{R}^{m} \times \mathbb{Z} \times \Lambda \rightarrow \mathbb{R}$, Para cada $(x, n, \lambda) \in \mathbb{R}^{m} \times \mathbb{Z} \times \Lambda$ definimos $\dot{V}(x, n, \lambda):=$ $V(T(x, n, \lambda), n, \lambda)-V(x, n, \lambda)$.

Na prática trabalhamos com funções de Liapunov generalizadas, cuja derivada discreta pode assumir valores positivos num conjunto limitado. Como veremos no Teorema.da dissipação uniforme, com isso podemos trabalhar com uma classe maior de problemas.

O próximo Teorema é um dos resultados mais importantes desse trabalho. Tendo sido desenvolvido por GABRIEL FILHO sob orientação de RODRIGUES, pode ser encontrado em RODRIGUES, WU, GABRIEL FILHO, [31] ou em GABRIEL FILHO, [8]. Um resultado similar pode ser encontrado em (ALBERTO, CALIERO, MARTINS, [2] ou CALIERO, [5]).

Teorema 2.2.1 (Teorema da dissipação uniforme).

Sejam $a, b: \mathbb{R}^{m} \mapsto \mathbb{R}$ funções contínuas tais que $0 \leq a(x) \leq V(x, n, \lambda) \leq b(x)$ para todo $(x, n, \lambda) \in \mathbb{R}^{m} \times \mathbb{Z} \times \Lambda$ e $a(x) \rightarrow \infty$ quando $|x| \rightarrow \infty$. Para todo $\rho>0$ defina $A_{\rho}:=\left\{x \in \mathbb{R}^{m}:\right.$ $a(x) \leq \rho\}$ e $B_{\rho}:=\left\{x \in \mathbb{R}^{m}: b(x) \leq \rho\right\}$.

Assuma que exista $H>0$ tal que para todo $\rho \in[0, H]$ o conjunto $A_{\rho}$ é conexo e assuma também que existe uma função contínua $c: A_{H} \rightarrow \mathbb{R}$ tal que $-\dot{V}(x, n, \lambda) \geq c(x)$, pra todo $(x, n, \lambda) \in A_{H} \times \mathbb{Z} \times \Lambda$, que o conjunto $\left\{x \in A_{H}: c(x)<0\right\} \neq \emptyset$ e que $\mathcal{C}:=\left\{x \in A_{H}: c(x) \leq 0\right\}$ é limitado. Finalmente assuma que existem constantes positivas $R$, $\mu$ e $H$ tais que $\max _{x \in C} b(x)<$ $R<\infty,-\mu<\min _{x \in C} c(x) e R+\mu<H$, então para cada $\lambda \in \Lambda$ as seguintes afirmações valem: 
1. Se $x_{0} \in B_{R}$ e se $x(n)$ é a solução de (2.1) com valor inicial $x_{0}$, então $x(n) \in A_{R+\mu}$ para todo $n \in \mathbb{N}$.

2. Se $x_{0} \in A_{H}$ então existe $n_{0}=n_{0}\left(x_{0}, \lambda\right) \geq 0$ tal que $x\left(n_{0}\right) \in B_{R}$ e $x(n) \in A_{R+\mu}$ para todo $n \geq n_{0}$. Em particular, se $\bar{x} \in A_{H}$ é um ponto de equilíbrio de $T_{\lambda}$, então $\bar{x} \in B_{R}$.

3. Se $x_{0} \in A_{H}$ e $x(n)$ é a solução de (2.1) com valor inicial $x(0)$, então existe uma subseqüência $n_{j}$ tal que $x\left(n_{j}\right) \in B_{R}$.

4. Se $x(n)$ satisfaz (2.1) para todo $n \in \mathbb{Z}$ e se $x(n) \in A_{H}$ para todo $n \in \mathbb{Z}$, então $x(n) \in A_{R+\mu}$ para todo $n \in \mathbb{Z}$. Em particular, toda solução periódica de (2.1) contida em $A_{H}$ está contida em $A_{R+\mu}$.

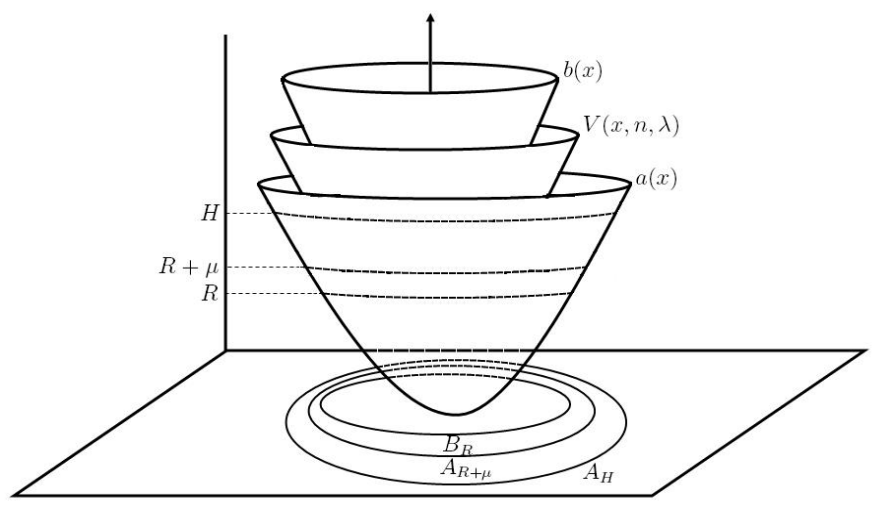

(a) Estimativas da função de Liapunov

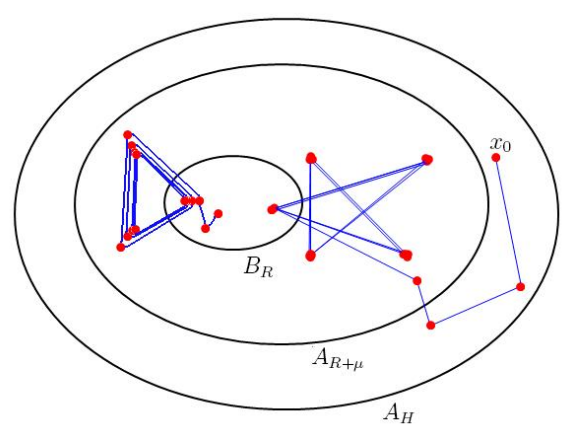

(b) Comportamento das soluções

Figura 2.2: Funções $a, V$ e $b$ do Teorema 2.2.1

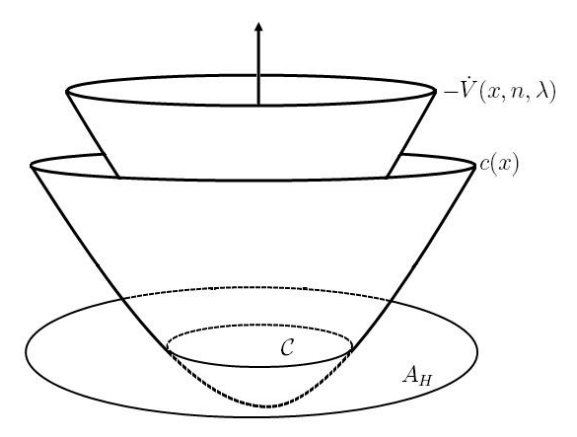

Figura 2.3: Estimativa da derivada discreta da função de Liapunov

\section{Prova:}


Sejam $T(., n)=T(., n, \lambda), V(., n)=V(., n, \lambda)$.

1)

Se $0 \leq \rho \leq H$ então $B_{\rho} \subset V_{\rho} \subset A_{\rho}$; onde $V_{\rho}:=\left\{x \in A_{H}: V(x, n) \leq \rho\right\}$. Temos também que $C \varsubsetneqq B_{R}$

Se $R+\mu \leq \rho \leq H$ então $V_{\rho}$ é positivamente invariante com respeito a (2.1). De fato, observe que se $x_{0} \in B_{R}$, então $-\dot{V}(x(0), 0) \geq c\left(x_{0}\right) \geq-\mu$ e assim, $V(T(x(0)))-V(x(0))=V(x(1))-$ $V(x(0))=\dot{V}(x(0)) \leq \mu$ e portanto $V(x(1)) \leq V(x(0))+\mu \leq b\left(x_{0}\right)+\mu \leq R+\mu \leq \rho$.

Isso mostra que se $x_{0} \in B_{R}$ então $x(1) \in V_{\rho}$, noutras palavras, se $x(0) \in V_{\rho}-B_{R}$, então $c(x(0))>0$ e assim $-V(T(x(0), 0)+V(x(0))=-\dot{V}(T(x(0), 0)) \geq c(x(0))>0$; seque que $V(x(1))=V(T(x(0), 0))<V(x(0)) \leq \rho$ e via indução finita, temos que $V_{\rho}$ é positivamente invariante.

Em particular, se $\rho=R+\mu$ e se $x(0) \in B_{R}$, então $x(n) \in V_{R+\mu} \subset A_{R+\mu}$ para todo $n \in \mathbb{Z}$.

2)

Assuma que se $x(0) \in A_{H}-B_{R}$ então existe $m=m(x(0), \lambda)$ tal que $x(m) \in B_{R}$.

Seja $\rho=\rho(x(0), \lambda):=\max \{V(x(0)), R+\mu\}$. Então o conjunto $V_{\rho}$ positivamente invariante. Seja $W:=V_{\rho}-B_{R}$. É fácil ver que se $x \in \bar{W}$ então $c(x)>0$. Seja $\beta:=\min _{x \in \bar{W}} c(x)$. Como $x(0) \in \bar{W}$, temos $c(x(0)>0$. Então $-[V(T(x(0))-V(x(0))]=-\dot{V}(x(0), 0) \geq c(x(0) \geq \beta$ ou $V(T(x(0)))-V(x(0)) \leq-\beta \mathrm{e} \operatorname{assim} V(x(1))=V(T(x(0), 0)) \leq V(x(0))-\beta$.

Se $x(1) \in B_{R}$ acabou, mas se $x(1) \in V_{\rho}-B_{R}$, então repetindo o processo acima, obtemos $V(x(2)) \leq V(x(1))-\beta \leq V(x(0))-2 \beta$.

Se $x(2) \in B_{R}$ acabou, se não, continuando com esse processo, após $n-1$ passagens temos $V(x(n) \leq V(x(0))-n \beta$.

Isso Mostra que existe $m \in \mathbb{N}$ tal que $x(m) \in B_{R}$, senão teríamos uma contradição pois $V(x) \geq 0$. Isso prova a afirmação.

Se $\bar{x} \in A_{H}$ é um ponto de equilíbrio de $T_{\lambda}$, temos que $\bar{x}=T_{\lambda}^{n}(\bar{x})$ para todo $n \in \mathbb{N}$ e pelo que vimos acima, existe $n_{0}=n_{0}(\bar{x}, \lambda)$ tal que $x\left(n_{0}\right)=T_{\lambda}^{n_{0}}(\bar{x})=\bar{x} \in B_{R}$, como queríamos.

Conseqüência da afirmação acima.

4)

Suponha que existe $m \in \mathbb{Z}$ tal que $x(m) \in A_{H}-V_{R+\mu}$. Seja $\kappa:=\min \left\{C(x): x \in \overline{A_{H}-V_{R+\mu}}\right\}$, 
é claro que $v>0$.

De II) segue que $x(m-1) \in A_{H}-V_{R+\mu}$. Então $-[V(x(m)-V(x(m-1))]=-\dot{V}(T(x(m-$ $1), m-1) \geq c(x(m-1)) \geq \kappa$.

Isso implica que $V(x(m))+\kappa V(x(m-1))$. Como $x(m-2) \in A_{H}-V_{R+\mu}$, repetindo o processo acima, obtemos $V(x(m))+2 \kappa \leq V(x(m-n)) \leq b(x(m-n))$. Isso é uma contradição, pois $b$ é uma função limitada em $A_{H}$.

Se $x(n)=T_{\lambda}^{n}(x)$ é uma órbita periódica contida em $A_{H}$, pela proposição $1.3 .5, x(n)$ admite um prolongamento em $A_{H}$ para todo $n \in \mathbb{Z}$. Assim, $x(n)$ satisfaz (2.1) para todo $n \in \mathbb{Z}$ e como vimos, $x(n) \in A_{R+\mu}$ para todo $n \in \mathbb{Z}$.

Se $\bar{x} \in A_{H}$ é um ponto de equilíbrio de $T_{\lambda}$, temos que $\bar{x}=T_{\lambda}^{n}(\bar{x})$ para todo $n \in \mathbb{N}$ e pelo que vimos acima, existe $n_{0}=n_{0}(\bar{x}, \lambda)$ tal que $x\left(n_{0}\right)=T_{\lambda}^{n_{0}}(\bar{x})=\bar{x} \in B_{R}$, como queríamos.

Teorema 2.2.2 (Versão estendida do Princípio de invariância).

Sejam $T: \mathbb{R}^{m} \times \Lambda \rightarrow \mathbb{R}^{m} ; V: \mathbb{R}^{m} \times \Lambda \rightarrow \mathbb{R}_{+}$contínuas e suponha que existam funções $a, c: \mathbb{R}^{m} \rightarrow \mathbb{R}$ satisfazendo $0 \leq a(x) \leq V(x, \lambda) e-\dot{V}(x, \lambda) \geq c(x) \geq 0$ para todo $(x, \lambda) \in \mathbb{R}^{m} \times \Lambda$.

Assuma que para todo $H>0$ o conjunto $A_{H}:=\left\{x \in \mathbb{R}^{m}: a(x) \leq H\right\}$ é limitado (isso acontece por exemplo se $a(x) \rightarrow \infty$ quando $|x| \rightarrow \infty)$ e seja $\left(x_{0}, \lambda_{0}\right) \in \mathbb{R}^{m} \times \Lambda$.

Então $\varepsilon_{c}:=\left\{x \in \mathbb{R}^{m}: c(x)=0\right\} \neq \varnothing$ e $T^{n}\left(x_{0}, \lambda_{0}\right)$ tende quando $n \rightarrow \infty$ para o maior conjunto invariante contido em $\varepsilon_{c}$.

\section{Prova:}

Sejam $T(., n)=T(., n, \lambda), V(., n)=V(., n, \lambda)$, uma vez fixado $\lambda$.

Po hipótese, $-\dot{V}(x) \geq c(x) \geq 0 \forall x \in \mathbb{R}^{m}$, $\log 0 \dot{V}(x) \leq(0) \forall x \in \mathbb{R}^{m}$ e conseqüentemente $\dot{V}(x)=V(T(x))-V(x) \leq 0 \Rightarrow V(T(x)) \leq V(x)$, e via indução finita:

$\cdots \leq V\left(T^{n}(x)\right) \leq \cdots \leq V\left(T^{2}(x)\right) \leq V(T(x)) \leq V(x)=: \rho$.

$V\left(T^{n}(x)\right)$ é uma seqüência monótona num conjunto limitado, e portanto convergente, digamos $V\left(T^{n}(x)\right) \rightarrow c$ quando $n \rightarrow \infty$.

Como $T^{n}(x)$ está contida num compacto, pelo Teorema 1.4.1, $\omega(x)$ é não vazio, e se $y \in$ $\omega(x)$ existe subsequiência $T^{n_{k}}(x) \rightarrow y$. Também pelo Teorema 1.4.1 $\omega(x)$ é invariante, portanto $T(y) \in \omega(x)$ e $T^{n_{k}+1}(x) \rightarrow T(y)$.

Temos que $c=\lim _{n \rightarrow \infty} V\left(T^{n_{k}}(x)\right)=V(y)$ e $c=\lim _{n \rightarrow \infty} V\left(T^{n_{k}+1}(x)\right)=V(T(y)), \log _{0} \dot{V}(y)=0$ e visto que $0 \leq c(y) \leq-\dot{V}(y)$ temos que $c(y)=0, \log 0 \varepsilon_{c} \neq \varnothing$ e $\omega(x) \subset \varepsilon_{c}$.

Finalmente, pelo princípio de invariância de LaSalle, concluímos que $T^{n}(x)$ converge para o maior conjunto invariante contido em $\varepsilon_{c}$. 


\subsection{Simulações e exemplos}

Nessa seção, apresentaremos alguns exemplos no qual o Teorema de dissipação uniforme é aplicável.

Exemplo 2.3.1 (Lorenz discreto $I$ ).

O seguinte sistema contínuo é uma variação do sistema de Lorenz e foi estudado por RODRIGUES e GAMEIRO, (veja [30]), na qual se propunha uma análise em sistemas de comunicação.

$$
\left\{\begin{array}{l}
\dot{x}(t)=-a x(t)+a y(t) \\
\dot{y}(t)=-y(t)-\frac{r}{4}(x(t)+\alpha(t))-(x(t)+\alpha(t)) z(t) \\
\dot{z}(t)=-b z(t)+(x+\alpha(t))-\frac{5}{4} b r
\end{array}\right.
$$

$\alpha$ é o sinal a ser transmitido e os parâmetros $a, b$, e r são tais a manter o comportamento caótico do sistema para garantir a segurança na comunicação.

Considere o sistema obtido pela discretização do sistema de Lorenz (via método iterativo de Euler), $a \in\left[a_{m}, a_{M}\right] ; r \in\left[r_{m}, r_{M}\right] ; b \in\left[b_{m}, b_{M}\right] ; h \in \mathbb{R}_{+}, \alpha(n)=\alpha_{n} \in l_{\infty} e \lambda=\left(a, b, r,\left(\alpha_{n}\right), h\right)$ :

$$
\left\{\begin{array}{l}
x(n+1)=x(n)+h[-a x(n)+a y(n)] \\
y(n+1)=y(n)+h\left[-y(n)-\frac{r}{4}\left(x(n)+\alpha_{n}\right)-\left(x(n)+\alpha_{n}\right) z(n)\right] \\
z(n+1)=z(n)+h\left[-b z(n)+\left(x(n)+\alpha_{n}\right) y(n)-\frac{5}{4} b r\right]
\end{array}\right.
$$

Considere a função de Liapunov generalizada $V(x, y, z, \lambda)=r x^{2}+4 a y^{2}+4 a z^{2}$ para esse sistema. É fácil tomar funções a e b como no teorema de dissipação uniforme, observando que as seguintes desigualdades são válidas:

$$
a(x, y, z):=r_{m} x^{2}+4 a_{m} y^{2}+4 a_{m} z^{2} \leq V(x, y, z, \lambda) \leq r_{M} x^{2}+4 a_{M} y^{2}+4 a_{M} z^{2}=: b(x, y, z) .
$$

A escolha da função c não é tão imediata. Calculemos inicialmente $-\dot{V}$ :

$$
\begin{aligned}
& -\dot{V}(x, y, z, n, \lambda)=r x^{2}+4 a y^{2}+4 a z^{2}-\left\{r\{x+h[-a x+a y]\}^{2}+4 a\left\{y+h\left[-y-\frac{r}{4}\left(x+\alpha_{n}\right)-(x+\right.\right.\right. \\
& \left.\left.\left.\left.\alpha_{n}\right) z\right]\right\}^{2}+4 a\left\{z+h\left[-b z+\left(x+\alpha_{n}\right) y-\frac{5}{4} b r\right]\right\}^{2}\right\}= \\
& r x^{2}+4 a y^{2}+4 a z^{2}-r\{x+h[-a x+a y]\}^{2}-4 a\left\{y+h\left[-y-\frac{r}{4}\left(x+\alpha_{n}\right)-\left(x+\alpha_{n}\right) z\right]\right\}^{2}-4 a\{z+ \\
& \left.h\left[-b z+\left(x+\alpha_{n}\right) y-\frac{5}{4} b r\right]\right\}^{2}= \\
& r x^{2}+4 a y^{2}+4 a z^{2}-r\left\{x^{2}+2 h x[-a x+a y]+h^{2}[-a x+a y]^{2}\right\}-4 a\left\{y^{2}+2 h y\left[-y-\frac{r}{4}\left(x+\alpha_{n}\right)-\right.\right. \\
& \left.\left.\left(x+\alpha_{n}\right) z\right]+h^{2}\left[-y-\frac{r}{4}\left(x+\alpha_{n}\right)-\left(x+\alpha_{n}\right) z\right]^{2}\right\}-4 a\left\{z^{2}+2 h z\left[-b z+\left(x+\alpha_{n}\right) y-\frac{5}{4} b r\right]+h^{2}[-b z+\right. \\
& \left.\left.\left(x+\alpha_{n}\right) y-\frac{5}{4} b r\right]^{2}\right\}=
\end{aligned}
$$


$r x^{2}+4 a y^{2}+4 a z^{2}-r x^{2}-2 h r x[-a x+a y]-h^{2} r[-a x+a y]^{2}-4 a y^{2}-8 h a y\left[-y-\frac{r}{4}\left(x+\alpha_{n}\right)-(x+\right.$ $\left.\left.\alpha_{n}\right) z\right]-4 h^{2} a\left[-y-\frac{r}{4}\left(x+\alpha_{n}\right)-\left(x+\alpha_{n}\right) z\right]^{2}-4 a z^{2}-8 h a z\left[-b z+\left(x+\alpha_{n}\right) y-\frac{5}{4} b r\right]-4 h^{2} a[-b z+$ $\left.\left(x+\alpha_{n}\right) y-\frac{5}{4} b r\right]^{2}=$

$-2 h r x[-a x+a y]-h^{2} r[-a x+a y]^{2}-8 h a y\left[-y-\frac{r}{4}\left(x+\alpha_{n}\right)-\left(x+\alpha_{n}\right) z\right]-4 h^{2} a\left[-y-\frac{r}{4}\left(x+\alpha_{n}\right)-\right.$ $\left.\left(x+\alpha_{n}\right) z\right]^{2}-8 h a z\left[-b z+\left(x+\alpha_{n}\right) y-\frac{5}{4} b r\right]-4 h^{2} a\left[-b z+\left(x+\alpha_{n}\right) y-\frac{5}{4} b r\right]^{2}=$

$h\left\{-2 r x[-a x+a y]-8 a y\left[-y-\frac{r}{4}\left(x+\alpha_{n}\right)-\left(x+\alpha_{n}\right) z\right]-8 a z\left[-b z+\left(x+\alpha_{n}\right) y-\frac{5}{4} b r\right]\right\}+$ $h^{2}\left\{-r[-a x+a y]^{2}-4 a\left[-y-\frac{r}{4}\left(x+\alpha_{n}\right)-\left(x+\alpha_{n}\right) z\right]^{2}-4 a\left[-b z+\left(x+\alpha_{n}\right) y-\frac{5}{4} b r\right]^{2}\right\}=$

$h\left\{2 a r x^{2}-2 a r x y+8 a y^{2}+2 a r\left(x+\alpha_{n}\right) y+8 a\left(x+\alpha_{n}\right) y z+8 a b z^{2}-8 a\left(x+\alpha_{n}\right) y z+10 a b r z\right\}+$ $h^{2}\left\{-r[-a x-a y]^{2}-4 a\left[-y-\frac{r}{4}\left(x+\alpha_{n}\right)-\left(x+\alpha_{n}\right) z\right]^{2}-4 a\left[-b z+\left(x+\alpha_{n}\right) y-\frac{5}{4} b r\right]^{2}\right\}=$

$h\left\{2 a r x^{2}-2 a r x y+8 a y^{2}+2 a r x y+2 a \alpha_{n} r y+8 a x y z+8 a \alpha_{n} y z+8 a b z^{2}-8 a x y z-8 a \alpha_{n} y z+\right.$ $10 a b r z\}+h^{2}\left\{-r[-a x-a y]^{2}-4 a\left[-y-\frac{r}{4}\left(x+\alpha_{n}\right)-\left(x+\alpha_{n}\right) z\right]^{2}-4 a\left[-b z+\left(x+\alpha_{n}\right) y-\frac{5}{4} b r\right]^{2}\right\}=$ $h\left\{2 a r x^{2}+8 a y^{2}+8 a b z^{2}+2 a \alpha_{n} r y+10 a b r z\right\}+h^{2}\left\{-r[-a x-a y]^{2}-4 a\left[-y-\frac{r}{4}\left(x+\alpha_{n}\right)-(x+\right.\right.$ $\left.\left.\left.\alpha_{n}\right) z\right]^{2}-4 a\left[-b z+\left(x+\alpha_{n}\right) y-\frac{5}{4} b r\right]^{2}\right\}$

Assim, $-\frac{\dot{V}(x, y, z)}{h}=2 a r x^{2}+8 a y^{2}+8 a b z^{2}+2 a \alpha_{n} r y+10 a b r z+h g(x, y, z, n, \lambda) \geq 2 a_{m} r_{m} x^{2}+$ $8 a_{m} y^{2}+8 a_{m} b_{m} z^{2}-2 a_{M}\left|\alpha_{n}\right| r_{M}|y|-10 a_{M} b_{M} r_{M}|z|-h|g(x, y, z, n, \lambda)|$, onde assumiremos que $\left|\alpha_{n}\right| \leq \gamma$ para todo $n \in \mathbb{N}$.

Seja $d(x, y, z):=2 a_{m} r_{m} x^{2}+8 a_{m} y^{2}+8 a_{m} b_{m} z^{2}-2 a_{M} \gamma r_{M}|y|-10 a_{M} b_{M} r_{M}|z|$. Completando quadrados, temos $d(x, y, z)=2 a_{m} r_{m} x^{2}+8 a_{m}\left[y^{2}-2|y| \frac{a_{M} \gamma r_{M}}{8 a_{m}}+\left(\frac{a_{M} \gamma_{M}}{8 a_{m}}\right)^{2}\right]+$ $8 a_{m} b_{m}\left[z^{2}-2|z| \frac{5 a_{M} b_{M} r_{M}}{8 a_{m} b_{m}}+\left(\frac{5 a_{M} b_{M} r_{M}}{8 a_{m} b_{m}}\right)^{2}\right]-\frac{\left(a_{M} \gamma r_{M}\right)^{2}}{8 a_{m}}-\frac{\left(5 a_{M} b_{M} r_{M}\right)^{2}}{8 a_{m} b_{m}}=2 a_{m} r_{m} x^{2}+8 a_{m}[|y|-$ $\left.\frac{a_{M} \gamma_{M}}{8 a_{m}}\right]^{2}+8 a_{m} b_{m}\left[|z|-\frac{5 a_{M} b_{M} r_{M}}{8 a_{m} b_{m}}\right]^{2}-\frac{\left(a_{M} \gamma_{M}\right)^{2}}{8 a_{m}}-\frac{\left(5 a_{M} b_{M} r_{M}\right)^{2}}{8 a_{m} b_{m}}$.

Segue que o mínimo de dé $-\frac{\left(a_{M} \gamma r_{M}\right)^{2}}{8 a_{m}}-\frac{\left(5 a_{M} b_{M} r_{M}\right)^{2}}{8 a_{m} b_{m}}<0$.

Seja $\mathcal{D}:=\{(x, y, z): d(x, y, z) \leq 0\}$ e sejam $R_{1}$ e $R$ tais que $\max \{b(x, y, z) ;(x, y, z) \in \mathcal{D}\}<$ $R_{1}<R$. Escolheremos $H$ tão grande quanto $R$.

Dado $\delta>0$, existe $h_{0}>0$, suficientemente pequeno e tal que $h \max \{\mid g(x, y, z, n, \lambda) ;(x, y, z) \in$ $\left.A_{H} \mid\right\}<\delta$ se $0<h<h_{0}$.

Seque que se $(x, y, z) \in A_{H}$, então $d(x, y, z)-|h g(x, y, z)| \geq d(x, y, z)-\left(\frac{a_{M} \gamma_{M}}{8 a_{m}}\right)^{2}-$ $\left(\frac{5 a_{M} b_{M} r_{M}}{8 a_{m} b_{m}}\right)^{2}-\delta=: d_{1}(x, y, z)$.

Para $C:=\left\{(x, y, z): d_{1}(x, y, z) \leq 0\right\}$, podemos mostrar que se $\delta$ é suficientemente pequeno, então existe $h_{0}$ tal que $\max \{b(x, y, z):(x, y, z) \in \mathcal{C}\}<R$ para todo $0<h<h_{0}$. 
$\operatorname{Se} c(x, y, z):=h d_{1}(x, y, z)$ temos que $-\dot{V}(x, y, z, n, \lambda) \geq c(x, y, z), \forall(x, y, z) \in A_{H}$.

$O$ mínimo de c $(x, y, z)$ em $C$ é dado por $-h\left[\frac{\left(a_{M} r_{M} \gamma\right)^{2}}{8 a_{m}}+\frac{\left(5 a_{M} b_{M} r_{M}\right)^{2}}{8 a_{m} b_{m}}+\delta\right] \geq-h_{0}\left[\frac{\left(a_{M} r_{M} \gamma\right)^{2}}{8 a_{m}}+\right.$ $\left.\frac{\left(5 a_{M} b_{M} r_{M}\right)^{2}}{8 a_{m} b_{m}}+\delta\right]>-\mu$, para um $\mu>0$ apropriado, o qual pode ser tomado pequeno, se $h_{0}$ for suficientemente pequeno.

Tomando então ho suficientemente pequeno que $R+\mu<H$, então todas as condições do Teorema 2.2.1 estão satisfeitas para todo $0<h<h_{0}$ e assim, o atrator está contido em $A_{R+\mu}$.

É importante determinar o máximo de $b(x, y, z)$ em $\mathcal{D}$ que irá ocorrer na fronteira de $\mathcal{D}$, que é um elipsóide. Pelo método dos multiplicadores de Lagrange:

$$
\left\{\begin{array}{cc}
b_{x} & =\beta d_{x} \\
b_{y} & =\beta d_{y} \\
b_{z} & =\beta d_{z} \\
d(x, y, z) & =0
\end{array}\right.
$$

obtemos o sistema:

$$
\left\{\begin{array}{ccc}
2 r_{M} x & = & \beta 4 a_{m} r_{m} x \\
8 a_{M} y & = & \beta\left(16 a_{m} y-2 a_{M} \gamma_{M}\right) \\
8 a_{M} z & = & \beta\left(16 a_{m} b_{m} z-10 a_{M} b_{M} r_{M}\right) \\
2 a_{m} r_{m} x^{2}+8 a_{m}\left[|y|-\frac{a_{M} r_{M}}{8 a_{m}}\right]^{2}+8 a_{m} b_{m}\left[|z|-\frac{5 a_{M} b_{M} r_{M}}{8 a_{m} b_{m}}\right]^{2} & = & \frac{\left(a_{M} \gamma_{M}\right)^{2}}{8 a_{m}}+\frac{\left(5 a_{M} b_{M} r_{M}\right)^{2}}{8 a_{m} b_{m}}
\end{array}\right.
$$

De (1), (2) e de (3) obtemos respectivamente $x=0, y=\frac{\beta a_{M} \gamma r_{M}}{8 \beta a_{m}-4 a_{M}}$ e $z=\frac{5 \beta a_{M} b_{M} r_{M}}{8 \beta a_{m} b_{m}-4 a_{M}}$. Substuindo em (4):

$$
\begin{gathered}
8 a_{m}\left[\frac{\beta a_{M} \gamma_{M}}{8 \beta a_{m}-4 a_{M}}-\frac{a_{M} \gamma_{M}}{8 a_{m}}\right]^{2}+8 a_{m} b_{m}\left[\frac{5 \beta a_{M} b_{M} r_{M}}{8 \beta a_{m} b_{m}-4 a_{M}}-\frac{5 a_{M} b_{M} r_{M}}{8 a_{m} b_{m}}\right]^{2}=\frac{\left(a_{M} \gamma r_{M}\right)^{2}}{8 a_{m}}+\frac{\left(5 a_{M} b_{M} r_{M}\right)^{2}}{8 a_{m} b_{m}} \Rightarrow \\
8 a_{m}\left[\frac{2 \beta a_{M} a_{m} r_{M} \gamma-2 \beta a_{M} a_{m} r_{M} \gamma+a_{M}^{2} r_{M} \gamma}{16 \beta a_{m}^{2}-8 a_{M} a_{m}}\right]^{2}+8 a_{m} b_{m}\left[\frac{10 \beta a_{M} a_{m} b_{M} b_{m} r_{M}-10 \beta a_{M} a_{m} b_{M} b_{m} r_{M}+5 a_{M}^{2} b_{M} r_{M}}{16 \beta a_{m}^{2} b_{m}^{2}-8 a_{M} a_{m} b_{m}}\right]^{2}= \\
\frac{b_{m}\left(a_{M} \gamma r_{M}\right)^{2}+\left(5 a_{M} b_{M} r_{M}\right)^{2}}{8 a_{m} b_{m}} \Rightarrow \\
b_{m}\left[\frac{a_{M}^{2} r_{M} \gamma}{2 \beta a_{m}-a_{M}}\right]^{2}+\left[\frac{5 a_{M}^{2} b_{M} r_{M}}{2 \beta a_{m} b_{m}-a_{M}}\right]^{2}=\left(a_{M} r_{M}\right)^{2}\left[b_{m} \gamma^{2}+\left(5 b_{M}\right)^{2}\right] \Rightarrow \\
b_{m}\left[\frac{a_{M} \gamma}{2 \beta a_{m}-a_{M}}\right]^{2}+\left[\frac{5 a_{M} b_{M}}{2 \beta a_{m} b_{m}-a_{M}}\right]^{2}=\left[b_{m} \gamma^{2}+\left(5 b_{M}\right)^{2}\right] \Rightarrow \\
b_{m}\left(a_{M} \gamma\right)^{2}\left(2 \beta a_{m} b_{m}-a_{M}\right)^{2}+\left(5 a_{M} b_{M}\right)^{2}\left(2 \beta a_{m}-a_{M}\right)^{2}= \\
{\left[b_{m} \gamma^{2}+\left(5 b_{M}\right)^{2}\right]\left(2 \beta a_{m}-a_{M}\right)^{2}\left(2 \beta a_{m} b_{m}-a_{M}\right)^{2} \Rightarrow}
\end{gathered}
$$

Obtemos então uma equação polinomial de grau 4 em $\beta$, que foi resolvida numericamente, usando Mathematica, tomando $\gamma=4$ e supondo que os parâmetros a, $r$ e b valem 10, 28 e $\frac{8}{3}$ respectivamente com uma incerteza de $2 \%$. Temos então: 


$$
\begin{array}{ll}
a_{m}=a-\frac{2 a}{100}=\frac{49}{5}, & a_{M}=a+\frac{2 a}{100}=\frac{51}{5}, \\
r_{m}=r-\frac{2 r}{100}=\frac{686}{25}, & r_{M}=r+\frac{2 r}{100}=\frac{714}{25}, \\
b_{m}=b-\frac{2 b}{100}=\frac{196}{75}, & b_{M}=b+\frac{2 b}{100}=\frac{204}{75}
\end{array}
$$

Resolvendo essa equação, encontramos os valores $\beta=0, \beta=0.756499$ e mais duas raízes complexas não reais. $\beta=0$ dá o mínimo e $\beta=0.756499$ dá o máximo para a função $b$ no elipsóide $\{(x, y, z): d(x, y, z)=0\}$.

Substituindo nas equações de Lagrange, encontramos o ponto $\left(x_{M}, y_{M}, z_{M}\right)=$ $(0,47.6246,9.64912)$ que realiza o valor máximo para a função b nesse elipsóide. Assim, o valor máximo para b no elipsóide é $b\left(x_{M}, y_{M}, z_{M}\right)=96337.4$.

Escolhemos então $R_{1}=96400, R=96999, H=300000$ e $\delta=1$

$O$ mínimo da função $d$ é aproximadamente 594,81, $-\dot{V}(x, y, z, n, \lambda) \geq h d_{1}(x, y, z)=$ $c(x, y, z)>-596 h>596 h_{0}$. Se $h_{0}<\frac{1}{596}$ temos $c(x, y, z)>-1:=-\mu$.

Conseqüentemente, aplicando o Teorema 2.2.1 concluímos que o atrator está contido no elipsóide $A_{R+\mu}=A_{97000}=\left\{(x, y, z) \in A_{H}: r_{m} x^{2}+4 a_{m} y^{2}+4 a_{m} z^{2} \leq 97000\right\}$

Utilizando MATLAB 7.1 com a seqüência de comandos:

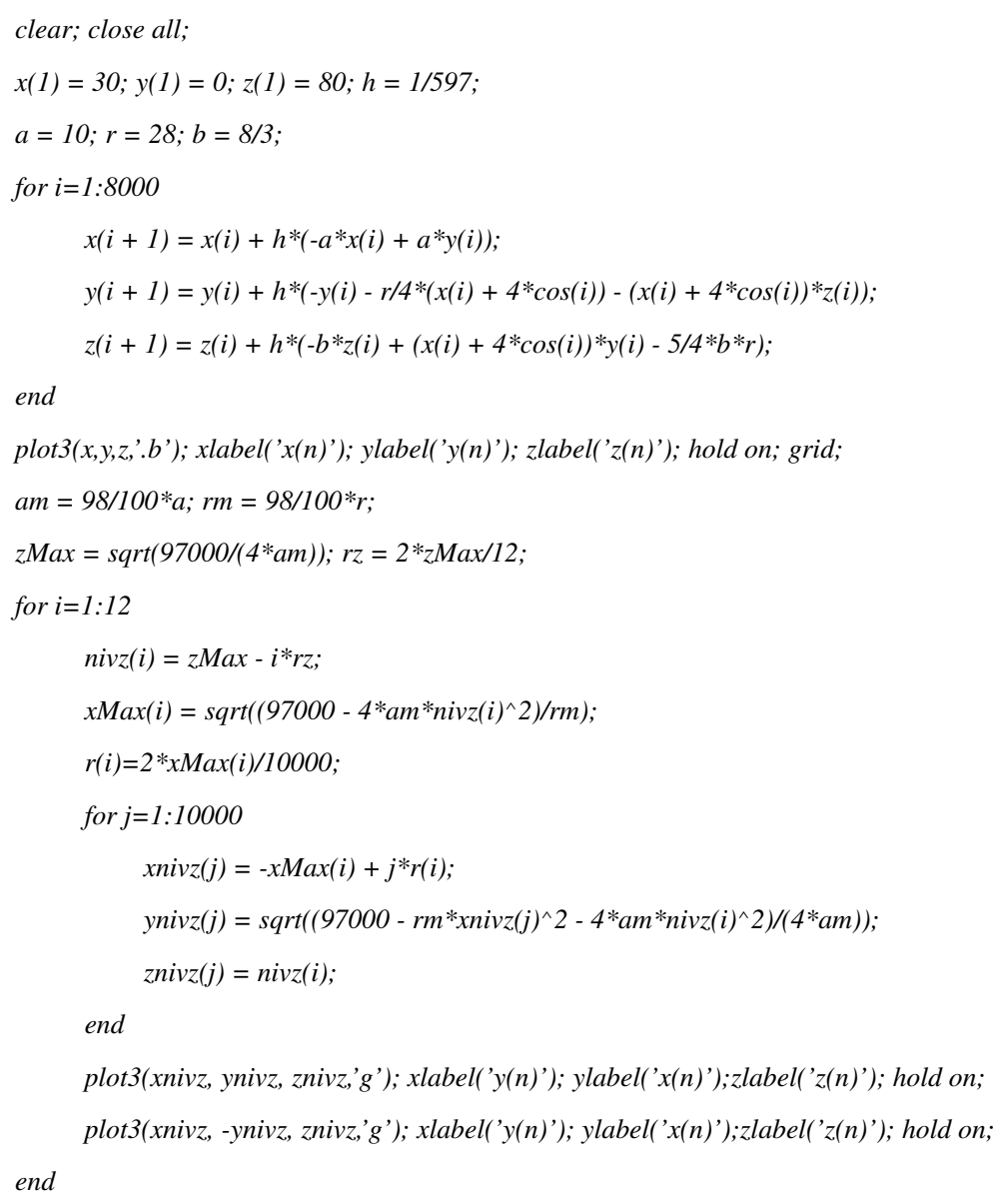


Obtivemos as seguintes imagens, que mostram o comportamento assintótico de $(x(n), y(n), z(n)), n \in \mathbb{N}$ sob diferentes ângulos:

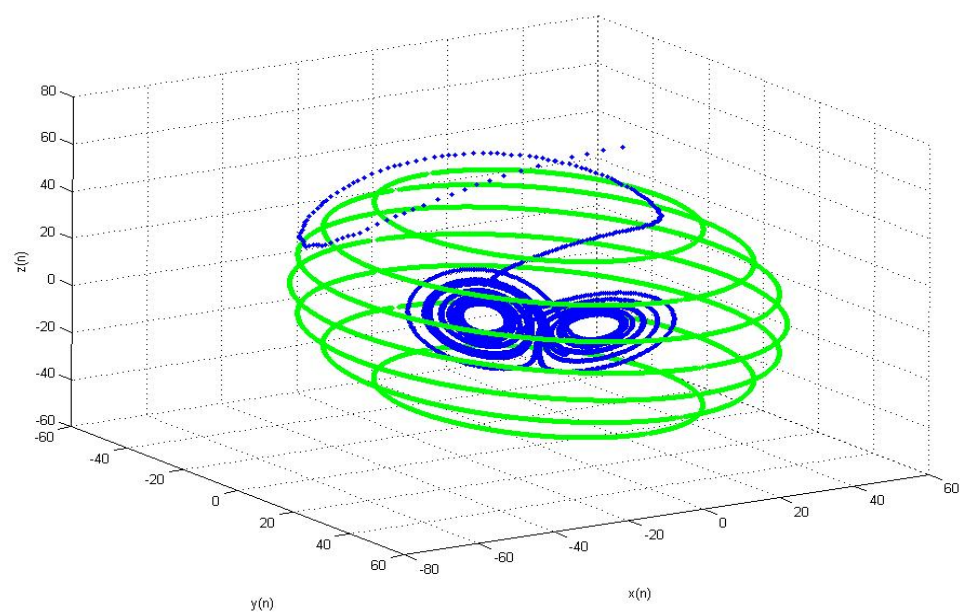

Figura 2.4: Estimativa uniforme do atrator de Lorenz.

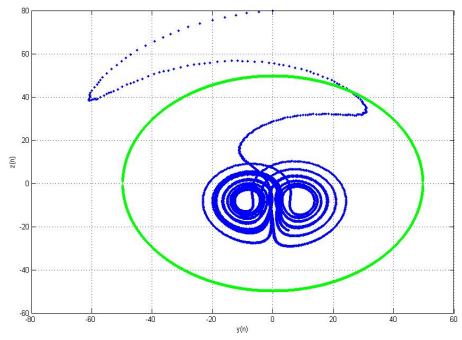

(a) Projeção em $x=0$

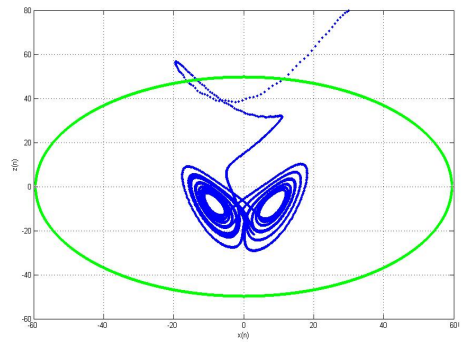

(b) Projeção em $y=0$

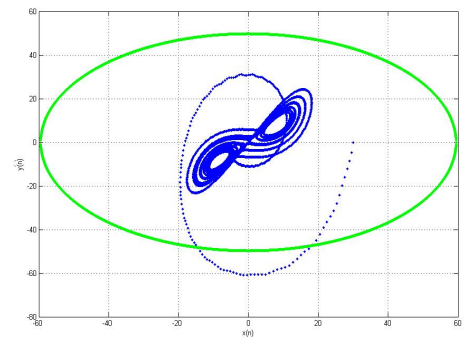

(c) Projeção em $z=0$

Figura 2.5: Projeções da estimativa uniforme do atrator de Lorenz 
Exemplo 2.3.2 (Lorenz discreto $I I$ ).

O seguinte sistema contínuo foi obtido de [32]:

$$
\left\{\begin{array}{l}
\dot{x}=-\frac{x}{4}-y^{2}-z^{2}+2 \\
\dot{y}=-y+x y-4 x z+1 \\
\dot{z}=4 x y+x z-z
\end{array}\right.
$$

Aqui consideraremos o sistema discreto correspondente via o método iterativo de Euler:

$$
\left\{\begin{array}{l}
x(n+1)=x(n)+h\left[-\frac{1}{4} x(n)-y(n)^{2}-z(n)^{2}+2\right] \\
y(n+1)=y(n)+h[-y(n)+x(n) y(n)-4 x(n) z(n)+1] \\
z(n+1)=z(n)+h[4 x(n) y(n)+x(n) z(n)-z(n)]
\end{array}\right.
$$

Apesar de não termos variação de parâmetros neste exemplo, podemos utilizar o Teorema 2.2.1 considerando que este sistema está parametrizado com um parâmetro fixo.

Para obter uma estimativa do atrator desse sistema, usaremos a função de Liapunov generalizada $V(x, y, z)=\frac{1}{2}\left[\left(x-\frac{1}{2}\right)^{2}+y^{2}+z^{2}\right]$. Utilizaremos a própria função $V$ para ser as funções a e b do Teorema 2.2.1, isto é, $a(x, y, z)=V(x, y, z)=b(x, y, z)$, para todo $(x, y, z)$ em $\mathbb{R}^{3}$, neste caso.

Como no exemplo anterior, para determinar uma função c vamos inicialmente calcular $-\dot{V}$.

$$
\begin{gathered}
-\dot{V}(x, y, z)=\frac{1}{2}\left[\left(x-\frac{1}{2}\right)^{2}+y^{2}+z^{2}\right]-\frac{1}{2}\left\{\left\{\left[x+h\left(-\frac{x}{4}-y^{2}-z^{2}+2\right)\right]-\frac{1}{2}\right\}^{2}+[y+h(-y+x y-\right. \\
\left.4 x z+1)]^{2}+[z+h(4 x y+x z-z)]^{2}\right\}= \\
\frac{1}{2}[z+h(4 x y+x z-z)]^{2}= \\
\frac{1}{2}\left(x-\frac{1}{2}\right)^{2}+\frac{1}{2} y^{2}+\frac{1}{2} z^{2}-\frac{1}{2}\left\{\left[x+h\left(-\frac{x}{4}-y^{2}-z^{2}+2\right)\right]-\frac{1}{2}\right\}^{2}-\frac{1}{2}[y+h(-y+x y-4 x z+1)]^{2}- \\
\frac{1}{2}\left(x^{2}-x+\frac{1}{4}\right)+\frac{1}{2} y^{2}+\frac{1}{2} z^{2}-\frac{1}{2}\left\{\left[x+h\left(-\frac{x}{4}-y^{2}-z^{2}+2\right)\right]^{2}-\left[x+h\left(-\frac{x}{4}-y^{2}-z^{2}+2\right)\right]+\frac{1}{4}\right\}- \\
\frac{1}{2}\left[y^{2}+2 h y(-y+x y-4 x z+1)+h^{2}(-y+x y-4 x z+1)^{2}\right]-\frac{1}{2}\left[z^{2}+2 h z(4 x y+x z-z)+h^{2}(4 x y+\right. \\
\left.x z-z)^{2}\right]= \\
\frac{1}{2} x^{2}-\frac{1}{2} x+\frac{1}{8}+\frac{1}{2} y^{2}+\frac{1}{2} z^{2}-\frac{1}{2}\left[x+h\left(-\frac{x}{4}-y^{2}-z^{2}+2\right)\right]^{2}+\frac{1}{2}\left[x+h\left(-\frac{x}{4}-y^{2}-z^{2}+2\right)\right]-\frac{1}{8}-\frac{1}{2} y^{2}- \\
h y(-y+x y-4 x z+1)-\frac{1}{2} h^{2}(-y+x y-4 x z+1)^{2}-\frac{1}{2} z^{2}-h z(4 x y+x z-z)-\frac{1}{2} h^{2}(4 x y+x z-z)^{2}= \\
\frac{1}{2} x^{2}-\frac{1}{2} x-\frac{1}{2}\left[x+h\left(-\frac{x}{4}-y^{2}-z^{2}+2\right)\right]^{2}+\frac{1}{2}\left[x+h\left(-\frac{x}{4}-y^{2}-z^{2}+2\right)\right]-h y(-y+x y-4 x z+1)- \\
\frac{1}{2} h^{2}(-y+x y-4 x z+1)^{2}-h z(4 x y+x z-z)-\frac{1}{2} h^{2}(4 x y+x z-z)^{2}= \\
\frac{1}{2} x^{2}-\frac{1}{2} x-\frac{1}{2}\left[x+h\left(-\frac{x}{4}-y^{2}-z^{2}+2\right)\right]^{2}+\frac{1}{2} x+\frac{1}{2} h\left(-\frac{x}{4}-y^{2}-z^{2}+2\right)+h y^{2}-h x y^{2}+4 h x y z- \\
h y-\frac{1}{2} h^{2}(-y+x y-4 x z+1)^{2}-4 h x y z-h x z^{2}+h z^{2}-\frac{1}{2} h^{2}(4 x y+x z-z)^{2}= \\
\frac{1}{2} x^{2}-\frac{1}{2}\left[x+h\left(-\frac{x}{4}-y^{2}-z^{2}+2\right)\right]^{2}+\frac{1}{2} h\left(-\frac{x}{4}-y^{2}-z^{2}+2\right)+h y^{2}-h x y^{2}-h y-\frac{1}{2} h^{2}(-y+x y- \\
4 x z+1)^{2}-h x z^{2}+h z^{2}-\frac{1}{2} h^{2}(4 x y+x z-z)^{2}=
\end{gathered}
$$




$$
\begin{gathered}
\frac{1}{2} x^{2}-\frac{1}{2}\left[x^{2}+2 h x\left(-\frac{x}{4}-y^{2}-z^{2}+2\right)+h^{2}\left(-\frac{x}{4}-y^{2}-z^{2}+2\right)^{2}\right]+\frac{1}{2} h\left(-\frac{x}{4}-y^{2}-z^{2}+2\right)+h y^{2}- \\
h x y^{2}-h y-\frac{1}{2} h^{2}(-y+x y-4 x z+1)^{2}-h x z^{2}+h z^{2}-\frac{1}{2} h^{2}(4 x y+x z-z)^{2}= \\
\frac{1}{2} x^{2}-\frac{1}{2} x^{2}-h x\left(-\frac{x}{4}-y^{2}-z^{2}+2\right)-\frac{1}{2} h^{2}\left(-\frac{x}{4}-y^{2}-z^{2}+2\right)^{2}-h \frac{x}{8}-\frac{1}{2} h y^{2}-\frac{1}{2} h z^{2}+h+h y^{2}- \\
h x y^{2}-h y-\frac{1}{2} h^{2}(-y+x y-4 x z+1)^{2}-h x z^{2}+h z^{2}-\frac{1}{2} h^{2}(4 x y+x z-z)^{2}= \\
-h x\left(-\frac{x}{4}-y^{2}-z^{2}+2\right)-\frac{1}{2} h^{2}\left(-\frac{x}{4}-y^{2}-z^{2}+2\right)^{2}-h \frac{x}{8}-\frac{1}{2} h y^{2}-\frac{1}{2} h z^{2}+h+h y^{2}-h x y^{2}-h y- \\
\frac{1}{2} h^{2}(-y+x y-4 x z+1)^{2}-h x z^{2}+h z^{2}-\frac{1}{2} h^{2}(4 x y+x z-z)^{2}= \\
h\left[-x\left(-\frac{x}{4}-y^{2}-z^{2}+2\right)-\frac{x}{8}-\frac{1}{2} y^{2}-\frac{1}{2} z^{2}+1+y^{2}-x y^{2}-y-x z^{2}+z^{2}\right]+h^{2}\left[-\frac{1}{2}\left(-\frac{x}{4}-y^{2}-z^{2}+\right.\right. \\
\left.2)^{2}-\frac{1}{2}(-y+x y-4 x z+1)^{2}-\frac{1}{2}(4 x y+x z-z)^{2}\right]=
\end{gathered}
$$

Assim:

$$
\begin{gathered}
-\frac{\dot{V}}{h}=-x\left(-\frac{x}{4}-y^{2}-z^{2}+2\right)-\frac{x}{8}-\frac{1}{2} y^{2}-\frac{1}{2} z^{2}+1+y^{2}-x y^{2}-y-x z^{2}+z^{2}+h\left[-\frac{1}{2}\left(-\frac{x}{4}-y^{2}-\right.\right. \\
\left.\left.z^{2}+2\right)^{2}-\frac{1}{2}(-y+x y-4 x z+1)^{2}-\frac{1}{2}(4 x y+x z-z)^{2}\right]= \\
\frac{x^{2}}{4}+x y^{2}+x z^{2}-2 x-\frac{x}{8}-\frac{1}{2} y^{2}-\frac{1}{2} z^{2}+1+y^{2}-x y^{2}-y-x z^{2}+z^{2}+h g(x, y, z)= \\
\frac{x^{2}}{4}+\frac{1}{2} y^{2}+\frac{1}{2} z^{2}-\frac{17}{8} x-y+1+h g(x, y, z) \geq \\
\frac{x^{2}}{4}+\frac{1}{2} y^{2}+\frac{1}{2} z^{2}-\frac{17}{8}|x|-|y|+1-|h g(x, y, z)|
\end{gathered}
$$

Seja $d(x, y, z)=\frac{x^{2}}{4}+\frac{1}{2} y^{2}+\frac{1}{2} z^{2}-\frac{17}{8}|x|-|y|+1$. Completando quadrados, temos:

$d(x, y, z)=\frac{1}{4}\left(x^{2}-\frac{17}{2}|x|+\frac{289}{16}\right)-\frac{289}{64}+\frac{1}{2}\left(y^{2}-2|y|+1\right)-\frac{1}{2}+\frac{1}{2} z^{2}+1=\frac{1}{4}\left(|x|-\frac{17}{4}\right)^{2}+$
$\frac{1}{2}(|y|-1)^{2}+\frac{1}{2} z^{2}-\frac{257}{64}$

Seja $\mathcal{D}:=\{(x, y, z): d(x, y, z) \leq 0\}$ e sejam $R_{1}$ e $R$ tais que $\max \{b(x, y, z) ;(x, y, z) \in \mathcal{D}\}<$ $R_{1}<R$. Escolheremos $H$ tão grande quanto $R$.

É importante determinar o máximo de $b(x, y, z)$ em $\mathcal{D}$ que irá ocorrer na fronteira de $\mathcal{D}$, que é um elipsóide. Pelo método dos multiplicadores de Lagrange:

$$
\left\{\begin{array}{cc}
V_{x} & =\lambda b_{x} \\
V_{y} & =\lambda b_{y} \\
V_{z} & =\lambda b_{z} \\
d(x, y, z) & =0
\end{array}\right.
$$

obtemos o sistema:

$$
\left\{\begin{array}{ccc}
x-\frac{1}{2} & = & \lambda \frac{1}{2}\left(x-\frac{17}{4}\right) \\
y & = & \lambda(y-1) \\
z & = & \lambda z \\
\frac{1}{4}\left(x-\frac{17}{4}\right)^{2}+\frac{1}{2}(y-1)^{2}+\frac{1}{2} z^{2} & = & \frac{257}{64}
\end{array}\right.
$$


De (3), $\lambda=1 \forall z$ (não por (2)) ou $z=0 \forall \lambda$; de (2), $y=\frac{1}{\lambda-1}$; de (1), $x=\frac{17 \lambda-4}{4 \lambda-8}$; substituindo em (4):

$$
\begin{gathered}
\frac{1}{4}\left(\frac{17 \lambda-4}{4 \lambda-8}-\frac{17}{4}\right)^{2}+\frac{1}{2}\left(\frac{1}{\lambda-1}-1\right)^{2}=\frac{257}{64} \Rightarrow \frac{1}{4}\left(\frac{-15}{4 \lambda-2}\right)^{2}+\frac{1}{2}\left(\frac{-\lambda+2}{\lambda-1}\right)^{2}=\frac{257}{64} \Rightarrow \\
\frac{225}{16 \lambda^{2}-64 \lambda+64}+\frac{\lambda^{2}-4 \lambda+4}{2 \lambda^{2}-4 \lambda+2}=\frac{257}{64} \Rightarrow \frac{\left(450 \lambda^{2}-900 \lambda+450\right)+\left(16 \lambda^{4}-128 \lambda^{3}+384 \lambda^{2}-512 \lambda+256\right)}{32 \lambda^{4}-192 \lambda^{3}+416 \lambda^{2}-384 \lambda+128}=\frac{257}{64} \\
\frac{16 \lambda^{4}-128 \lambda^{3}+834 \lambda^{2}-1412 \lambda+706}{32 \lambda^{4}-192 \lambda^{3}+416 \lambda^{2}-384 \lambda+128}=\frac{257}{64} \Rightarrow \\
257\left(\lambda^{4}-6 \lambda^{3}+13 \lambda^{2}-12 \lambda+4\right)=2\left(16 \lambda^{4}-128 \lambda^{3}+834 \lambda^{2}-1412 \lambda+706\right) \Rightarrow \\
257 \lambda^{4}-1542 \lambda^{3}+3341 \lambda^{2}-3084 \lambda-1028=32 \lambda^{4}-256 \lambda^{3}+1668 \lambda^{2}-2824 \lambda+1412 \Rightarrow \\
225 \lambda^{4}-1286 \lambda^{3}+1673 \lambda^{2}-260 \lambda-2440=0 \Rightarrow
\end{gathered}
$$

Este polinômio foi resolvido numericamente usando MATHEMATICA, e foram encontradas as seguintes raízes $4.139685744,-0.8657600199$ e mais duas raízes complexas. A primeira dá o máximo para $V$ em $\Gamma^{-1}(0)$, e a segunda dá o mínimo.

Substituindo nas equações de Lagrange, encontramos $x=7.755187626, y=0.3185032139$ $e z=0$. Após alguns cálculos, obtemos que o máximo que $V$ assume é 26.36959590.

Assim, o atrator está contido no elipsóide $A_{27}=\left\{(x, y, z) \in \mathbb{R}^{3}: \frac{1}{2}\left(x-\frac{1}{2}\right)^{2}+\frac{1}{2} y^{2}+\frac{1}{2} z^{2} \leq\right.$ $27\}$.

Utilizando MATLAB 7.1 com a seqüência de comandos:

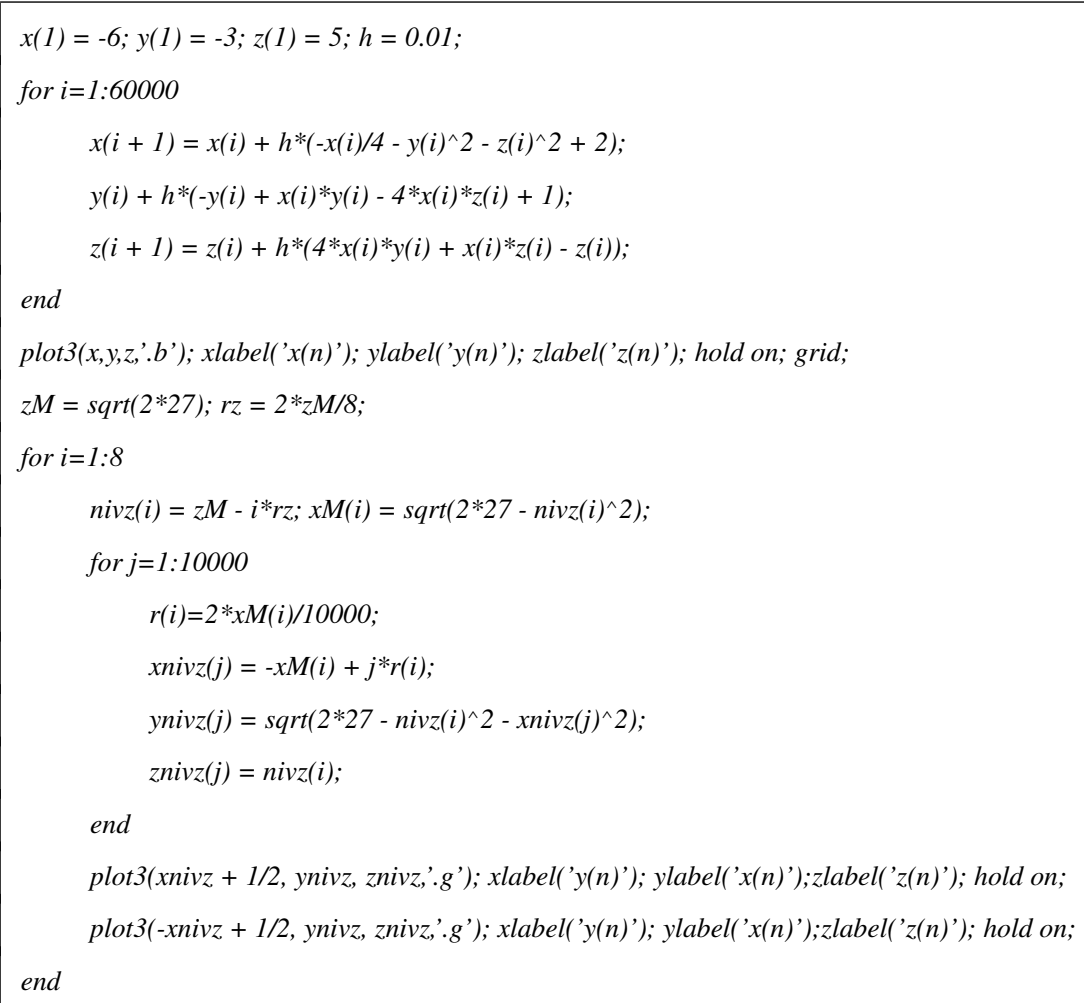




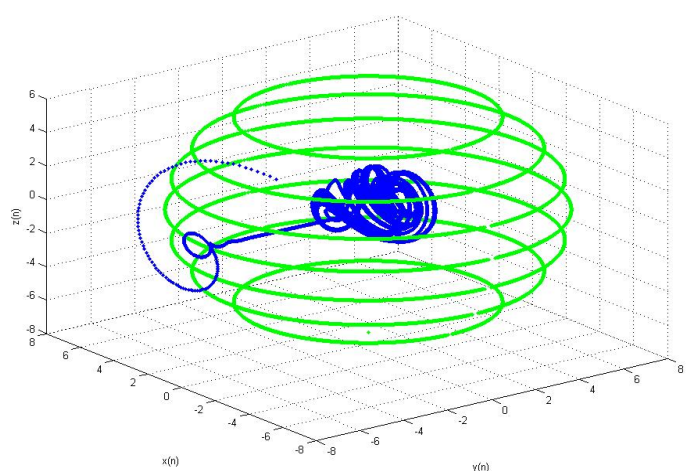

(a) Estimativa do atrator

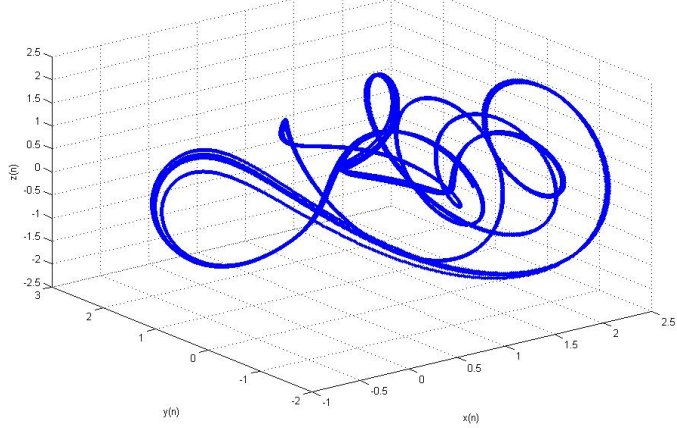

(b) Atrator: nesta figura plotei os ultimos 20000 pontos

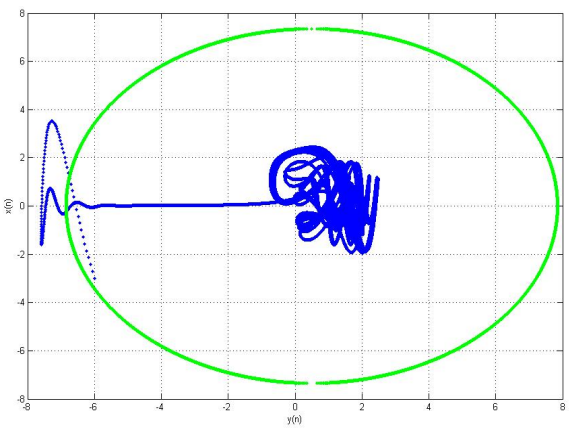

(c) Projeção em y = 0

Figura 2.6:

\section{Exemplo 2.3.3.}

O sistema contínuo $x^{\prime \prime}+\alpha x^{\prime}+\omega x+\left(q \cos (t)+x^{2}\right) x=0$ foi estudado por Afraimovich (Veja [1]), e é equivalente a:

$$
\left\{\begin{array}{l}
x^{\prime}=y \\
y^{\prime}=-\alpha y-\omega x-\left(q \cos (t)+x^{2}\right) x
\end{array} .\right.
$$

Considere o sistema discreto correspondente (via método iterativo de Euler).

$$
\left\{\begin{array}{l}
x(n+1)=x(n)+h y(n) \\
y(n+1)=y(n)+h\left\{-\alpha y(n)-\omega x(n)-\left(q \cos (n)+x(n)^{2}\right) x(n)\right\}
\end{array}\right.
$$

Onde $\lambda:=(\alpha, \omega, q, t)$.

Para determinar uma estimativa do atrator de 2.3, usaremos a função de Liapunov $V(x, y)=\frac{1}{2} \omega x^{2}+\frac{1}{4} x^{4}+\frac{1}{2} y^{2}+\varepsilon x y$.

É fácil ver que $a(x, y) \leq V(x, y) \leq b(x, y)$, onde $a(x, y)=\frac{1}{2} \omega_{m} x^{2}+\frac{1}{4} x^{4}+\frac{1}{2} y^{2}-\varepsilon|x y| e$ $b(x, y)=\frac{1}{2} \omega_{M} x^{2}+\frac{1}{4} x^{4}+\frac{1}{2} y^{2}+\varepsilon|x y|$, então: 


$$
\begin{aligned}
& -\dot{V}(x, y)=\left(\frac{1}{2} \omega x^{2}+\frac{1}{4} x^{4}+\frac{1}{2} y^{2}+\varepsilon x y\right)-\left\{\frac{1}{2} \omega(x+h y)^{2}+\frac{1}{4}(x+h y)^{4}+\frac{1}{2}\{y+h[-\alpha y-\omega x-\right. \\
& \left.\left.\left.\left(q \cos (t)+x^{2}\right) x\right]\right\}^{2}+\varepsilon(x+h y)\left\{y+h\left[-\alpha y-\omega x-\left(q \cos (t)+x^{2}\right) x\right]\right\}\right\}= \\
& \frac{1}{2} \omega x^{2}+\frac{1}{4} x^{4}+\frac{1}{2} y^{2}+\varepsilon x y-\frac{1}{2} \omega(x+h y)^{2}-\frac{1}{4}(x+h y)^{4}-\frac{1}{2}\{y+h[-\alpha y-\omega x-(q \cos (t)+ \\
& \left.\left.\left.x^{2}\right) x\right]\right\}^{2}-\varepsilon(x+h y)\left\{y+h\left[-\alpha y-\omega x-\left(q \cos (t)+x^{2}\right) x\right]\right\}= \\
& \frac{1}{2} \omega x^{2}+\frac{1}{4} x^{4}+\frac{1}{2} y^{2}+\varepsilon x y-\frac{1}{2} \omega\left(x^{2}+2 h x y+h^{2} y^{2}\right)-\frac{1}{4}\left(x^{4}+4 h x^{3} y+6 h^{2} x^{2} y^{2}+4 h^{3} x y^{3}+h^{4} y^{4}\right)- \\
& \frac{1}{2}\left\{y^{2}+2 h y\left[-\alpha y-\omega x-\left(q \cos (t)+x^{2}\right) x\right]+h^{2}\left[-\alpha y-\omega x-\left(q \cos (t)+x^{2}\right) x\right]^{2}\right\}-(\varepsilon x+ \\
& \varepsilon h y)\left\{y+h\left[-\alpha y-\omega x-\left(q \cos (t)+x^{2}\right) x\right]\right\}= \\
& \frac{1}{2} \omega x^{2}+\frac{1}{4} x^{4}+\frac{1}{2} y^{2}+\varepsilon x y-\frac{1}{2} \omega x^{2}-\omega h x y-\frac{1}{2} \omega h^{2} y^{2}-\frac{1}{4} x^{4}-h x^{3} y-\frac{3}{2} h^{2} x^{2} y^{2}-h^{3} x y^{3}-\frac{1}{4} h^{4} y^{4}- \\
& \frac{1}{2} y^{2}-h y\left[-\alpha y-\omega x-\left(q \cos (t)+x^{2}\right) x\right]-\frac{1}{2} h^{2}\left[-\alpha y-\omega x-\left(q \cos (t)+x^{2}\right) x\right]^{2}-\{\varepsilon x y+ \\
& \left.\varepsilon h x\left[-\alpha y-\omega x-\left(q \cos (t)+x^{2}\right) x\right]+\varepsilon h y^{2}+\varepsilon h^{2} y\left[-\alpha y-\omega x-\left(q \cos (t)+x^{2}\right) x\right]\right\}= \\
& \varepsilon x y-\omega h x y-\frac{1}{2} \omega h^{2} y^{2}-h x^{3} y-\frac{3}{2} h^{2} x^{2} y^{2}-h^{3} x y^{3}-\frac{1}{4} h^{4} y^{4}-h y\left[-\alpha y-\omega x-\left(q \cos (t)+x^{2}\right) x\right]- \\
& \frac{1}{2} h^{2}\left[-\alpha y-\omega x-\left(q \cos (t)+x^{2}\right) x\right]^{2}-\left\{\varepsilon x y+\varepsilon h x\left[-\alpha y-\omega x-\left(q \cos (t)+x^{2}\right) x\right]+\varepsilon h y^{2}+\right. \\
& \left.\varepsilon h^{2} y\left[-\alpha y-\omega x-\left(q \cos (t)+x^{2}\right) x\right]\right\}= \\
& \varepsilon x y-\omega h x y-h x^{3} y+\alpha h y^{2}+\omega h x y+h\left(q \cos (t)+x^{2}\right) x y-\varepsilon x y-\varepsilon h x[-\alpha y-\omega x-(q \cos (t)+ \\
& \left.\left.x^{2}\right) x\right]-\varepsilon h y^{2}+h^{2}\left\{-\frac{1}{2} \omega y^{2}-\frac{3}{2} x^{2} y^{2}-h x y^{3}-\frac{1}{4} h^{2} y^{4}-\frac{1}{2}\left[-\alpha y-\omega x-\left(q \cos (t)+x^{2}\right) x\right]^{2}-\right. \\
& \left.\varepsilon y\left[-\alpha y-\omega x-\left(q \cos (t)+x^{2}\right) x\right]\right\}= \\
& h\left\{-x^{3} y+\alpha y^{2}+\left(q \cos (t)+x^{2}\right) x y-\varepsilon x\left[-\alpha y-\omega x-\left(q \cos (t)+x^{2}\right) x\right]-\varepsilon y^{2}\right\}+h^{2}\left\{-\frac{1}{2} \omega y^{2}-\right. \\
& \left.\frac{3}{2} x^{2} y^{2}-h x y^{3}-\frac{1}{4} h^{2} y^{4}-\frac{1}{2}\left[-\alpha y-\omega x-\left(q \cos (t)+x^{2}\right) x\right]^{2}-\varepsilon y\left[-\alpha y-\omega x-\left(q \cos (t)+x^{2}\right) x\right]\right\}= \\
& h\left\{-x^{3} y+\alpha y^{2}+\left(q \cos (t)+x^{2}\right) x y+\alpha \varepsilon x y+\varepsilon \omega x^{2}+\varepsilon\left(q \cos (t)+x^{2}\right) x^{2}-\varepsilon y^{2}\right\}+o\left(h^{2}\right) \\
& -x^{3} y+\alpha y^{2}+q \cos (t) x y+x^{3} y+\alpha \varepsilon x y+\varepsilon \omega x^{2}+\varepsilon q \cos (t) x^{2}+\varepsilon x^{4}-\varepsilon y^{2}+o(h)= \\
& \alpha y^{2}+q \cos (t) x y+\alpha \varepsilon x y+\varepsilon \omega x^{2}+\varepsilon q \cos (t) x^{2}+\varepsilon x^{4}-\varepsilon y^{2}+o(h) \geq \\
& \alpha y^{2}-q|x y|-\alpha \varepsilon|x y|+\varepsilon \omega x^{2}-\varepsilon q x^{2}+\varepsilon x^{4}-\varepsilon y^{2}+o(h) \geq \\
& \alpha y^{2}-q_{M}|x||y|-\alpha_{M} \varepsilon|x||y|+\varepsilon \omega_{m} x^{2}-\varepsilon q_{M} x^{2}+\varepsilon x^{4}-\varepsilon y^{2}+o(h)= \\
& \varepsilon x^{4}-\underbrace{\varepsilon\left(q_{M}-\omega_{m}\right)}_{A} x^{2}-\underbrace{\left(q_{M}+\alpha_{M} \varepsilon\right)}_{B}|x||y|+(\alpha-\varepsilon) y^{2}+o(h) \geq \\
& \varepsilon x^{4}-A x^{2}-B|x||y|+\varepsilon y^{2}+o(h)
\end{aligned}
$$

Seja $d(x, y):=\varepsilon x^{4}-A x^{2}-B|x||y|+\varepsilon y^{2} ;$ completando quadrados, temos:

$$
\varepsilon x^{4}-A x^{2}+\varepsilon\left(y^{2}-B|x||y|\right)=
$$




$$
\begin{gathered}
\varepsilon x^{4}-A x^{2}+\varepsilon\left[y^{2}-2 \frac{B}{2 \varepsilon}|x||y|+\left(\frac{B}{2 \varepsilon}\right)^{2} x^{2}\right]-\frac{B^{2}}{4 \varepsilon} x^{2}= \\
x^{2}[\varepsilon x^{2}-\underbrace{\left(A+\frac{B^{2}}{4 \varepsilon}\right)}_{C}]+\varepsilon\left[|y|-\frac{B}{2 \varepsilon}|x|\right]^{2}
\end{gathered}
$$

Como $\varepsilon\left[|y|-\frac{B}{2 \varepsilon}|x|\right]^{2} \geq 0$ para todo $x$ e para todo $y$, o mínimo de dé atingido quando este termo se anula e $x^{2}\left[\varepsilon x^{2}-C\right]$ atinge seu mínimo.

Pelo método da primeira e segunda derivadas, encontramos os pontos críticos de $x^{2}\left[\varepsilon x^{2}-\right.$ $C]$.

0 é o ponto de máximo e o mínimo ocorre em $\pm \sqrt{\frac{c}{2 \varepsilon}}$.

O valor mínimo de $x^{2}\left[\varepsilon x^{2}-C\right] e ́$ :

$\left(\sqrt{\frac{c}{2 \varepsilon}}\right)^{2}\left[\varepsilon\left(\sqrt{\frac{c}{2 \varepsilon}}\right)^{2}-C\right]=\frac{c}{2 \varepsilon}\left[\varepsilon \frac{c}{2 \varepsilon}-C\right]=\frac{c}{2 \varepsilon}\left[\frac{c}{2}-C\right]=\frac{c}{2 \varepsilon}\left[-\frac{c}{2}\right]=-\frac{c^{2}}{4 \varepsilon}$

Conseqüentemente, $\frac{-\dot{V}}{h}(x, y) \geq-\frac{c^{2}}{4 \varepsilon}+\varepsilon\left[|y|-\frac{B}{2 \varepsilon}|x|\right]^{2}$.

$-\frac{c^{2}}{4 \varepsilon}+\varepsilon\left[|y|-\frac{B}{2 \varepsilon}|x|\right]^{2}=0 \Rightarrow$

$\varepsilon\left[|y|-\frac{B}{2 \varepsilon}|x|\right]^{2}=\frac{c^{2}}{4 \varepsilon} \Rightarrow|y|-\frac{B}{2 \varepsilon}|x|= \pm \frac{C}{2 \varepsilon}$.

Segue que a região onde $d(x, y)<0$ é limitada.

Pela simetria da função||, é suficiente descrevermos o conjunto dos pontos do primeiro quadrante que cumprem essa desigualdade, que os demais serão obtidos por reflexão destes com respeito aos eixos coordenados. Assim é suficiente plotarmos o seguinte par de retas no primeiro quadrante: $y-\frac{B}{2 \varepsilon} x= \pm \frac{C}{2 \varepsilon}$.

I) $y-\frac{B}{2 \varepsilon} x=-\frac{C}{2 \varepsilon}$ :

$$
\begin{aligned}
& \text { Se } y=0 \text { então }-\frac{B}{2 \varepsilon} x=-\frac{C}{2 \varepsilon} \Rightarrow B x=C \Rightarrow x=\frac{C}{B} . \\
& \text { Se } x=\sqrt{\frac{c}{\varepsilon}} \text { então } y-\frac{B}{2 \varepsilon} \sqrt{\frac{c}{\varepsilon}}=-\frac{C}{2 \varepsilon} \Rightarrow y=\frac{B}{2 \varepsilon} \sqrt{\frac{c}{\varepsilon}}-\frac{C}{2 \varepsilon}=\frac{1}{2 \varepsilon}\left(B \sqrt{\frac{c}{\varepsilon}}-C\right) .
\end{aligned}
$$

II) $y-\frac{B}{2 \varepsilon} x=\frac{C}{2 \varepsilon}$ :

$$
\begin{aligned}
& \text { Se } x=0 \text { então } y=\frac{C}{2 \varepsilon} . \\
& \text { Se } x=\sqrt{\frac{c}{\varepsilon}} \text { então } y-\frac{B}{2 \varepsilon} \sqrt{\frac{c}{\varepsilon}}=\frac{C}{2 \varepsilon} \Rightarrow y=\frac{B}{2 \varepsilon} \sqrt{\frac{c}{\varepsilon}}+\frac{C}{2 \varepsilon}=\frac{1}{2 \varepsilon}\left(B \sqrt{\frac{c}{\varepsilon}}+C\right) .
\end{aligned}
$$

É importante encontrarmos o máximo de $b(x, y)$ em $\mathcal{D}=\left\{(x, y) \in \mathbb{R}^{2}: d(x, y) \leq 0\right\}$ o qual vai ocorrer na fronteira de $\mathcal{D}$, visto que $b$ é uma função convexa.

Se $b(x, y)=\frac{1}{2} \omega_{M} x^{2}+\frac{1}{4} x^{4}+\frac{1}{2} y^{2}+\varepsilon x y$ e $\varphi(x, y)=\sqrt{-x^{4}+\frac{C}{\varepsilon} x^{2}}+\frac{B}{2 \varepsilon} x-y$, pelo método dos multiplicadores de Lagrange: 


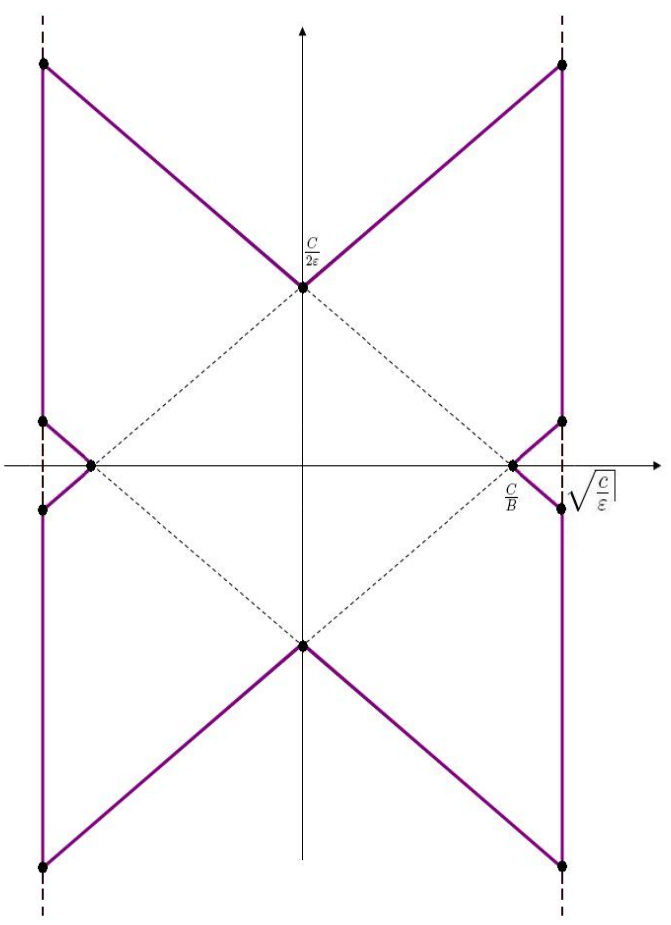

Figura 2.7: Como pode ser observado, a região onde $d(x, y)<0$ é limitada.

$$
\left\{\begin{array}{cc}
b_{x} & =\lambda \varphi_{x} \\
b_{y} & =\lambda \varphi_{y} \\
\varphi(x, y) & =0
\end{array}\right.
$$

obtemos o sistema:

$$
\left\{\begin{array}{ccc}
\omega_{M} x+x^{3}+\varepsilon y & = & \lambda\left[\frac{1}{2}\left(-4 x^{3}+\frac{2 C}{\varepsilon} x\right)\left(-x^{4}+\frac{C}{\varepsilon} x^{2}\right)^{-\frac{1}{2}}+\frac{B}{2 \varepsilon}\right] \\
y+\varepsilon x & = & -\lambda \\
y & = & \sqrt{-x^{4}+\frac{C}{\varepsilon} x^{2}}+\frac{B}{2 \varepsilon} x
\end{array}\right.
$$

De (2), $\lambda=-y-\varepsilon x$. Substituindo em (1):

$$
\begin{gathered}
\omega_{M} x+x^{3}+\varepsilon y=(-y-\varepsilon x)\left[\frac{1}{2}\left(-4 x^{3}+\frac{2 C}{\varepsilon} x\right)\left(-x^{4}+\frac{C}{\varepsilon} x^{2}\right)^{-\frac{1}{2}}+\frac{B}{2 \varepsilon}\right] \Rightarrow \\
\omega_{M} x+x^{3}+\varepsilon y= \\
-y\left[\frac{1}{2}\left(-4 x^{3}+\frac{2 C}{\varepsilon} x\right)\left(-x^{4}+\frac{C}{\varepsilon} x^{2}\right)^{-\frac{1}{2}}\right]-\varepsilon x\left[\frac{1}{2}\left(-4 x^{3}+\frac{2 C}{\varepsilon} x\right)\left(-x^{4}+\frac{C}{\varepsilon} x^{2}\right)^{-\frac{1}{2}}\right]-\frac{B}{2 \varepsilon} y-\frac{B}{2} x \Rightarrow \\
\omega_{M} x+x^{3}+\varepsilon y= \\
\frac{-y}{2}\left[\left(-4 x^{3}+\frac{2 C}{\varepsilon} x\right)\left(-x^{4}+\frac{C}{\varepsilon} x^{2}\right)^{-\frac{1}{2}}\right]-\frac{\varepsilon x}{2}\left[\left(-4 x^{3}+\frac{2 C}{\varepsilon} x\right)\left(-x^{4}+\frac{C}{\varepsilon} x^{2}\right)^{-\frac{1}{2}}\right]-\frac{B}{2 \varepsilon} y-\frac{B}{2} x \Rightarrow \\
\omega_{M} x+x^{3}+\varepsilon y=\frac{-y}{2}\left[-4 x^{3}\left(-x^{4}+\frac{C}{\varepsilon} x^{2}\right)^{-\frac{1}{2}}+\frac{2 C}{\varepsilon} x\left(-x^{4}+\frac{C}{\varepsilon} x^{2}\right)^{-\frac{1}{2}}\right]-\frac{\varepsilon}{2} x\left[-4 x^{3}\left(-x^{4}+\frac{C}{\varepsilon} x^{2}\right)^{-\frac{1}{2}}+\right. \\
\left.\frac{2 C}{\varepsilon} x\left(-x^{4}+\frac{C}{\varepsilon} x^{2}\right)^{-\frac{1}{2}}\right]-\frac{B}{2 \varepsilon} y-\frac{B}{2} x \Rightarrow
\end{gathered}
$$




$$
\begin{gathered}
\omega_{M} x+x^{3}+\varepsilon y=2 x^{3} y\left(-x^{4}+\frac{C}{\varepsilon} x^{2}\right)^{-\frac{1}{2}}-\frac{C}{\varepsilon} x y\left(-x^{4}+\frac{C}{\varepsilon} x^{2}\right)^{-\frac{1}{2}}+2 \varepsilon x^{4}\left(-x^{4}+\frac{C}{\varepsilon} x^{2}\right)^{-\frac{1}{2}}-C x^{2}\left(-x^{4}+\right. \\
\left.\frac{C}{\varepsilon} x^{2}\right)^{-\frac{1}{2}}-\frac{B}{2 \varepsilon} y-\frac{B}{2} x \Rightarrow
\end{gathered}
$$

$D e(3), y=\sqrt{-x^{4}+\frac{C}{\varepsilon} x^{2}}+\frac{B}{2 \varepsilon} x$

$$
\begin{gathered}
\omega_{M} x+x^{3}+\varepsilon\left[\left(-x^{4}+\frac{C}{\varepsilon} x^{2}\right)^{\frac{1}{2}}+\frac{B}{2 \varepsilon} x\right]= \\
2 x^{3}\left[\left(-x^{4}+\frac{C}{\varepsilon} x^{2}\right)^{\frac{1}{2}}+\frac{B}{2 \varepsilon} x\right]\left(-x^{4}+\frac{C}{\varepsilon} x^{2}\right)^{-\frac{1}{2}}-\frac{C}{\varepsilon} x\left[\left(-x^{4}+\frac{C}{\varepsilon} x^{2}\right)^{\frac{1}{2}}+\frac{B}{2 \varepsilon} x\right]\left(-x^{4}+\frac{C}{\varepsilon} x^{2}\right)^{-\frac{1}{2}}+ \\
2 \varepsilon x^{4}\left(-x^{4}+\frac{C}{\varepsilon} x^{2}\right)^{-\frac{1}{2}}-C x^{2}\left(-x^{4}+\frac{C}{\varepsilon} x^{2}\right)^{-\frac{1}{2}}-\frac{B}{2 \varepsilon}\left[\left(-x^{4}+\frac{C}{\varepsilon} x^{2}\right)^{\frac{1}{2}}+\frac{B}{2 \varepsilon} x\right]-\frac{B}{2} x \Rightarrow \\
\omega_{M} x+x^{3}+\varepsilon\left(-x^{4}+\frac{C}{\varepsilon} x^{2}\right)^{\frac{1}{2}}+\frac{B}{2} x= \\
{\left[2 x^{3}\left(-x^{4}+\frac{C}{\varepsilon} x^{2}\right)^{\frac{1}{2}}+\frac{B}{\varepsilon} x^{4}\right]\left(-x^{4}+\frac{C}{\varepsilon} x^{2}\right)^{-\frac{1}{2}}+\left[-\frac{C}{\varepsilon} x\left(-x^{4}+\frac{C}{\varepsilon} x^{2}\right)^{\frac{1}{2}}-\frac{B C}{2 \varepsilon^{2}} x^{2}\right]\left(-x^{4}+\frac{C}{\varepsilon} x^{2}\right)^{-\frac{1}{2}}+} \\
2 \varepsilon x^{4}\left(-x^{4}+\frac{C}{\varepsilon} x^{2}\right)^{-\frac{1}{2}}-C x^{2}\left(-x^{4}+\frac{C}{\varepsilon} x^{2}\right)^{-\frac{1}{2}}-\frac{B}{2 \varepsilon}\left(-x^{4}+\frac{C}{\varepsilon} x^{2}\right)^{\frac{1}{2}}-\frac{B^{2}}{4 \varepsilon^{2}} x-\frac{B}{2} x \Rightarrow \\
\omega_{M} x+x^{3}+\varepsilon\left(-x^{4}+\frac{C}{\varepsilon} x^{2}\right)^{\frac{1}{2}}+\frac{B}{2} x=2 x^{3}+\frac{B}{\varepsilon} x^{4}\left(-x^{4}+\frac{C}{\varepsilon} x^{2}\right)^{-\frac{1}{2}}-\frac{C}{\varepsilon} x-\frac{B C}{2 \varepsilon^{2}} x^{2}\left(-x^{4}+\frac{C}{\varepsilon} x^{2}\right)^{-\frac{1}{2}}+ \\
2 \varepsilon x^{4}\left(-x^{4}+\frac{C}{\varepsilon} x^{2}\right)^{-\frac{1}{2}}-C x^{2}\left(-x^{4}+\frac{C}{\varepsilon} x^{2}\right)^{-\frac{1}{2}}-\frac{B}{2 \varepsilon}\left(-x^{4}+\frac{C}{\varepsilon} x^{2}\right)^{\frac{1}{2}}-\frac{B^{2}}{4 \varepsilon^{2}} x-\frac{B}{2} x \Rightarrow \\
-x^{3}+\left(\omega_{M}+B+\frac{B^{2}}{4 \varepsilon^{2}}+\frac{C}{\varepsilon}\right) x= \\
\left(\frac{B}{\varepsilon} x^{4}+2 \varepsilon x^{4}-\frac{B C}{2 \varepsilon^{2}} x^{2}-C x^{2}\right)\left(-x^{4}+\frac{C}{\varepsilon} x^{2}\right)^{-\frac{1}{2}}-\left(\varepsilon+\frac{B}{2 \varepsilon}\right)\left(-x^{4}+\frac{C}{\varepsilon} x^{2}\right)^{\frac{1}{2}} \Rightarrow \\
x\left[-x^{2}+\left(\omega_{M}+B+\frac{B^{2}}{4 \varepsilon^{2}}+\frac{C}{\varepsilon}\right)\right]\left(-x^{4}+\frac{C}{\varepsilon} x^{2}\right)^{\frac{1}{2}}= \\
\left(\frac{B}{\varepsilon} x^{4}+2 \varepsilon x^{4}-\frac{B C}{2 \varepsilon^{2}} x^{2}-C x^{2}\right)-\left(\varepsilon+\frac{B}{2 \varepsilon}\right)\left(-x^{4}+\frac{C}{\varepsilon} x^{2}\right) \Rightarrow \\
S e x \neq 0 \\
{\left[-x^{2}+\left(\omega_{M}+B+\frac{B^{2}}{4 \varepsilon^{2}}+\frac{C}{\varepsilon}\right)\right]\left(-x^{2}+\frac{C}{\varepsilon}\right)^{\frac{1}{2}}=\left(\frac{B}{\varepsilon} x^{2}+2 \varepsilon x^{2}-\frac{B C}{2 \varepsilon^{2}}-C\right)-\left(\varepsilon+\frac{B}{2 \varepsilon}\right)\left(-x^{2}+\frac{C}{\varepsilon}\right) \Rightarrow}
\end{gathered}
$$

Seja $g(x, y):=\left[-x^{2}+\left(\omega_{M}+B+\frac{B^{2}}{4 \varepsilon^{2}}+\frac{C}{\varepsilon}\right)\right]\left(-x^{2}+\frac{C}{\varepsilon}\right)^{\frac{1}{2}}-\left(\frac{B}{\varepsilon} x^{2}+2 \varepsilon x^{2}-\frac{B C}{2 \varepsilon^{2}}-C\right)+(\varepsilon+$ $\left.\frac{B}{2 \varepsilon}\right)\left(-x^{2}+\frac{C}{\varepsilon}\right)$.

$g(x, y)=0$ foi resolvida numericamente usando MATHEMATICA, e considerando que os parâmetros variam com uma incerteza de $2 \%$ com relação aos valores básicos $\alpha=1, \omega=1$, $q=50$,

$$
\begin{gathered}
\alpha_{m}=\left(1-\frac{2}{100}\right) \alpha=\frac{49}{50}, \quad \alpha_{M}=\left(1+\frac{2}{100}\right) \alpha=\frac{51}{50}, \\
\omega_{m}=\left(1-\frac{2}{100}\right) \omega=\frac{49}{50}, \quad \omega_{M}=\left(1+\frac{2}{100}\right) \omega=\frac{51}{50}, \\
q_{m}=50-1=49, \quad q_{M}=50+1=51, \\
\varepsilon=\frac{\alpha_{M}}{2} .
\end{gathered}
$$

Foram encontradas as seguintes raízes: - $14.829399057215626 \cdots, 14.829399057215626 \cdots$.

Assim, o valor máximo que b atinge é $71141,76452862348 \cdots$.

Tomando $R_{1}=71141,76452862348$ e $R=71142$, concluimos que para $h$ suficientemente pequeno, o atrator está contido no conjunto $A_{R+1}=\{(x, y): a(x, y)<71143\}$. 


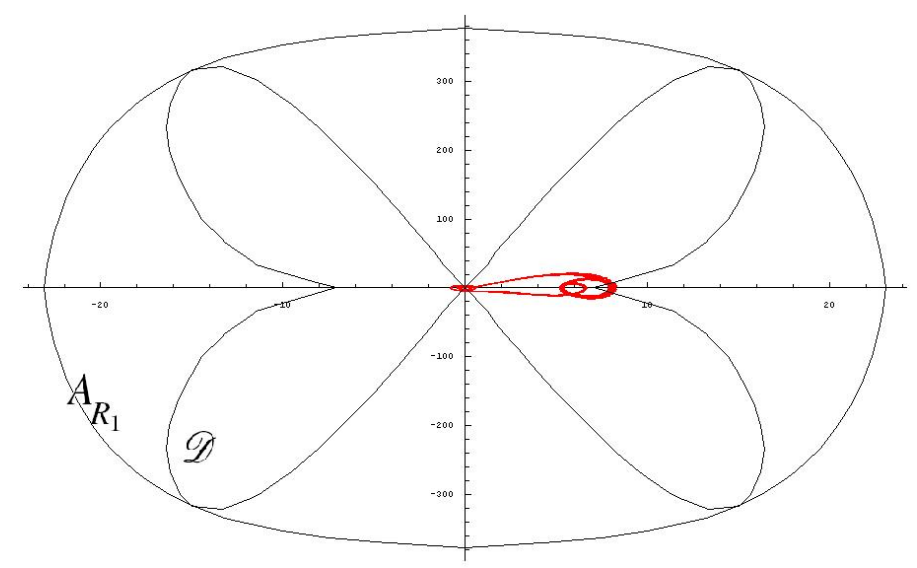

Figura 2.8: Atrator, $\mathcal{D}$, e $A_{R_{1}}$ descritos acima. 

CAPÍtulo

\section{Sistemas lineares}

Seja $A=\left[b_{i j}\right]_{m \times m}$ uma matriz complexa. A norma usual de $A$ é definida por $|A|=$ $\sup _{|v| \neq 0}\left\{\frac{|A v|}{|v|}\right\}=\sup _{0<|v|<1}\left\{\frac{|A v|}{|v|}\right\}=\max _{|v|=1}\{|A v|\}=\inf _{x \in \mathbb{C}^{m}}\{C:|A x| \leq C|x|\}$.

Dizemos que uma sequiência de matrizes $X_{n} \mapsto A$ se $\left|X_{n}-A\right| \mapsto 0$ quando $n \mapsto \infty$ e que $X_{n}$ é uma seqüência limitada se $\left|X_{n}\right|$ for limitada.

Se $A=\left[a_{i j}\right]_{m \times m}$, o polinômio característico de $A$ é $\Phi(\lambda)=\operatorname{det}(\lambda I-A)=\prod_{j=1}^{m}\left(\lambda-\lambda_{j}\right)$, onde os $\lambda_{i}$ são os autovalores de $A$. de $A$.

O conjunto $\sigma(A)=\left\{\lambda_{1}, \ldots, \lambda_{m}\right\}$ é o espectro de $A$ e $r(A)=\max _{\lambda_{i} \in \sigma(A)}\left\{\left|\lambda_{i}\right|\right\}$ é o raio espectral

A multiplicidade algébrica de $\lambda_{i}$ é a multiplicidade de $\lambda_{i}$ como raíz de $\Phi(\lambda)$, e a multiplicidade geométrica de $\lambda_{i}$ é a dimensão do autoespaço $\left\{x \in \mathbb{C}^{m}: A x=\lambda_{i} x\right\}$, isto é, o número de autovetores L.I. associados ao autovalor $\lambda_{i}$.

\subsection{Sistemas lineares de equações de diferenças homogêneas de primeira ordem}

Definição 3.1.1. A forma geral de um sistema linear de equações de diferenças homogêneo e de dimensão mé dada por:

$$
\left\{\begin{array}{ccc}
x_{1}(n+1) & = & a_{11} x_{1}(n)+a_{12} x_{2}(n)+\ldots+a_{1 m} x_{m}(n) \\
x_{2}(n+1) & = & a_{21} x_{1}(n)+a_{22} x_{2}(n)+\ldots+a_{2 m} x_{m}(n) \\
\vdots & \vdots & \vdots \\
x_{m}(n+1) & = & a_{m 1} x_{1}(n)+a_{m 2} x_{2}(n)+\ldots+a_{m m} x_{m}(n)
\end{array}\right.
$$


ou abreviadamente $X^{\prime}=A X$.

Observe que como $X(n+1)=A X(n)$, segue por indução que as soluções do sistema (3.1) são as órbitas do sistema semidinâmico $\Pi\left(n, x_{0}\right)=A^{n} x_{0}$, as quais também podem ser expressas pela seqüência $\left(x_{0}, A x_{0}, A^{2} x_{0}, A^{3} x_{0}, \ldots\right)$, onde $x_{0} \in \mathbb{R}^{m}$. Portanto o problema de calcular as soluções de (3.1) se restringe ao cálculo das potências sucessivas da matriz $A$.

Observe que se $A^{n}$ é uma seqüência convergente com $\lim _{n \rightarrow \infty} A^{n}=B$, então B é uma projeção, isto é $B^{2}=B$. De fato, como $A^{n} \rightarrow B$, temos $\left(A^{n}\right)^{2} \rightarrow B^{2}$ e por continuidade, $A^{2 n} \rightarrow B^{2}$. Como $A^{2 n}$ é subseqüência de $A^{n}$ temos que $A^{2 n} \rightarrow B$ e assim, pela unicidade do limite, $B^{2}=B$.

\subsection{Forma canônica de Jordan}

Se A é uma matriz diagonal, isto é, $A=\operatorname{diag}\left\{\lambda_{1}, \ldots, \lambda_{j}\right\}$, então $A^{n}=\operatorname{diag}\left\{\lambda_{1}^{n}, \ldots, \lambda_{j}^{n}\right\}$.

Definição 3.2.1. Uma matriz quadrada A de ordem n é dita nilpotente se existe r natural não nulo tal que $A^{r}=0$. O menor $r$ com essa propriedade é chamado de índice da matriz A. Temos $n \geq r$.

Exemplo 3.2.1. Um exemplo clássico de matriz nilpotente é dado pela matriz $j \times j$ cuja primeira coluna é o vetor $0 \in \mathbb{R}^{m} e$, para $2 \leq k \leq j$, sua $k$-ésima coluna é $e_{k-1}=$ $(0, \ldots, 0,1,0, \ldots, 0)$, o (k-1)-ésimo elemento da base canônica de $\mathbb{R}^{m}$. Por exemplo, para $j=$ 4, temos:

$$
E=\left[\begin{array}{llll}
0 & 1 & 0 & 0 \\
0 & 0 & 1 & 0 \\
0 & 0 & 0 & 1 \\
0 & 0 & 0 & 0
\end{array}\right], E^{2}=\left[\begin{array}{llll}
0 & 0 & 1 & 0 \\
0 & 0 & 0 & 1 \\
0 & 0 & 0 & 0 \\
0 & 0 & 0 & 0
\end{array}\right], E^{3}=\left[\begin{array}{llll}
0 & 0 & 0 & 1 \\
0 & 0 & 0 & 0 \\
0 & 0 & 0 & 0 \\
0 & 0 & 0 & 0
\end{array}\right], E^{4}=\left[\begin{array}{llll}
0 & 0 & 0 & 0 \\
0 & 0 & 0 & 0 \\
0 & 0 & 0 & 0 \\
0 & 0 & 0 & 0
\end{array}\right]
$$

O próximo teorema pode ser interpretado como uma generalização da fórmula de De Moivre para os números complexos (Veja Lima, [20]).

\section{Teorema 3.2.1. (Decomposição polar)}

Seja E um espaço vetorial de dimensão finita munido de produto interno. Todo operador linear $A: E \rightarrow E$ admite uma decomposição da forma $A=P U$, onde $U: E \rightarrow E$ é ortogonal $e P$ : $E \rightarrow E$ é auto-adjunto não negativo (isto é, todos os seus autovalores são reais não negativos). No caso em que A é inversível, $P$ e $U$ são univocamente determinados, além disso, $P=\sqrt{A A^{*}}$ e $U=P^{-1} A$.

Exemplo 3.2.2. Como aplicação do Teorema da decomposição polar, vamos calcular as potências de $J(\alpha, \beta)=\left[\begin{array}{cc}\alpha & \beta \\ -\beta & \alpha\end{array}\right]$. 
Aplicando o teorema anterior, temos $\left[\begin{array}{cc}\alpha & \beta \\ -\beta & \alpha\end{array}\right]=\left[\begin{array}{ll}\rho & 0 \\ 0 & \rho\end{array}\right]\left[\begin{array}{cc}\cos (\theta) & \operatorname{sen}(\theta) \\ -\operatorname{sen}(\theta) & \cos (\theta)\end{array}\right]$, onde $\rho=$ $\sqrt{\alpha^{2}+\beta^{2}}, \cos (\theta)=\frac{\alpha}{\rho} \operatorname{esen}(\theta)=\frac{\beta}{\rho}$, e por indução:

$\left[\begin{array}{cc}\alpha & \beta \\ -\beta & \alpha\end{array}\right]^{n}=\left[\begin{array}{ll}\rho & 0 \\ 0 & \rho\end{array}\right]^{n}\left[\begin{array}{cc}\cos (\theta) & \operatorname{sen}(\theta) \\ -\operatorname{sen}(\theta) & \cos (\theta)\end{array}\right]^{n}=\left[\begin{array}{cc}\rho^{n} & 0 \\ 0 & \rho^{n}\end{array}\right]\left[\begin{array}{cc}\cos (n \theta) & \operatorname{sen}(n \theta) \\ -\operatorname{sen}(n \theta) & \cos (n \theta)\end{array}\right]$.

O cálculo das potências de uma matriz pode ser simplificado com o seguinte resultado de álgebra linear.

Teorema 3.2.2 (Forma Canônica de Jordan).

a) Caso real: Seja A uma matriz real.

Existe uma matriz real $C$ não singular, tal que $J=C^{-1} A C=\operatorname{diag}\left\{J_{1}, \ldots, J_{k}\right\}$, cada $J_{i} e ́$ da forma $J(\lambda)=\lambda I d+E$ (E como no exemplo 3.2.1), ou $J(\alpha, \beta)$ como no exemplo 3.2.2, onde $\lambda$ é um autovalor real de A e $\alpha+i \beta$ é um autovalor complexo não real de A. A soma das ordens dos blocos de Jordan da forma $J(\lambda)$ é igual a multiplicidade de $\lambda$ como raiz do polinômio característico de A (multiplicidade algébrica de $\lambda$ ) e a quantidade dos blocos $J(\lambda)$ é igual a dimensão do autoespaço associado ao autovalor $\lambda$ (multiplicidade geométrica de $\lambda$ ); a soma das ordens dos blocos de Jordan da forma $J(\alpha, \beta)$ é igual ao dobro da multiplicidade de $\alpha+i \beta$ (ou $\alpha-i \beta$ ) como raíz do polinômio característico de A. A matriz J chama-se Forma canônica real de A e é única, salvo a ordem dos blocos e o sinal da parte imaginária $\beta$ das raízes complexas de A. Duas matrizes reais são similares se e somente se elas têm a mesma forma de Jordan real.

b) Caso complexo: Seja A uma matriz complexa.

Existe uma matriz complexa $C$, não singular, tal que $J=C^{-1} A C=\operatorname{diag}\left\{J_{1}, \ldots, J_{k}\right\}=$ $N+D$,onde $D$ é uma matriz diagonal e $N$ é uma matriz nilpotente tais que $D N=N D$, cada $Q_{i}$ é da forma $J(\lambda)=\lambda I d+E$, onde $\lambda$ é um autovalor complexo de A. A soma das ordens dos blocos de Jordan da forma $J(\lambda)$ é igual a multiplicidade de $\lambda$ como raiz do polinômio característico de A e a quantidade desses blocos é igual a multiplicidade geométrica de $\alpha+i \beta$. A matriz J chama-se Forma canônica complexa de A e é única, salvo a ordem dos blocos. Duas matrizes complexas são similares se e somente se elas têm a mesma forma de Jordan complexa.

Se uma matriz $A=\operatorname{diag}\left\{J_{1}, \ldots, J_{k}\right\}$ está na forma de Jordan, então $A^{n}=\operatorname{diag}\left\{J_{1}^{n}, \ldots, J_{k}^{n}\right\}$. Se $A$ e $B$ são similares com $A=C^{-1} B C$, então $A^{n}=C^{-1} B^{n} C$. Se $\lambda_{i}$ é um autovalor de $A$ e $J\left(\lambda_{i}\right)$ é um bloco de Jordan, então temos: 


$$
J_{i}^{n}=\left[\begin{array}{ccccccc}
\lambda_{0} & 1 & 0 & 0 & \cdots & 0 & 0 \\
0 & \lambda_{0} & 1 & 0 & \cdots & 0 & 0 \\
0 & 0 & \lambda_{0} & 1 & \cdots & 0 & 0 \\
0 & 0 & 0 & \lambda_{0} & \cdots & 0 & 0 \\
\vdots & \vdots & \vdots & \vdots & \ddots & \vdots & \vdots \\
0 & 0 & 0 & 0 & \cdots & \lambda_{0} & 1 \\
0 & 0 & 0 & 0 & \cdots & 0 & \lambda_{0}
\end{array}\right]^{n}=\left[\begin{array}{ccccc}
\lambda_{0}^{n} & n \lambda_{0}^{n-1} & \left(\begin{array}{c}
n \\
2
\end{array}\right) \lambda_{0}^{n-2} & \cdots & \left(\begin{array}{c}
n \\
r-1
\end{array}\right) \lambda_{0}^{n-r} \\
0 & \lambda_{0}^{n} & n \lambda_{0}^{n-1} & \cdots & \left(\begin{array}{c}
n \\
r-2
\end{array}\right) \lambda_{0}^{n-r+1} \\
0 & 0 & \lambda_{0}^{n} & \cdots & \left(\begin{array}{c}
n \\
r-3
\end{array}\right) \lambda_{0}^{n-r+2} \\
\vdots & \vdots & \vdots & \ddots & \vdots \\
0 & 0 & 0 & \cdots & \lambda_{0}^{n}
\end{array}\right]
$$

$A^{n}$ é a matriz solução do sistema, dita matriz de solução principal, e as suas colunas são soluções de (3.1) ditas soluções principais. De fato, se $x_{j}(n)$ é a j-ésima coluna de $A^{n}$ e $x_{j}(n+1)$ é a j-ésima coluna de $A^{n+1}$, temos que $x_{j}(n+1)=A x_{j}(n)$.

Além disso, se $x_{j}$ é uma coluna de $A^{n}$, então $x_{j}$ é a solução de (3.1) satisfazendo $x_{j}(0)=e_{j}$, onde $e_{j}$ é o j-ésimo vetor da base canônica de $\mathbb{R}^{m}$. Isso segue do fato que $A^{0}=I d$.

Proposição 3.2.1. O conjunto $\varphi$ de todas as soluções $x: \mathbb{N} \times \mathbb{R}^{m} \mapsto \mathbb{R}^{m}$ de (3.1) é um espaço vetorial sobre $\mathbb{R}$ de dimensão $m$, e as soluções principais constituem uma base para esse espaço vetorial.

\section{Prova:}

É claro que a seqüência nula é solução de (3.1).

Se $\varphi$ e $\psi$ são soluções de (3.1), então $\varphi^{\prime}=A \varphi$ e $\psi^{\prime}=A \psi$.

Se $a, b$ são constantes arbitrárias, então $(a \varphi)^{\prime}=a \varphi^{\prime}=a A \varphi=A a \varphi=A(a \varphi)$ e $(b \psi)^{\prime}=b \psi^{\prime}=$ $b A \psi=A b \psi=A(b \psi)$.

Logo, se $\gamma=a \varphi+b \psi, \gamma^{\prime}=(a \varphi+b \psi)^{\prime}=(a \varphi)^{\prime}+(b \psi)^{\prime}=a \varphi^{\prime}+b \psi^{\prime}=a A \varphi+b A \psi=A a \varphi+$ $A b \psi=A(a \varphi+b \psi)=A \gamma$.

Com isso fica provado que o conjunto das soluções de (3.1) é um espaço vetorial.

Já foi visto que se $x_{j}(n)$ é a j-ésima coluna de $A^{n}$, então $x_{j}$ é a solução de (3.1) tal que $x_{j}(0)=e_{j}$, com isso temos que que o conjunto $\left\{x_{1}(n), \ldots, x_{m}(n)\right\}$ constituído pelas colunas de $A^{n}$ é linearmente independente, resta apenas mostrar que ele é um conjunto gerador do espaço vetorial das soluções de (3.1), e com isso conclui-se que a dimensão desse espaço vetorial é m.

Seja $\varphi$ a solução de (3.1) tal que $\varphi(0)=\left[\begin{array}{c}a_{1} \\ \vdots \\ a_{m}\end{array}\right]$.

Temos que $\varphi(0)=a_{1} x_{1}(0)+\ldots+a_{m} x_{m}(0)$, e por indução, $\varphi(n)=a_{1} x_{1}(n)+\ldots+a_{m} x_{m}(n)$ 
para todo n natural. Isso mostra que qualquer solução de (3.1) é combinação linear das soluções principais desse sistema e a prova está concluída.

Note que se $\left\langle v_{1}, \cdots v_{m}\right\rangle$ é uma base de autovetores de $A$ associados aos autovalores $\lambda_{1}, \cdots, \lambda_{m}$ respectivamente, então a solução geral de (3.1) é dada por $x(n)=a_{1} \lambda_{1}^{n} v_{1}+\cdots+a_{m} \lambda_{m}^{n} v_{m}$.

Proposição 3.2.2. A estabilidade assintótica da origem (com relação a 3.1) é sempre global e são equivalentes:

1. $A^{n} \rightarrow 0$ quando $n \rightarrow \infty$.

2. Todas as soluções se aproximam da origem quando $n \rightarrow \infty$, isto é, a origem é globalmente atrativa.

3. A origem é globalmente assintoticamente estável.

4. Todos os autovalores $\lambda$ de A satisfazem $|\lambda|<1$, isto é, $r(A)<1$.

\section{Prova:}

$$
\text { 1. } \Rightarrow 2 \text {. }
$$

Como as soluções de (3.1) são da forma $\left(v, A v, A^{2} v, A^{3} v, \ldots\right)$ e $A^{n} \rightarrow 0$, seque que toda solução se aproxima da origem quando $n \rightarrow \infty$.

2. $\Rightarrow 3$.

Considere $\mathbb{R}^{m}$ com a norma da soma, isto é, se $x=\left(x_{1}, \cdots, x_{m}\right) \in \mathbb{R}^{m}$ então $|x|=\sum_{i=1}^{m}\left|x_{i}\right|$.

Como $A^{n} x \rightarrow 0$ para todo $x \in \mathbb{R}^{m}$, em particular temos que $A^{n} e_{i} \rightarrow 0$, onde $e_{i}$ é o i-ésimo vetor da base canônica de $\mathbb{R}^{m}$. Segue que existe $N$ tal que $\left|A^{n} e_{i}\right| \leq 1$ para todo $n \geq N$ e existe $M_{i}$ tal que $\left|A^{n} e_{i}\right| \leq M_{i}$ para todo $n \in \mathbb{N}$, onde $M_{i}=\max _{1 \leq n<N}\left\{1,\left|A^{n} e_{i}\right|\right\}$.

Seja $M=\max _{1 \leq i \leq m}\left\{M_{i}\right\}$.

Seja $x=\left(x_{1}, \cdots, x_{m}\right)$ tal que $|x| \leq 1$. Como $x=\sum_{i=1}^{m} x_{i} e_{i}$, temos $\left|A^{n} x\right|=\left|\sum_{i=1}^{m} x_{i} A^{n} e_{i}\right| \leq$ $\sum_{i=1}^{m}\left|x_{i} A^{n} e_{i}\right|=\sum_{i=1}^{m}\left|x_{i}\right|\left|A^{n} e_{i}\right| \leq M \sum_{i=1}^{m}\left|x_{i}\right|=M|x| \leq M$.

Assim, provamos que se $|x| \leq 1$, então $A^{n} x \leq M$ para todo $n \in \mathbb{N}$.

Agora vamos verificar a estabilidade assintótica da origem. Dado varepsilon $>0$ e tomando $\delta=\frac{\varepsilon}{M}$, então se $|x|<\delta, \frac{|x|}{\delta}<1$ e disso segue que $\left|A^{n} \frac{x}{\delta}\right| \leq M$, donde obtemos que $\left|A^{n} x\right| \leq M \delta<\varepsilon$.

\section{3. $\Rightarrow 4$.}

Suponha que existe um autovalor $\lambda$ de $A$ tal que $|\lambda| \geq 1$, e seja $v$ é um autovetor correspondente a $\lambda$ com $|v|=1$ temos: $\left|A^{n} v\right|=\left|\lambda^{n} v\right|=\left|\lambda^{n}\right||v|=|\lambda|^{n} \geq 1$. Isso mostra que $A^{n} \nrightarrow 0$ quando $n \rightarrow \infty$. 
4. $\Rightarrow 1$.

Para provar essa implicação, faremos uso do Teorema de Holmes (Veja Gabriel [8]):

Teorema 3.2.3 (Holmes).

$\operatorname{Seja}(X,||$.$) um espaço de Banach. Dados A \in \mathcal{L}(X)$ e $\varepsilon>0$, existe uma norma $\|.\|_{\varepsilon}$ equivalente $a|$.$| , tal que r(A) \leq\|A\|_{\varepsilon} \leq r(A)+\varepsilon$.

Como por hipótese $r(A)<1$, dado $\varepsilon>0$ tal que $r(A)+\varepsilon<1$, pelo Teorema de Holmes existe $\|$. $\|_{\varepsilon}$ tal que $\|A\|_{\varepsilon} \leq r(A)+\varepsilon<1$ (Lembre que todas as normas em $\mathbb{R}^{m}$ são equivalentes).

Assim, $\left\|A^{n}\right\|_{\varepsilon} \leq\|A\|_{\varepsilon}^{n} \rightarrow 0$ quando $n \rightarrow \infty$.

Se a matriz A cumpre uma das condições da Proposição 3.2.2 (e portanto cumpre todas) então ela é dita estável.

$r(A) \leq 1$ é uma condição necessária para que $A$ seja estável, mas essa condição não é suficiente como mostra o seguinte exemplo:

Exemplo 3.2.3. Considere o sistema

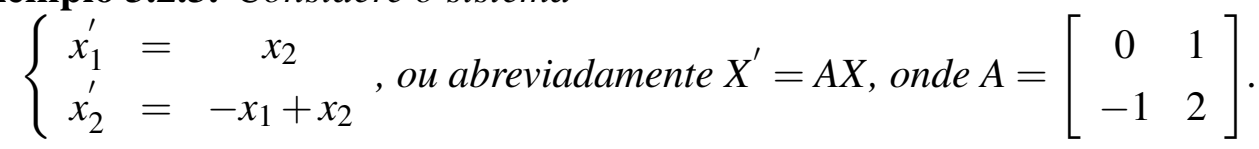

$O$ polinômio característico de A é $p(x)=x^{2}-2 x+1=(x-1)^{2}$, donde $r(A)=1$, mas $A^{n}=\left[\begin{array}{cc}-n+1 & n \\ -n & n+1\end{array}\right]$. Logo $A^{n} \nrightarrow 0$ quando $n \rightarrow \infty$ e portanto A não é estável.

\subsection{Critério de Schur-Cohn}

Nessa seção apresentaremos um critério de Schur-Cohn que é um algoritmo computacional que decide se uma dada matriz é estável ou não. (Para uma referência, veja Henrici, [14], veja também LaSalle, [18]).

Definição 3.3.1. Os internos de uma matriz quadrada são a própria matriz e as matrizes obtidas desta, eliminando-se sucessivamente a primeira e última linha e a primeira e última coluna.

Para a matriz $[A]_{5 \times 5}, A=\left[\begin{array}{lllll}a_{11} & a_{12} & a_{13} & a_{14} & a_{15} \\ a_{21} & a_{22} & a_{23} & a_{24} & a_{25} \\ a_{31} & a_{32} & a_{33} & a_{34} & a_{35} \\ a_{41} & a_{42} & a_{43} & a_{44} & a_{45} \\ a_{51} & a_{52} & a_{53} & a_{54} & a_{55}\end{array}\right]$, os internos de $A$ são a própria
matriz $A,\left[\begin{array}{lll}a_{22} & a_{23} & a_{24} \\ a_{32} & a_{33} & a_{34} \\ a_{42} & a_{43} & a_{44}\end{array}\right] \mathrm{e}\left[a_{33}\right]$. 
Para a matriz $[A]_{6 \times 6}, A=\left[\begin{array}{llllll}a_{11} & a_{12} & a_{13} & a_{14} & a_{15} & a_{16} \\ a_{21} & a_{22} & a_{23} & a_{24} & a_{25} & a_{26} \\ a_{31} & a_{32} & a_{33} & a_{34} & a_{35} & a_{36} \\ a_{41} & a_{42} & a_{43} & a_{44} & a_{45} & a_{46} \\ a_{51} & a_{52} & a_{53} & a_{54} & a_{55} & a_{56} \\ a_{61} & a_{62} & a_{63} & a_{64} & a_{65} & a_{66}\end{array}\right]$, os internos de $A$ são a própria
matriz $A,\left[\begin{array}{llll}a_{22} & a_{23} & a_{24} & a_{25} \\ a_{32} & a_{33} & a_{34} & a_{35} \\ a_{42} & a_{43} & a_{44} & a_{45} \\ a_{52} & a_{53} & a_{54} & a_{55}\end{array}\right] \mathrm{e}\left[\begin{array}{lll}a_{33} & a_{34} \\ a_{43} & a_{44}\end{array}\right]$.

\section{Proposição 3.3.1. (Critério de Schur-Cohn)}

Uma condição necessária e suficiente para que um polinômio $p(x)=a_{m} x^{m}+a_{m-1} x^{m-1}+$ $\ldots+a_{0}$ de coeficientes reais tenha todas as raízes contidas em $B_{1}(0)$ é:

a) $p(1)>0 e(-1)^{m} p(-1)>0$.

b) As matrizes seguintes têm internos positivos

$$
M_{ \pm}=\left[\begin{array}{cccccc}
a_{m} & 0 & 0 & \cdots & 0 & 0 \\
a_{m-1} & a_{m} & 0 & \cdots & 0 & 0 \\
a_{m-2} & a_{m-1} & a_{m} & \cdots & 0 & 0 \\
\vdots & \vdots & \vdots & \ddots & \vdots & \vdots \\
a_{3} & a_{4} & a_{5} & \cdots & a_{m} & 0 \\
a_{2} & a_{3} & a_{4} & \cdots & a_{m-1} & a_{m}
\end{array}\right] \pm\left[\begin{array}{cccccc}
0 & 0 & \cdots & 0 & 0 & a_{0} \\
0 & 0 & \cdots & 0 & a_{0} & a_{1} \\
0 & 0 & \cdots & a_{0} & a_{1} & a_{2} \\
\vdots & \vdots & \ddots & \vdots & \vdots & \vdots \\
0 & a_{0} & \cdots & a_{m-5} & a_{m-4} & a_{m-3} \\
a_{0} & a_{1} & \cdots & a_{m-4} & a_{m-3} & a_{m-2}
\end{array}\right]
$$

\subsection{Um funcional de Liapunov para $X^{\prime}=A X$}

Nesta seção vamos nos restringir as matrizes reais.

Definição 3.4.1. Os menores principais de uma matriz quadrada A de ordem n são as matrizes

$$
A_{k}:=\left[\begin{array}{ccc}
a_{11} & \cdots & a_{1 k} \\
\vdots & & \vdots \\
a_{k 1} & \cdots & a_{k k}
\end{array}\right], \quad k=1, \cdots, n .
$$

Definição 3.4.2. Uma matriz quadrada real $A$ de ordem $m$ é definida positiva se a forma quadrática $V(x)=x^{t} A x=\sum_{i, j=1}^{m} a_{i j} x_{i} x_{j}$ for definida positiva. 
Como $V(x)=\frac{1}{2} x^{t}\left(A+A^{t}\right) x$, segue que $A$ é definida positiva se e somente se $A+A^{t}$, (que é uma matriz simétrica) também o for. Desta observação conclui-se que é suficiente trabalharmos com matrizes simétricas.

Uma das razões para trabalharmos com matrizes simétricas é o:

Proposição 3.4.1 (Critério de Silvester).

Uma matriz simétrica real A é definida positiva se e somente se os determinantes dos seus menores principais são positivos, isto é, se e somente se

$$
a_{11}>0,\left|\begin{array}{ll}
a_{11} & a_{12} \\
a_{21} & a_{22}
\end{array}\right|>0,\left|\begin{array}{lll}
a_{11} & a_{12} & a_{13} \\
a_{21} & a_{22} & a_{23} \\
a_{31} & a_{32} & a_{33}
\end{array}\right|>0, \ldots, \operatorname{det}(A)>0 .
$$

Uma prova desse resultado pode ser encontrada em Hoffman \& Kunze, [16].

O critério de silvester não é válido se retirarmos a hipótese da matriz $A$ ser simétrica, por exemplo, se $A=\left[\begin{array}{cc}1 & 0 \\ -4 & 1\end{array}\right]$, então $V(x, y)=x^{t} A x=x^{2}-4 x y+y^{2}$.

Agora utilizaremos o método direto de Liapunov para obter uma caracterização das matrizes estáveis. Retornando a equação (3.1) e considerando um funcional da forma $V(x)=x^{t} B x$, onde $B$ é definida positiva, temos $\dot{V}(x)=V(T(x))-V(x)=V(A x)-V(x)=(A x)^{t} B(A x)-x^{t} B x=$ $x^{t} A^{t} B A x-x^{t} B x=x^{t}\left(A^{t} B A-B\right) x$.

Se $A^{t} B A-B$ é definida negativa, pelo teorema de estabilidade assintótica de Liapunov, a origem é assintóticamente estável. Reciprocamente, suponha $A$ estável e considere a equação

$$
A^{t} B A-B=-C
$$

onde $C$ é uma matriz dada. Se essa equação tem uma solução, então $\left(A^{t}\right)^{k+1} B A^{k+1}-\left(A^{t}\right)^{k} B A^{k}=$ $-\left(A^{t}\right)^{k} C A^{k}$.

Somando as parcelas para $k$ variando entre 0 e $n$, temos $\left(A^{t}\right)^{n+1} B A^{n+1}-B=-\sum_{k=0}^{n}\left(A^{t}\right)^{k} C A^{k}$.

Tomando $n \rightarrow \infty$ obtemos $B=\sum_{k=0}^{\infty}\left(A^{t}\right)^{k} C A^{k}$, esta série é convergente pois como $A$ é uma matriz estável, então $r\left(A^{t}\right)=r(A)<1$, assim existe $\varepsilon>0$ tal que $r\left(A^{t}\right)+\varepsilon=r(A)+\varepsilon<1$, e pelo Teorema de Holmes concluimos que existe \|\|$_{\varepsilon}$ equivalente a || tal que $r(A)<\|A\|_{\varepsilon}<$ $r(A)+\varepsilon<1$ e $r\left(A^{t}\right)<\left\|A^{t}\right\|_{\varepsilon}<r\left(A^{t}\right)+\varepsilon<1$. Logo:

$$
\begin{aligned}
& \|B\|_{\varepsilon}=\left\|\sum_{k=0}^{\infty}\left(A^{t}\right)^{k} C A^{k}\right\|_{\varepsilon} \leq \sum_{k=0}^{\infty}\left\|\left(A^{t}\right)^{k} C A^{k}\right\|_{\varepsilon} \leq \sum_{k=0}^{\infty}\left\|\left(A^{t}\right)^{k}\right\|\left\|_{\varepsilon}\right\| C\left\|_{\varepsilon}\right\| A^{k} \|_{\varepsilon} \leq \\
& \sum_{k=0}^{\infty}\left\|A^{t}\right\|_{\varepsilon}^{k}\|C\|_{\varepsilon}\|A\|_{\varepsilon}^{k} \leq \sum_{k=0}^{\infty}\|C\|_{\varepsilon}\left(r^{2}\right)^{k}<\infty \text {, onde } r=\max \left\{\|A\|_{\varepsilon},\left\|A^{t}\right\|_{\varepsilon}\right\}<1 \text {. Assim, a } \\
& \text { série acima converge na norma original, e de fato } B \text { é uma solução de (3.3). }
\end{aligned}
$$




\section{Teorema 3.4.1 (Liapunov).}

Se existem matrizes B e C positivas definidas satisfazendo (3.3), então A é estável.

Reciprocamente, Se A é uma matriz estável, então dada uma matriz $C$ a equação (3.3) tem uma única solução; se $C$ é definida positiva então $B$ também é; e se $C$ é simétrica então $B$ também é simétrica.

Corolário 3.4.1. Se $r(A) \geq 1$ e a equação (3.3) tem uma solução $B$ para alguma matriz $C$ definida positiva, então ou $B$ é semidefinida negativa ou $B$ é indefinida, isto é, $x^{t} B x$ é negativo para algum $x$.

Consideraremos agora a questão da unicidade da solução da equação 3.3, mais geralmente, considere a equação

$$
A_{1} X A_{2}-X=C
$$

onde $A_{1}$ é uma matriz $m \times m, A_{2}$ é uma matriz $n \times n, X$ e $C$ são matrizes $m \times n$.

Proposição 3.4.2. A equação (3.4) tem uma única solução se e somente se nenhum autovalor de $A_{1}$ é inverso multiplicativo de um autovalor de $A_{2}$.

\section{Prova:}

A condição "a equação (3.4) tem uma única solução" é equivalente a " $A_{1} X A_{2}=X$ implica que $X=0$ "'.

Assuma então que essa condição em $A_{1}$ e $A_{2}$ é satisfeita. Da igualdade $A_{1} X A_{2}=X$ segue que $X=A_{1} X A_{2}=A_{1}^{2} X A_{2}^{2}=A_{1}^{3} X A_{2}^{3}=\cdots$ e que $A_{1}^{j} X=A_{1}^{k} X A_{2}^{k-j}$ para $k \geq j \geq 0$.

Defina os polinômios $p(\lambda)=\sum_{j=0}^{k} a_{j} \lambda^{j}$ e $p^{*}(\lambda)=\sum_{j=0}^{k} a_{j} \lambda^{k-j}=\lambda^{k} p\left(\frac{1}{\lambda}\right)$.

Seja $\phi_{i}$ o polinômio característico de $A_{i}$. Como $\phi_{1}(\lambda)$ e $\phi_{2}^{*}(\lambda)$ são relativamente primos pois nenhum autovalor de $A_{1}$ é inverso multiplicativo de um autovalor de $A_{2}$, pelo algoritmo de Euclides existem polinômios $p(\lambda)$ e $q(\lambda)$ tais que $p(\lambda) \phi_{1}(\lambda)+q(\lambda) \phi_{2}^{*}(\lambda)=1$.

Definindo $\phi(\lambda)=q(\lambda) \phi_{2}^{*}(\lambda)$ e notando que $\phi^{*}(\lambda)=q^{*}(\lambda) \phi_{2}(\lambda)$, pelo teorema de CayleyHamilton temos $\phi^{*}\left(A_{2}\right)=0$ e $\phi\left(A_{1}\right)=I d$, segue que $A_{1} X A_{2}=X$ implica $X=0$.

Para provar a recíproca, assuma que $\lambda$ é um autovalor de $A_{1}$ e $\lambda^{-1}$ é um autovalor de $A_{2}$ (e de $A_{2}^{t}$ também). Sejam $x_{1}^{t}=\left(u_{1}, \cdots, u_{m}\right)^{t}$ e $x_{2}^{t}=\left(v_{1}, \cdots, v_{n}\right)^{t}$ com $x_{1} \neq 0$ e $x_{2} \neq 0$ e tais que $A_{1} x_{1}=\lambda x_{1}$ e $A_{2}^{t}=\lambda^{-1} x_{2}$.

Definindo $X:=\left[\begin{array}{ccc}u_{1} v_{1} & \cdots & u_{1} v_{n} \\ \vdots & \ddots & \vdots \\ u_{m} v_{1} & \cdots & u_{m} v_{n}\end{array}\right]$ Temos que $X \neq 0$ e $A_{1} X A_{2}=X$. 
Se $A$ é estável, então nenhum recíproco multiplicativo de um autovalor de $A$ é um autovalor de $A$, e esta proposição fornece uma forma de mostrar que a equação (3.4) tem uma única solução $B$ para cada $C$ se $A$ é estável.

\subsection{Estabilidade por aproximação linear}

Nesta seção determinaremos a estabilidade da equação não linear

$$
x^{\prime}=A x+f(x),
$$

onde $f(x)$ é $o(x)$ quando $|x| \rightarrow 0$, isto é, $f(x)$ é tal que dado $\varepsilon>0$ existe um $\delta>0$ tal que $|f(x)|<\varepsilon|x|$ para todo $|x|<\delta$.

Teorema 3.5.1. Assuma que $f(x)$ é o(x) quando $|x| \rightarrow 0$. Se A é uma matriz estável, então a origem é um ponto de equilíbrio assintoticamente estável para (3.5). Se $r(A)>1$, então a origem é um ponto de equilíbrio instável para (3.5).

\section{Prova:}

assuma $A$ estável e $C=I d$. Temos que a equação (3.3) tem uma solução $B$ que é (simétrica e) definida positiva. Então se $V(x)=x^{t} B x$, temos que

$$
\begin{gathered}
\dot{V}(x)=V(T(x))-V(x)= \\
V(A x+f(x))-V(x)= \\
(A x+f(x))^{t} B(A x+f(x))-x^{t} B x= \\
\left(x^{t} A^{t}+f(x)^{t}\right) B(A x+f(x))-x^{t} B x= \\
x^{t} A^{t} B A x+x^{t} A^{t} B f(x)+f(x)^{t} B A x+f(x)^{t} B f(x)-x^{t} B x= \\
x^{t}\left(A^{t} B A-B\right) x+x^{t} A^{t} B f(x)+f(x)^{t} B A x+f(x)^{t} B f(x)= \\
x^{t}(-I d) x+x^{t} A^{t} B f(x)+\left(x^{t} A^{t} B f(x)\right)^{t}+V(f(x))= \\
-x^{t} x+\left[2 x^{t} A^{t} B f(x)+V(f(x))\right]
\end{gathered}
$$

Temos que $2 x^{t} A^{t} B f(x)+V(f(x))$ é $o\left(x^{2}\right)$ em uma vizinhança da origem, logo existe $\varepsilon>0$ tal que $\dot{V}(x)<0$ em $B_{\varepsilon}(0)-\{0\}$ e $\dot{V}(0)=0$. Segue do Teorema de estabilidade assintótica de Liapunov que a origem é assintoticamente estável.

Se $r(A)>1$, e tomando $\beta>0$ tal que nenhum autovalor de $A_{0}=(1+\beta)^{\frac{1}{2}} A$ é autovalor de $A$ e suficientemente pequeno que $r\left(A_{0}\right)>1$; (temos $\left.(1+\beta)^{-\frac{1}{2}}<r(A)\right)$.

Então pela proposição 3.4 .2 e pelo corolário 3.4.1, existe uma matriz $B$ satisfazendo 


$$
\begin{gathered}
A_{0}^{t} B A_{0}-B=-I d \Rightarrow \\
\left((1+\beta)^{-\frac{1}{2}} A\right)^{t} B\left((1+\beta)^{-\frac{1}{2}} A\right)-B=-I d \Rightarrow \\
(1+\beta)^{-1} A^{t} B A-B=-I d \Rightarrow \\
\left.A^{t} B A-(1+\beta) B=-(1+\beta)\right) I d \Rightarrow \\
A^{t} B A-B=\beta B-(1+\beta) I d,
\end{gathered}
$$

onde $V(x)=-x^{t} B x$ toma valores positivos para algum $x$. Temos (com relação a 3.5):

$$
\begin{gathered}
\dot{V}(x)=-(A x+f(x))^{t} B(A x+f(x))+x^{t} B x= \\
-\left(x^{t} A^{t}+f(x)^{t}\right)(B A x+B f(x))+x^{t} B x= \\
-x^{t} A^{t}(B A x+B f(x))-f(x)^{t}(B A x+B f(x))+x^{t} B x= \\
-x^{t} A^{t} B A x-x^{t} A^{t} B f(x)-f(x)^{t} B A x-f(x)^{t} B f(x)+x^{t} B x= \\
x^{t}\left(-A^{t} B A+B\right) x-x^{t} A^{t} B f(x)-\left(x^{t} A^{t} B^{t} f(x)\right)^{t}+f(x)^{t} B f(x)= \\
x^{t}\left(-A^{t} B A+B\right) x-x^{t} A^{t} B f(x)-\left(x^{t} A^{t} B f(x)\right)^{t}+f(x)^{t} B f(x)= \\
x^{t}(-\beta B+(1+\beta) I d) x-2 x^{t} A^{t} B f(x)+V(f(x))= \\
-\beta x^{t} B x+x^{t}(1+\beta) x-2 x^{t} A^{t} B f(x)+V(f(x))= \\
\beta V(x)+x^{t}(1+\beta) x+2 x^{t} A^{t} B f(x)+V(f(x))
\end{gathered}
$$

Temos que $2 x^{t} A^{t} B f(x)+V(f(x))$ é $o\left(x^{2}\right)$ numa vizinhança da origem. Logo, para algum $0<\alpha<1$ e $\delta$ suficientemente pequeno, $W(x)=x^{t}(1+\beta) x+2 x^{t} A^{t} B f(x)+V(f(x)) \geq \alpha x^{t} x$ para todo $|x|<\delta$. Pela proposição 2.1.6, temos que a origem é instável para 3.5.

\subsection{Equações de diferenças lineares de ordem $m$}

Uma equação da forma

$$
x^{(m)}+a_{m-1} x^{(m-1)}+\ldots+a_{2} x^{\prime \prime}+a_{1} x^{\prime}+a_{0} x=0
$$

chama-se equação de diferenças linear homogênea de ordem $m$.

Observação 3.6.1. A equação (3.6) é equivalente ao sistema:

$$
\left[\begin{array}{c}
x \\
x^{\prime} \\
x^{\prime \prime} \\
\vdots \\
x^{m-2} \\
x^{m-1}
\end{array}\right]^{\prime}=\underbrace{\left[\begin{array}{cccccc}
0 & 1 & 0 & 0 & \cdots & 0 \\
0 & 0 & 1 & 0 & \cdots & 0 \\
0 & 0 & 0 & 1 & \cdots & 0 \\
\vdots & \vdots & \vdots & \vdots & \ddots & \vdots \\
0 & 0 & 0 & 0 & \cdots & 1 \\
-a_{0} & -a_{1} & -a_{2} & -a_{3} & \cdots & -a_{m-1}
\end{array}\right]}_{A} \underbrace{\left[\begin{array}{c}
x \\
x^{\prime} \\
x^{\prime \prime} \\
\vdots \\
x^{m-2} \\
x^{m-1}
\end{array}\right]}_{X}
$$

Ou abreviadamente $X^{\prime}=A X$. 
$O$ vetor $X(n)$ é o estado de (3.6) no tempo $n-m+1$.

Se $x(n)$ é uma solução de (3.7) então $y(n)=x_{1}(n)$ é uma solução de (3.6). Reciprocamente, se y(n) é solução de (3.6), então $\left[\begin{array}{c}y(n) \\ y^{\prime}(n) \\ y^{\prime \prime}(n) \\ \vdots \\ y^{m-2}(n) \\ y^{m-1}(n)\end{array}\right]$ é solução de (3.7).

A matriz $A_{0}$ é dita matriz compnheira principal de $A_{0}$.

Proposição 3.6.1. O conjunto $\varphi$ de todas as soluções de (3.6) é um espaço vetorial sobre $\mathbb{R}$ cuja dimensão é igual a ordem dessa equação $(\mathrm{m})$.

\section{Prova:}

A prova dessa proposição segue da Proposição 3.2.1 e da observação 3.6.1.

\subsection{Soluções de uma equação de m-ésima ordem}

Procuraremos inicialmente soluções da forma $\lambda^{n} \operatorname{com} n \in \mathbb{N}$ (Se $\lambda=0$, convencionaremos que $0^{0}=1$ ) para (3.6).

$$
\begin{gathered}
a_{m}\left(\lambda^{n}\right)^{(m)}+a_{m-1}\left(\lambda^{n}\right)^{(m-1)}+\ldots+a_{2}\left(\lambda^{n}\right)^{\prime \prime}+a_{1}\left(\lambda^{n}\right)^{\prime}+a_{0}\left(\lambda^{n}\right)= \\
a_{m} \lambda^{n+m}+a_{m-1} \lambda^{n+m-1}+\ldots+a_{2} \lambda^{n+2}+a_{1} \lambda^{n+1}+a_{0} \lambda^{n}= \\
\lambda^{n}\left(a_{m} \lambda^{m}+a_{m-1} \lambda^{m-1}+\ldots+a_{2} \lambda^{2}+a_{1} \lambda+a_{0}\right)
\end{gathered}
$$

Assim, $\left(\lambda^{n}\right)$ é uma solução de (3.6) se e somente se $\lambda$ é raíz de $a_{m} x^{m}+a_{m-1} x^{m-1}+\ldots+$ $a_{2} x^{2}+a_{1} x+a_{0}=0$. Este polinômio é denominado equação caraceterística, ou polinômio característico de (3.6).

Se toda solução de (3.6) é da forma $x(n)=c_{1} \lambda_{1}^{n}+\ldots+c_{m} \lambda_{m}^{n}$ e é unicamente determinada pelas condições iniciais $y(0)=b_{1}, y(1)=b_{2}, \ldots, y(m-1)=b_{m}$. Temos então o seguinte sistema linear:

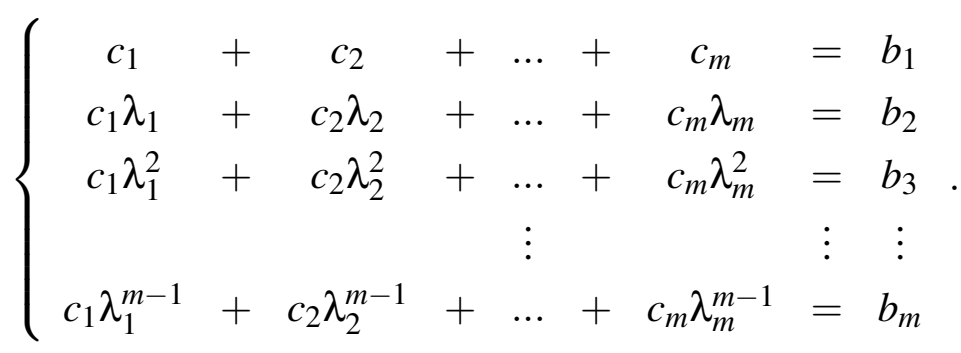


Assim, $\Delta\left(\lambda_{1}, \ldots, \lambda_{m}\right)=\left|\begin{array}{cccc}1 & 1 & \ldots & 1 \\ \lambda_{1} & \lambda_{2} & \ldots & \lambda_{m} \\ \lambda_{1}^{2} & \lambda_{2}^{2} & \ldots & \lambda_{m}^{2} \\ \vdots & \vdots & \ddots & \vdots \\ \lambda_{1}^{m-1} & \lambda_{2}^{m-1} & \ldots & \lambda_{m}^{m-1}\end{array}\right| \neq 0$.

$\Delta\left(\lambda_{1}, \ldots, \lambda_{m}\right)$ é um determinante de Vandermonde; verifica-se que $\Delta\left(\lambda_{1}, \ldots, \lambda_{m}\right)=$ $\prod_{1 \leq i \leq j \leq m}\left(\lambda_{j}-\lambda_{i}\right)$. Disso segue que se a equação característica de (3.6): $a_{m} x^{m}+a_{m-1} x^{m-1}+$ $\ldots+a_{2} x^{2}+a_{1} x+a_{0}=\left(x-\lambda_{1}\right)\left(x-\lambda_{2}\right) \ldots\left(x-\lambda_{m}\right)$, onde $\lambda_{i} \neq \lambda_{j}$ se $i \neq j$, então a solução geral de (3.6) é $y(n)=c_{1} \lambda_{1}^{n}+c_{2} \lambda_{2}^{n}+\ldots+c_{m} \lambda_{m}^{n}$, e reciprocamente, se a solução geral de (3.6) é dessa forma, então os $\lambda_{i}$ são distintos.

No caso de raízes múltiplas, isto é, se $x^{m}+a_{m-1} x^{m-1}+\ldots+a_{2} x^{2}+a_{1} x+a_{0}=\left(x-\lambda_{1}\right)^{s_{1}}(x-$ $\left.\lambda_{2}\right)^{s_{2}} \ldots\left(x-\lambda_{r}\right)^{s_{r}}$, então $\left(\begin{array}{c}n \\ j\end{array}\right) \lambda_{i}^{n-j}$, onde $0 \leq j \leq s_{i}-1$ e $1 \leq i \leq r$, constituem uma base de soluções para (3.6).

Exemplo 3.7.1 (Sequiência de Fibonacci).

Considere o PVI $\left\{\begin{array}{l}y^{\prime \prime}-y^{\prime}-y=0 \\ y(0)=0 \\ y^{\prime}(0)=1\end{array}\right.$

Seu polinômio característico é $x^{2}-x-1$, logo a solução geral é $c_{1}\left(\frac{1+\sqrt{5}}{2}\right)^{n}+c_{2}\left(\frac{1-\sqrt{5}}{2}\right)^{n}$ e através de alguns cálculos simples, obtemos que a solução do PVI é:

$$
y(n)=\frac{1}{\sqrt{5}}\left(\frac{1+\sqrt{5}}{2}\right)^{n}-\frac{1}{\sqrt{5}}\left(\frac{1-\sqrt{5}}{2}\right)^{n}
$$

\subsection{Fórmula da variação das constantes}

Proposição 3.8.1 (Princípio da superposição).

Se $x_{1}(n)$ e $x_{2}(n)$ satisfazem $x_{1}^{\prime}=A x_{1}+f_{1}(n)$ e $x_{2}^{\prime}=A x_{2}+f_{2}(n)$, então $x(n)=c_{1} x_{1}(n)+$ $c_{2} x_{2}(n)$ é solução de $x^{\prime}=A x_{1}+c_{1} f_{1}(n)+c_{2} f_{2}(n)$.

Do princípio da superposição, temos que se $x_{1}(n)$ e $x_{2}(n)$ são soluções de $x^{\prime}=A x+f(n)$ então $x_{1}(n)-x_{2}(n)$ é solução da equação homogênea $x^{\prime}=A x$, assim, se $x_{1}(n)$ é uma solução de $x^{\prime}=A x+f(n)$ então a solução geral de $x^{\prime}=A x+f(n)$ é $A^{n} v+x_{1}(n)$. Noutras palavras, a solução geral de $x^{\prime}=A x+f(n)$ é a solução geral de $x^{\prime}=A x$ adicionada a uma solução particular de $x^{\prime}=A x+f(n)$.

Proposição 3.8.2 (Fórmula da variação das constantes).

Via indução finita, temos que a solução de $x^{\prime}=A x+f(n)$ satisfazendo $x(0)=x_{0}$ é dada por $x(n)=A^{n} x(0)+\sum_{k=0}^{n-1} A^{n-k-1} f(k)$, ou ainda $x(n)=A^{n} x(0)+\sum_{k=0}^{n-1} A^{n} f(n-k-1)$. 


\subsection{Matrizes companheiras}

Considere a equação de diferenças de m-ésima ordem (3.6): $x^{(m)}+a_{m-1} x^{(m-1)}+\ldots+a_{2} x^{\prime \prime}+$ $a_{1} x^{\prime}+a_{0} x=0, \operatorname{dados} y(0), y^{\prime}(0)=y(1), y^{\prime \prime}(0)=y(2), \ldots, y^{(m-1)}(0)=y(m-1)$

Definição 3.9.1. Uma matriz $A_{0}$ é uma matriz companheira da equação de diferenças (3.6), se for similar a sua matriz companheira principal.

Se A é uma matriz companheira de (3.6) então o polinômio característico de A é igual ao polinômio característico de (3.6).

Nem toda matriz é uma matriz companheira de alguma equação de diferenças de $m$-ésima ordem (a matriz identidade por exemplo), noutras palavras, nem todo sistema $X^{\prime}=A X$ é equivalente a uma equação de diferenças de $m$-ésima ordem.

Uma condição necessária e suficiente para que um sistema $X^{\prime}=A X$ seja equivalente a uma equação de diferenças de $m$-ésima ordem é que $A$ seja uma matriz companheira. O próximo teorema mostra algumas condições equivalentes a $A$ ser uma matriz companheira (Veja LaSalle, [18]).

Proposição 3.9.1. São equivalentes:

1. A é uma matriz companheira.

2. $\operatorname{dim}(\operatorname{Im}(A-\lambda I))=m-1$ para cada $\lambda \in \sigma(A)$.

3. A multiplicidade geométrica de cada autovalor de Aé 1 .

4. O polinômio característico de A é o polinômio minimal de A.

5. Existe um vetor $b$ tal que $b, A b, A^{2} b, \ldots, A^{m-1} b$ são linearmente independentes. 


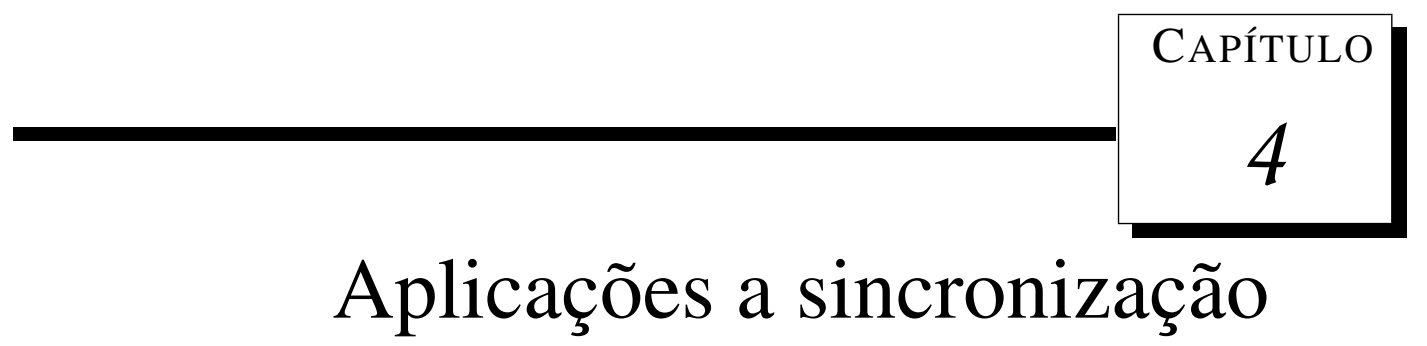

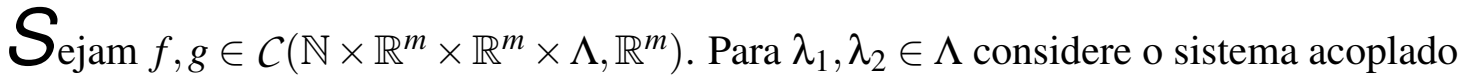

$$
\left\{\begin{array}{l}
x^{\prime}=f\left(n, x, y, \lambda_{1}\right) \\
y^{\prime}=g\left(n, x, y, \lambda_{2}\right)
\end{array}\right.
$$

Definição 4.0.2. Dizemos que o sistema 4.1 sincroniza globalmente (ou sisplemesmente sincroniza) se dado $\varepsilon>0$ existe $\delta>0$ tal que se $\left|\lambda_{2}-\lambda_{1}\right|<\delta$, então para todo $\left(x_{0}, y_{0}\right) \in$ $\mathbb{R}^{m} \times \mathbb{R}^{m}$ temos que $\limsup _{n \rightarrow \infty}\left|y\left(n, x, y, \lambda_{1}, \lambda_{2}\right)-x\left(n, x, y, \lambda_{1}, \lambda_{2}\right)\right|<\varepsilon$.

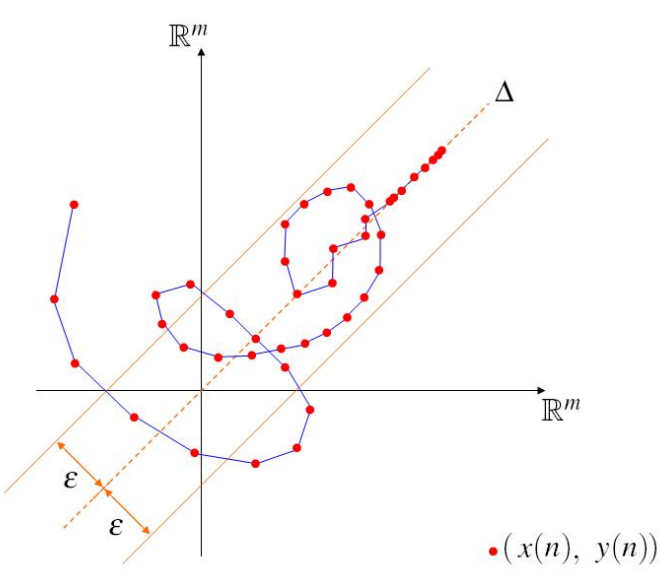

Figura 4.1: Ilustração da definição 4.0.2

\subsection{Aplicações ao sistema de Lorenz discreto}

Nessa seção apresentaremos algumas simulações relativas a sincronização do sistema de Lorenz discreto Acoplado, dado pelas equações: 


$$
\left\{\begin{array}{l}
x(n+1)=x(n)+h[-a x(n)+a y(n)] \\
y(n+1)=y(n)+h\left[-y(n)-\frac{r}{4}\left(x(n)+\alpha_{n}\right)-\left(x(n)+\alpha_{n}\right) z(n)\right] \\
z(n+1)=z(n)+h\left[-b z(n)+\left(x(n)+\alpha_{n}\right) y(n)-\frac{5}{4} b r\right] \\
u(n+1)=u(n)+h[-a u(n)+a v(n)] \\
v(n+1)=v(n)+h\left[-v(n)-\frac{r}{4}\left(x(n)+\alpha_{n}\right)-\left(x(n)+\alpha_{n}\right) z(n)\right] \\
w(n+1)=w(n)+h\left[-b w(n)+\left(x(n)+\alpha_{n}\right) y(n)-\frac{5}{4} b r\right]
\end{array} .\right.
$$

Para simular esse sistema, como no exemplo 2.3.1, Tomaremos $a=10, r=28, b=\frac{8}{3} \mathrm{e}$ $\alpha_{n}=\alpha(n)=4 \cos (n)$. Da observação 1.4.2 segue que $\overline{\{\alpha(n), n \in \mathbb{N}\}}=[-4,4]$.

Utilizando MATLAB com a seqüência de comandos:

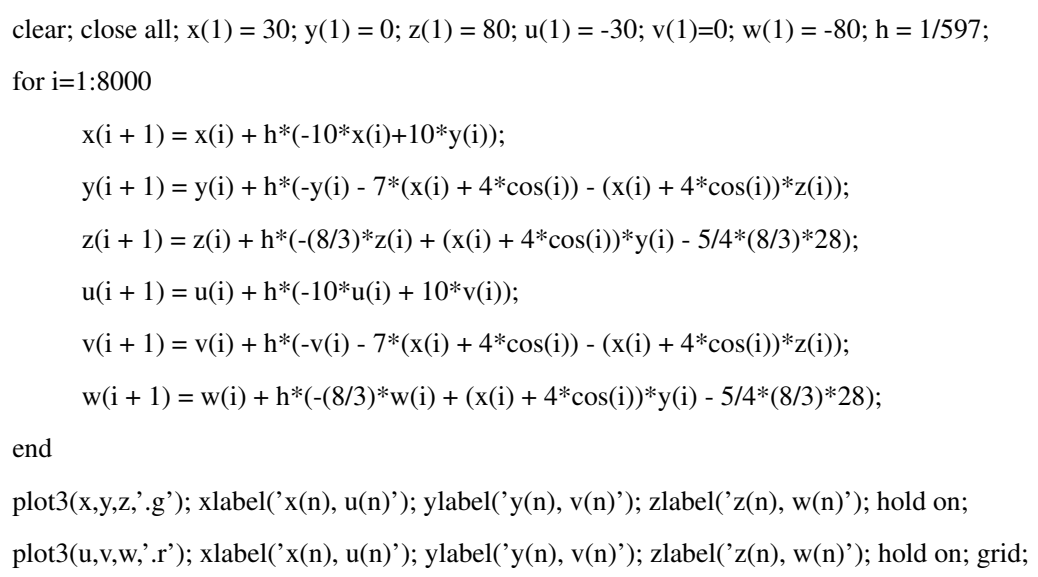

obtivemos as seguintes imagens, que mostram o comportamento assintótico de $(x(n), y(n), z(n))$ (verde) e $u(n), v(n), w(n))$ (vermelho), $n \in \mathbb{N}$ sob diferentes ângulos: 


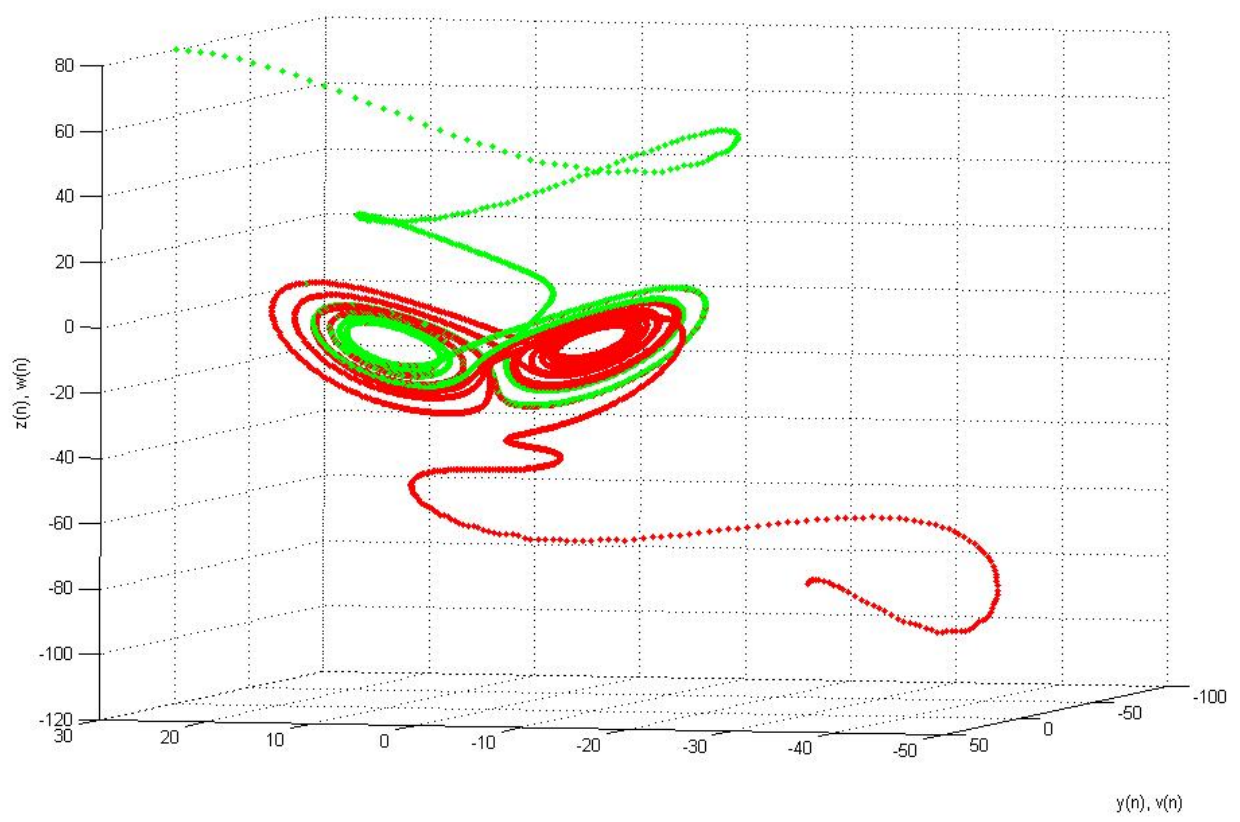

$x(n), u(n)$

Figura 4.2: Simulação de $(x(n), y(n), z(n))$ e $(u(n), v(n), w(n))$

Em seguida, utilizamos a seqüência de comandos:

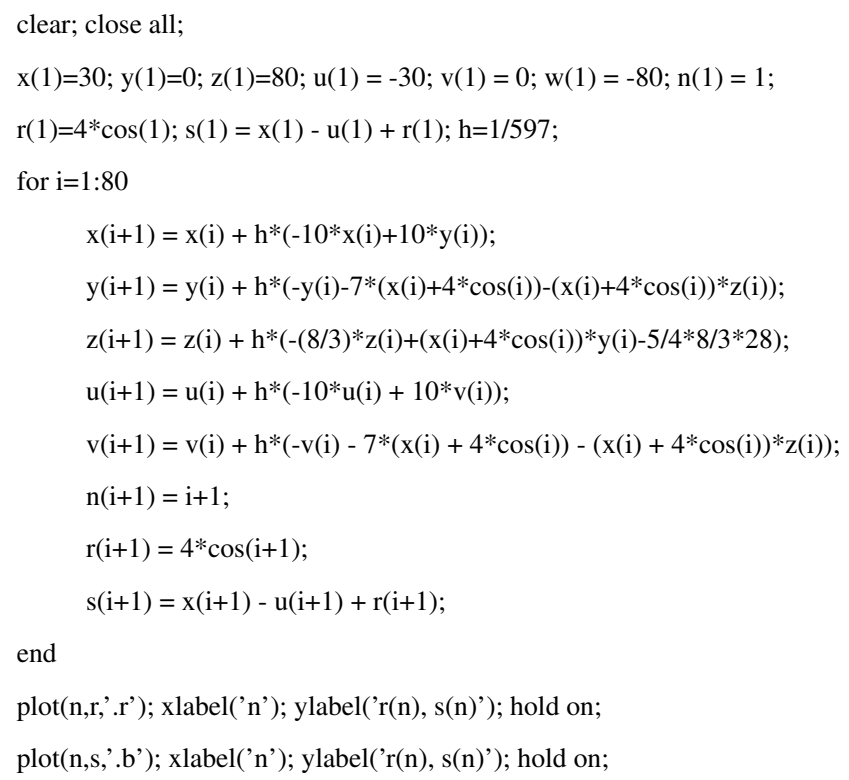

para plotar $\alpha(n)=4 \cos (n)$ (vermelho) e $\bar{\alpha}(n)=x(n)+\alpha(n)-u(n)$ (azul). $\quad \bar{\alpha}(n)$ é da decodificação (e recuperação) do sinal $\alpha(n)$. 


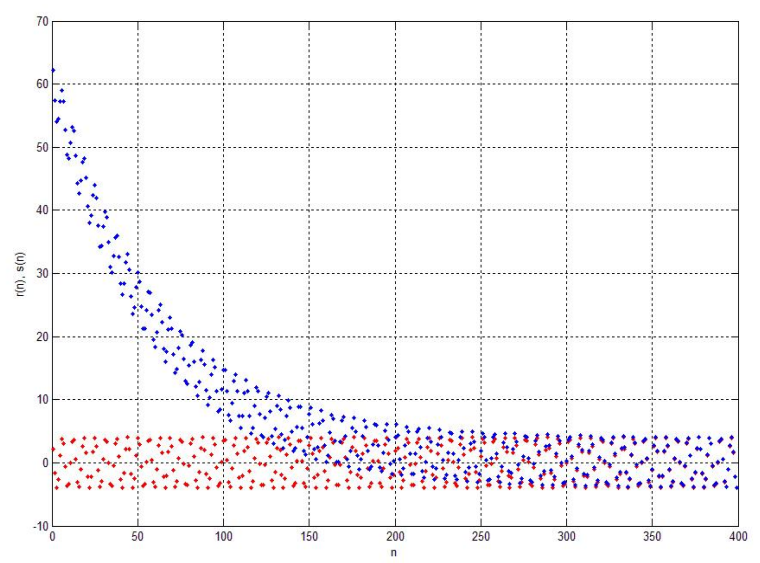

Figura 4.3:

Finalmente, simulamos a sincronização com a seqüência de comandos:

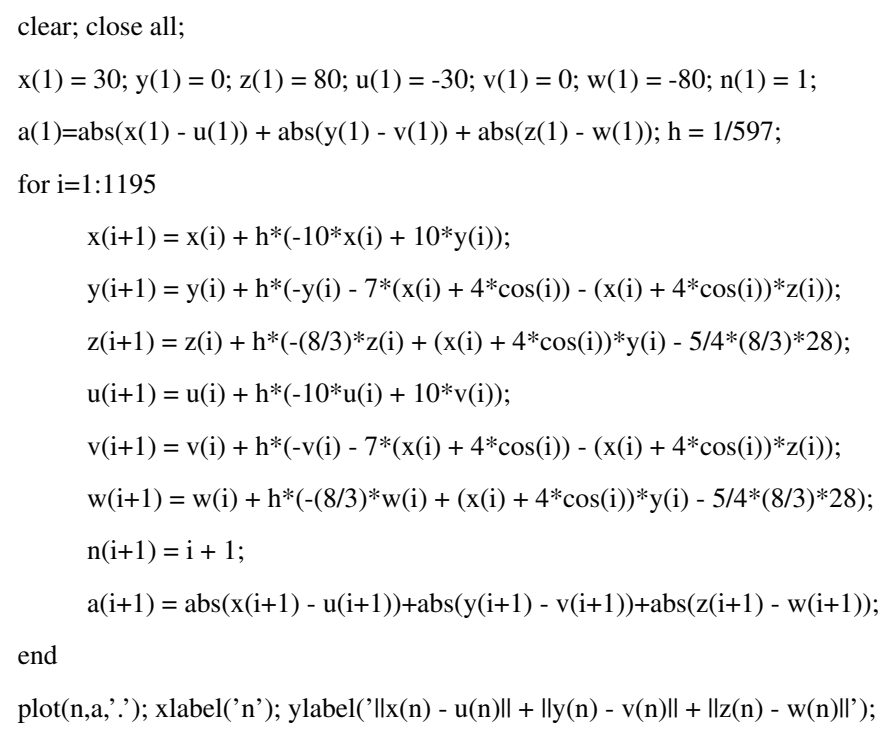


Obtivemos o gráfico:

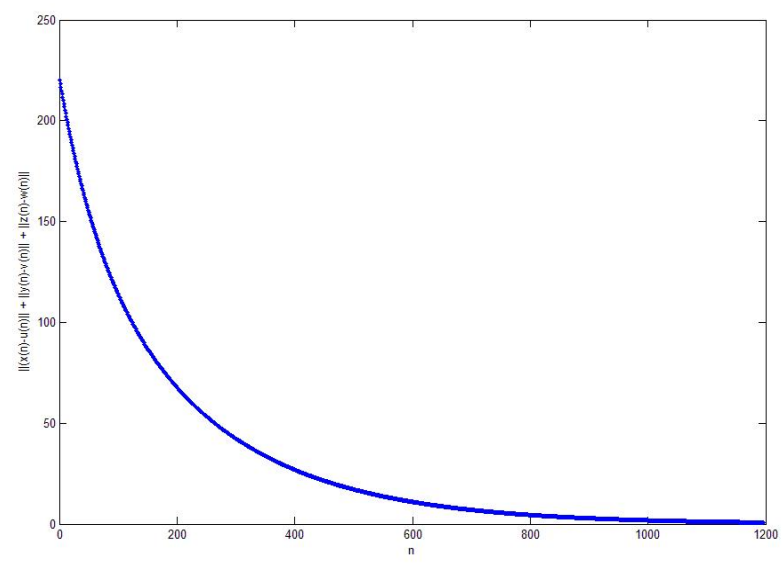

Figura 4.4:

Ou seja, computacionalmente obtivemos que $|x(n)-u(n)|+|y(n)-v(n)|+|z(n)-w(n)| \rightarrow$ 0 quando $n \rightarrow \infty$.

Como o lado esquerdo é uma soma de três parcelas positivas, temos que $|x(n)-u(n)| \rightarrow 0$, $|y(n)-v(n)| \rightarrow 0$ e $|z(n)-w(n)| \rightarrow 0$ quando $n \rightarrow \infty$.

Assim, $|(x(n), y(n), z(n))-(u(n), v(n), w(n))| \rightarrow 0$ quando $n \rightarrow \infty$, e como na definição 4.0.2, temos que esses sistemas sincronizam. 



\section{Referências Bibliográficas}

[1] AFRAIMOVICH, V. S.; VERICHEV, N. N.; RABINOVICH, M. I. Stochastic synchronization of oscilations in dissipative systems. Izv. Vyssh. Uchebn. Zaved. Rdiofiz. n 9, p. 1050-1060, 1986.

[2] AlBerto, L. F. C.; CALliERO, T. R; MARTINS A. C. P. An invariance principle for nonlinear discrete autonomous dynamical systems, vol 52, n 4, transactions on automatic control. (04/2007).

[3] ALBERTO, L. F. C. O princípio da invariância de Lassalle estendido aplicado ao estudo de coerência de geradores e à análise de estabilidade transitória multi"swing". 2000. Tese (doutorado em engenharia elétrica) - EESC, USP, São Carlos, 2000.

[4] BELLMAN, Richard. Introduction to matrix analysis. New York: McGRAW-HILL BOOK COMPANY, 1960.

[5] CALLIERO, Taís Ruoso. Um princípio de invariância para sistemas dinamicos discretos. 2005. Dissertação (mestrado em engenharia elétrica) - EESC, USP, São Carlos, 2005.

[6] CONWAY, John B. Functions of one complex variable I. 2. ed. EUA: Springer, 1978. (Graduate texts in mathematics, vol 11).

[7] FOLLAND, G. F. Real Analysis - modern techniques and their applications. New York: John Wiley and Sons, 1984.

[8] ^ GABRIEL FILHO, L. R. A. Comportamento assintótico de sistemas não lineares discretos. 2004. Dissertação (mestrado em matemática) - ICMC, USP, São Carlos, 2004.

[9] GARCIA, Arnaldo; LEQUAIN, Y. Elementos de álgebra, Rio de Janeiro: IMPA, 2001. (Projeto Euclides).

[10] HAHN, Wolfgang. Stability of motion. Nova York: Springer, 1967. 
[11] $\star$ HALE, Jack. K. Asymptotic Behavior of dissipative sistems. EUA: Americam Mathematical Society, 1988. (MATHEMATICAL SURVEYS AND MONOGRAPHS).

[12] _ Ordinary diferential equations. EUA: John Wiley \& Sons, 1969. (pure and applied mathematics).

[13] HARTMAN, P. Ordinary Differential Equations. J.Wiley, 1964.

[14] HENRICI, P. Applied and computational complex analysis, Vol 1. New York: Wiley, 1998.

[15] HIRSCH, Morris W.; SMALE, Stephen; DEVANEY, Robert L. Differential Equations, Dynamical Systems \& An Introduction to Chaos. 2. ed. EUA: Elsevier academic press, 2004. (pure and applied mathematics, vol 60).

[16] HOFFMAN, Kenneth; KUNZE, Ray. Álgebra Linear. tradução de Adalberto Panobianco Bergamasco, São Paulo: EDUSP, 1970.

[17] KATOK, Anatole; HASSELBLATT, Boris. Introduction to the modern theory of dynamical systems. 1995. (Encyclopedia of mathematics and its aplications).

[18] $\star$ LASALLE, Joseph P. The Stability and Control of Discrete Processes. Springer Verlag, 1986. (Applied Mathematical Sciences).

[19] $\star \ldots$. The Stability of Dynamical Systems. Springer - Verlag, 1986. (Applied Mathematical Sciences).

[20] LIMA, Elon Lages. Algebra Linear. Rio de Janeiro: IMPA, 1998. (Coleção Matematica universitária).

[21] _. Análise no espaço $\mathbb{R}^{n}$. Rio de Janeiro: IMPA, 2004. (Coleção Matematica universitária).

[22] _ Curso de análise volume. 1. 11. ed. Rio de Janeiro: IMPA, 2004. (Projeto Euclides).

[23] Curso de análise volume. 2. 8. ed. Rio de Janeiro: IMPA, 2005. (Projeto Euclides). . Espaços métricos. Rio de Janeiro: IMPA, . (Coleção Matematica universitária).

[25] LORENZ, E. N. Irregularity: a fundamental propertie of the atmosphere, Tellus, vol. 36A, p 98 - 110, 1984. 
[26] MUNKRES, James R. Topology, a first course. 2. ed. EUA: Prentice Hall, 2000.

[27] OTT, E.; SAUER, T.; YORKE, J. A. Coping with chaos: analysis of chaotic data and the exploitation of chaotic systems. EUA: John Wiley \& Sons, 1994.

[28] PALIS JUNIOR. J.; MELO. W. Introdução aos sistemas dinâmicos. Rio de Janeiro: IMPA, 1977. (Projeto Euclides).

[29] ROBINSON, Clark. DYNAMICAL SYSTEMS Stability, Symbolic Dynamics, and Chaos. 2. ed. Florida: CRC Pres LLC, 1998.

[30] RODRIGUES, H. M.; GAMEIRO, Marcio F. Aplications of Robust Synchronization to Comunication Systems, vol 79, p. 21-45 Aplicable Analysis.

[31] ^ RODRIGUES, H. M.; WU, Jianhong; GABRIEL FILHO, L. R. A. Uniform Dissipativeness and Robust Syncrhonization of Parametrized Discrete Systems: Location of the Atractor, vol 79, p. 21-45 Aplicable Analysis.

[32] RODRIGUES, H. M.; ALBERTO L. F. C., BRETAS N. G. On the invariance principle: Generalizations and Applications to Synchronizations, Journal of Diferential Equations 169, 228-254 (2000)

[33] RUDIN, Walter. Principles of mathematical analysis. New York: MacGraw-Hill, 1976.

[34] SOTOMAYOR, Jorge. Licões de equacões diferenciais ordinárias. Rio de Janeiro: IMPA, 1979. (Projeto Euclides). 


\section{Índice Remissivo}

$A_{\rho}, 24$

$B_{\rho}, 24$

$J(\alpha, \beta), 44$

$J(\lambda), 45$

$M_{ \pm}, 49$

$\operatorname{Per}(T), 10$

$\operatorname{Per}(T, k), 10$

$\Delta\left(\lambda_{1}, \ldots, \lambda_{m}\right), 55$

$\Lambda, 24$

$\Omega(H), 15$

П, 6

$\dot{V}(x), 20$

$\dot{V}(x, n, \lambda), 24$

$\dot{x}, 5$

$\gamma(x), 8$

$\gamma^{+}(x), 8$

$\gamma^{-}(x), 8$

$\mathbb{S}^{1}, 15$

C, 24

$\omega(x), 12$

$\rho(x, S), 15$

$\sigma(A), 43$

$\operatorname{diag}\left\{\lambda_{1}, \ldots, \lambda_{j}\right\}, 44$

$o(x), 52$

$r(A), 43$

$x^{\prime}, 5$

Atrator local, 19

Autovalores, 43

Conjunto

$\omega$-limite, 12

estável, 19 globalmente assint. estável, 19

instável, 19

invariante, 6

invariantemente conexo, 9

localmente assint. estável, 19

localmente atrativo, 19

negativamente invariante, 6

positivamente invariante, 6

uniformemente assint. estável, 19

Critério

de Schur-Cohn, 49

de Silvester, 50

Derivada discreta, 20

Determinante de Vandermonde, 55

Equação

de diferenças, 5, 53

Espectro, 43

Fórmula da variação das constantes, 55

Forma Canônica de Jordan, 45

Função

de Liapunov, 20, 49

de Liapunov generalizada, 24

definida positiva, 20

$\mathrm{o}(\mathrm{x}), 52$

Indice

de uma matriz nilpotente, 44

Lorenz, 28, 57

Matriz

companheira, 56

companheira principal, 54 
definida positiva, 49

estável, 48

internos de uma, 48

Menores principais de uma, 49

Multiplicidade

algébrica, 43

geométrica, 43

Norma de uma matriz, 43

Orbita

completa, 8

negativa, 8

periódica, 10

positiva, 8

Período, 10

Polinômio característico, 43, 54

ponto de equilíbrio

assintoticamente estável, 20

estável, 20

instável, 20

Ponto fixo, 10

Ponto periódico, 10

Princípio

da superposição, 55

de invariância, 21

Problema de valor inicial, 5

Prolongamento, 8

maximal, 8

Raio espectral, 43

Sincronismo, 57

\section{Sistema}

de equações de diferenças, 43

dinâmico, 6

semidinâmico, 6

Solução

periódica, 10
Teorema

da decomposição polar, 44

de estabilidade assintótica, 22

de estabilidade de Liapunov, 20

de Holmes, 48

do Ponto fixo de Banach, 12

do ponto fixo de Brower, 12 